\title{
WestVirginiaUniversity
}

THE RESEARCH REPOSITORY @ WVU

Graduate Theses, Dissertations, and Problem Reports

2020

\section{Asthma-COPD Overlap: Economic Burden and Medication Adherence}

\author{
Mona Nili \\ mn0044@mix.wvu.edu
}

Follow this and additional works at: https://researchrepository.wvu.edu/etd

Part of the Geriatrics Commons, Health Services Research Commons, Pharmacy Administration, Policy and Regulation Commons, and the Pulmonology Commons

\section{Recommended Citation}

Nili, Mona, "Asthma-COPD Overlap: Economic Burden and Medication Adherence" (2020). Graduate Theses, Dissertations, and Problem Reports. 7680.

https://researchrepository.wvu.edu/etd/7680

This Dissertation is protected by copyright and/or related rights. It has been brought to you by the The Research Repository @ WVU with permission from the rights-holder(s). You are free to use this Dissertation in any way that is permitted by the copyright and related rights legislation that applies to your use. For other uses you must obtain permission from the rights-holder(s) directly, unless additional rights are indicated by a Creative Commons license in the record and/ or on the work itself. This Dissertation has been accepted for inclusion in WVU Graduate Theses, Dissertations, and Problem Reports collection by an authorized administrator of The Research Repository @ WVU.

For more information, please contact researchrepository@mail.wvu.edu. 


\title{
Asthma-COPD Overlap: \\ Economic Burden and Medication Adherence
}

\author{
Mona Nili, MS, MBA, PharmD \\ Dissertation submitted \\ to the School of Pharmacy \\ at West Virginia University \\ in partial fulfillment of the requirements for the degree of \\ Doctor of Philosophy in \\ Health Services and Outcomes Research \\ Usha Sambamoorthi, Ph.D, Chair \\ Nilanjana Dwibedi, PhD, MBA, Co-Chair \\ Traci J. LeMasters, Ph.D. \\ Megan Adelman, PharmD \\ Suresh Madhavan, PhD, MBA \\ Department of Pharmaceutical Systems and Policy \\ Morgantown, West Virginia \\ 2020
}

Keywords: Asthma COPD Overlap, Healthcare Costs, Out-of-Pocket Burden, Cost-Related Medication Non-Adherence, Initial Maintenance Therapy, Medication Adherence

Copyright 2020 Mona Nili 


\section{ABSTRACT \\ Asthma-COPD Overlap: Economic Burden and Medication Adherence}

\section{Mona Nili}

In many older adults, asthma and chronic obstructive pulmonary disease (COPD) coexist as "Asthma-COPD overlap" (ACO). This dissertation pursued three Aims: 1) estimate the economic burden of ACO and its contributory factors; 2) evaluate the association of ACO to "cost-related medication nonadherence" (CRN); and 3) identify adherence trajectory patterns and examine the impact of initial maintenance therapies (IMT) type (inhaled corticosteroids monotherapy (ICS) vs. fixed-dose combination of ICS and long-acting beta agonist (ICS/LABA)) on adherence trajectory patterns among older adults Medicare Beneficiaries. The first and second Aims were cross-sectional studies by using Medicare Current Beneficiary Survey data. For the third Aim, we used a longitudinal retrospective cohort design, using $10 \%$ sample of Optum's Deidentified Clinformatics ${ }^{\circledR}$. In this study, older adults with ACO had nearly four times higher average total healthcare expenditures compared to those no-asthma no-COPD (NANC). The higher number of medications and fragmented care were the leading contributors of the economic burden. Moreover, compared to older adults with NANC, those with ACO were more likely to report any CRN. This association was highly dependent on number of medications. In addition, we identified four medication adherence trajectories to IMT among older adults with ACO. Those who using ICS/LABA were less likely to have "persistent low adherence" (Adjusted Odds Ratio=0.38). In summary, these findings highlight the need to reduce the economic burden and improve medication adherence among older adults with ACO. Additionally, using ICS/LABA for IMT may improve adherence to IMT among older adults with ACO. 


\section{DEDICATION}

I dedicate this dissertation to my husband, M. Ray Arvand, Esq., and my mentor, Dr. Usha

Sambamoorthi, who has always been extremely supportive and has set the cornerstone of my continued success in my career. 
First and foremost, I would like to express the deepest appreciation to my mentor Dr. Usha Sambamoorthi for her relentless guidance, support, and encouragement to pursue my dissertation idea. I am truly blessed to have a mentor like you. You are one of the most intelligent and humble people that I know. You believed in me those moments when I did not believe in myself. Without your constant oversight, feedback, and guidance, I would not have been able to complete this dissertation.

I would also like to thank my committee members, Dr. Nilanjana Dwibedi, Dr. Traci LeMasters, Dr. Megan Adelman, and Dr. Suresh Madhavan, for serving as my committee members. I would like to thank Dr. Dwibedi for accepting all my last-minute requests and providing valuable feedbacks. I am grateful to Dr. LeMasters for providing timely feedback and for helping me improve my writing skills. Dr. Adleman: thank you for your prompt feedback, motivational emails and suggestions on clinical aspects of my dissertation. Dean Madhavan: I cannot thank you enough for all the numerous opportunities that you have provided to me over the years; you have truly played a critical role in grooming me as a researcher and a critical thinker.

I am extremely thankful to West Virginia University, and all the faculty (Dr. Scott, Dr. Kelly, Dr. Kamal (the new Chair of the Department) and staff (Sherri, Deborah, Angie) and students of the PSP department for providing me the necessary support to succeed. I also want to acknowledge my wonderful cohort friends (Nazneen, Rudi, and Ikram) for being my biggest cheerleaders, for working through days and nights on projects with me before deadlines, and for giving me wonderful memories that I will always cherish. 
Last but not the least, I would also like to thank my husband. I cannot thank you enough for your patience, 16 hours driving every two weeks from NYC to Morgantown, and all the wonderful BBQs to uplift my mood during stressful times. You always made me believe that I can do this. Thank you so much. I would like to acknowledge my family for their unconditional love and support which helped me throughout my life. I am extremely thankful to my mom for all the sacrifices she made for me to make me what I am today. 


\section{Table of Contents}

List of Tables viii

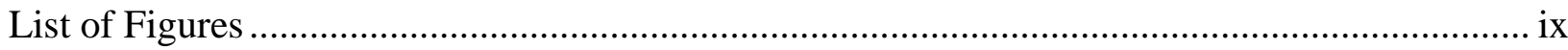

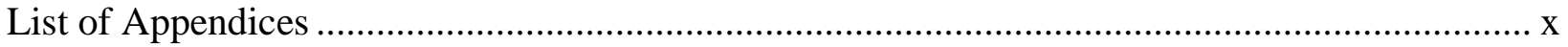

List of Abbreviations ......................................................................................................

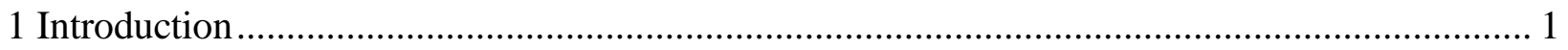

1.1 Background and Significance ..................................................................... 1

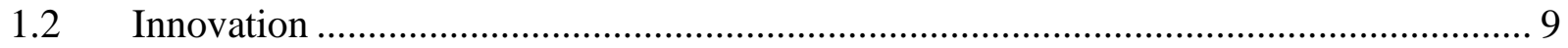

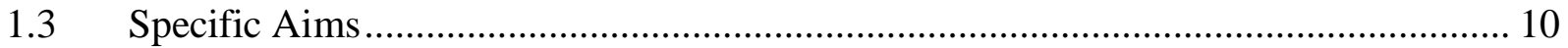

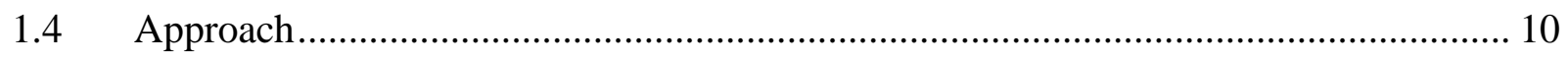

2 Economic Burden of Asthma- Chronic Obstructive Pulmonary Disease Overlap (ACO) among

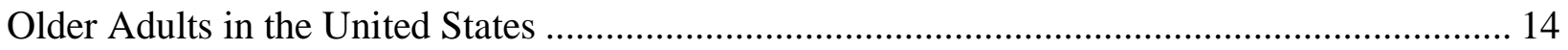

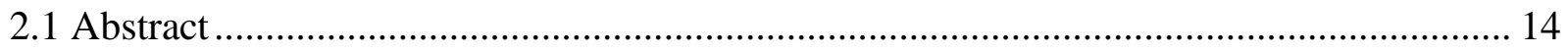

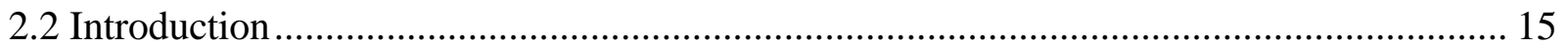

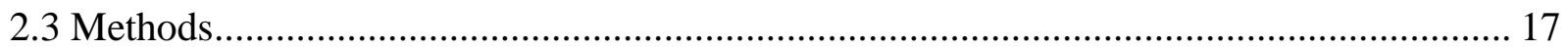

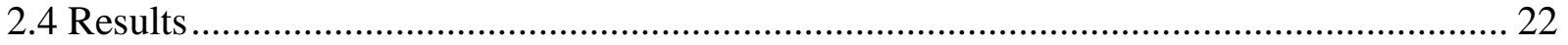

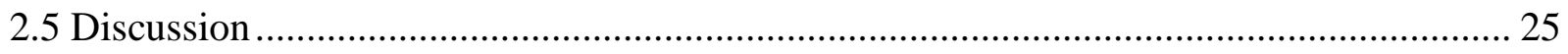

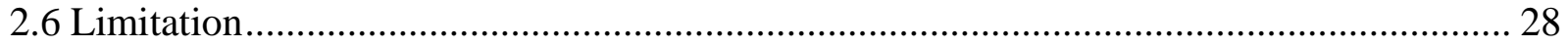

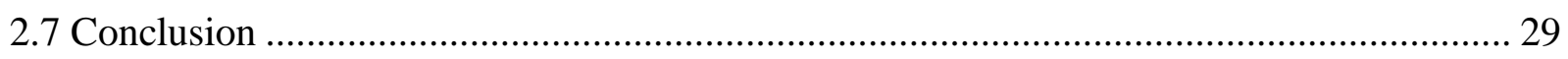

3 Asthma-Chronic Obstructive Pulmonary Disease Overlap and Cost-Related Medication

Nonadherence among Older Adults in the United States ................................................... 34

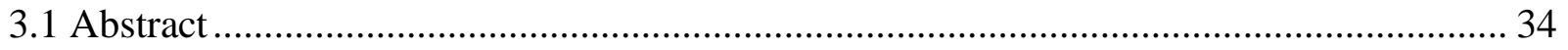

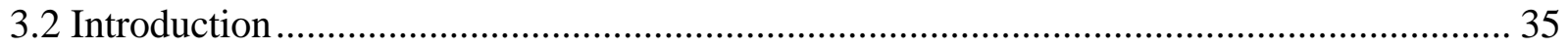

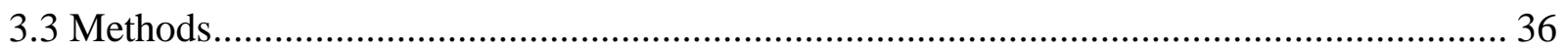




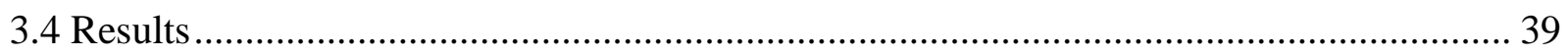

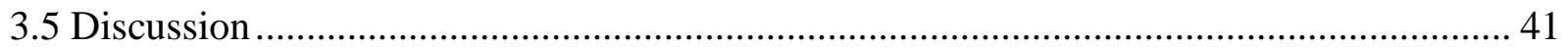

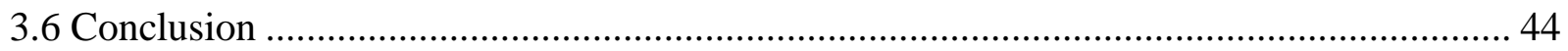

4 Impact of Initial Maintenance Therapy Type on Medication Adherence Trajectories among

Older Adults with Asthma and COPD Overlap ...................................................................... 50

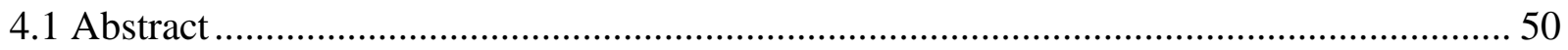

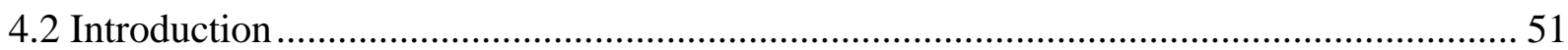

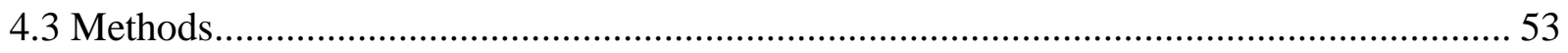

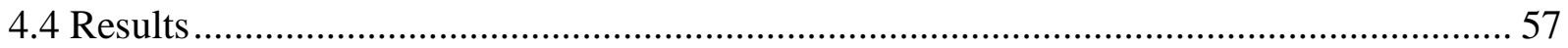

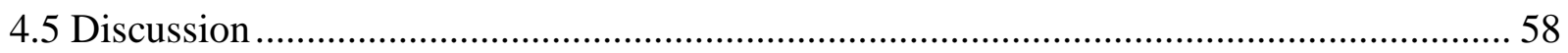

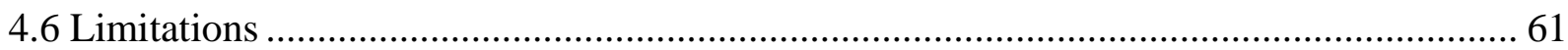

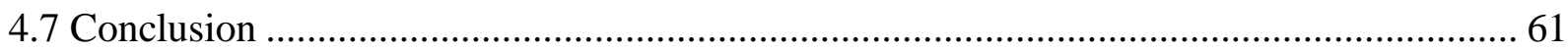

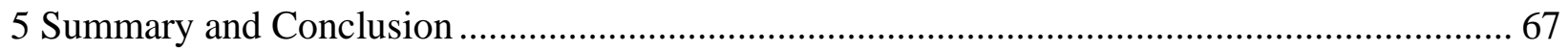

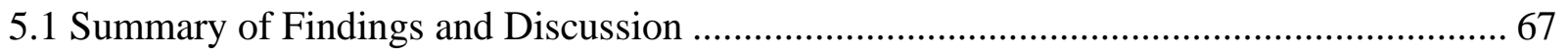

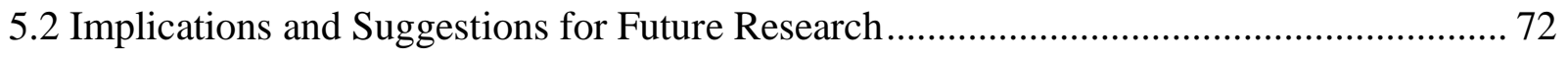

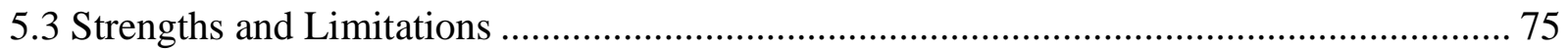

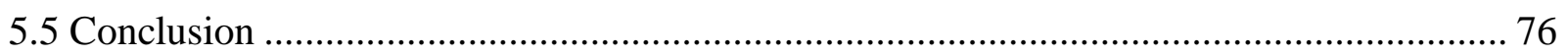

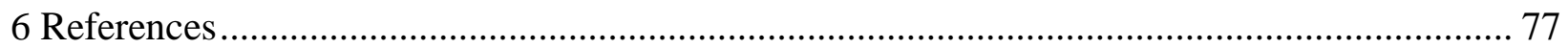

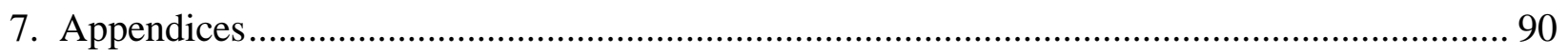




\section{List of Tables}

Table 2.1 Unweighted N and Weighted Percentages of Characteristics by Asthma /COPD Categories (Column \%) among Older (age > 65 years), Medicare Beneficiaries Medicare Current

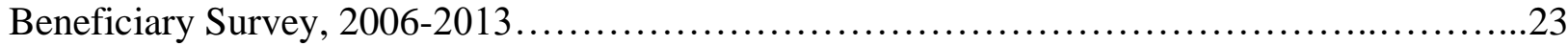

Table 2.2 Post-Regression Decomposition Analyses among Older (age > 65 years) Medicare Beneficiaries, Medicare Current Beneficiary Survey, 2006-2013.

Table 3.1 Unweighted $\mathrm{N}$ and Weighted Percentages of Characteristics by Asthma and Chronic Obstructive Pulmonary Disease (COPD) Status (Column \%) among Older (age > 65 years) Medicare Beneficiaries, Medicare Current Beneficiary Survey, 2006-2013.

Table 3.2 Unadjusted Odds Ratios (UOR), Adjusted Odds Ratios (AOR) and 95\% Confidence Intervals from Separate Logistic Regressions on Any Cost-related Medication Nonadherence among Older (age > 65 years) Medicare Beneficiaries, Medicare Current Beneficiary Survey, 2006-2013.

Table 3.3 Unadjusted Odds Ratios (UOR), Adjusted Odds Ratios (AOR) and 95\% Confidence Intervals from Separate Logistic Regressions on Specific Forms of CRN Older (age > 65 years) Medicare Beneficiaries, Medicare Current Beneficiary Survey, 2006-2013 ...................48

Table 4.1 Characteristics of Older Adults with Asthma-COPD Overlap by Initial Maintenance Therapy Options Inverse Probability Treatment Weighting, Optum Clinformatics 10\% Sample, 2007-2017

Table 4.2 Characteristics of Older Adults with Asthma-COPD Overlap by Medication Adherence Trajectories Initiated on Maintenance Therapy Optum Clinformatics 10\% Sample, 2007-2017.

Table 4.3 Unadjusted Odds Ratios (UOR), Adjusted Odds Ratios (AOR) and 95\% Confidence Intervals from Multinomial Logistic Regressions on Medication Adherence Trajectory Groups with Persistent High Adherence as Reference Group, Inverse Probability Treatment Weighting among Older Medicare Beneficiaries with ACO and Initiated on Maintenance Therapy, Optum Clinformatics 10\% Sample, 2007-2017. 


\section{List of Figures}

Figure 1.1 Conceptual Framework for Factors Associated with Healthcare Expenditures, Adapted from Anderson's Model).............................................................11

Figure 2.1 Total Healthcare Expenditures and High Out-of-Pocket Burden by Asthma/ COPD Categories among Older (age > 65 years) Medicare Beneficiaries, Medicare Current Beneficiary

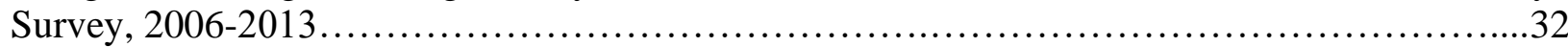

Figure 3.1 Prevalence of any CRN and Specific Types of CRN by Asthma-COPD Status (i.e., Asthma-COPD Overlap, Asthma-Only, COPD-Only, Neither Asthma nor COPD) among Older (age > 65 years) Medicare Beneficiaries Medicare Current Beneficiary Survey, 2006-2013 ......49

Figure 4.1 Trajectories of Adherence to Initial Maintenance Therapy among Older ( $>65$ years old) Adults with Asthma-COPD Overlap, Optum Clinformatics 10\% Sample, 2007-2017........66 


\section{List of Appendices}

Appendix 7.1 Attrition Table of Individuals Included in the Analysis Based on Inclusion and

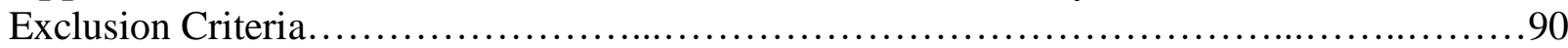

Appendix 7.2 Diagnosis Codes for Asthma and COPD .................................... 91

Appendix 7.3 Characteristics of Elderly Community-Dwelling Fee-for-Service Medicare Beneficiaries.

Appendix 7.4 Mean Annual Adjusted and Unadjusted Expenditure among Older (age > 65 years) Medicare Beneficiaries Medicare Current Beneficiary Survey, 2006-2013. ...

Appendix 7.5 Unadjusted Odds Ratios (UOR), Adjusted Odds Ratios (AOR) and 95\% Confidence Intervals from Separate Logistic Regressions on High Out-of-Pocket Burden among Older (age > 65 years) Medicare Beneficiaries Medicare Current Beneficiary Survey, 20062013.

Appendix 7.6 Post-Regression Decomposition Analyses among Older (age > 65 years) Medicare Beneficiaries Medicare Current Beneficiary Survey, 2006-2013.

Appendix 7.7 Attrition Table of Individuals Included in the Analysis Based on Inclusion and Exclusion Criteria

Appendix 7.8 Characteristics of Elderly Community-Dwelling Fee-for-Service Medicare Beneficiaries .97

Appendix 7.9 Attrition Table of Individuals Included in the Analysis Based on Inclusion and Exclusion Criteria.

Appendix 7.10 Adjusted Odds Ratios (AOR) and 95\% Confidence Intervals from Generalized Estimating Equation (GEE) on Adherence among older adults with ACO Who Newly Initiated Maintenance Therapy, Optum Clinformatics 10\% Sample, 2008-2016

Appendix 7.11 Characteristics of Older Adults with Asthma-COPD Overlap by Initial Maintenance Therapy Options before Using Inverse Probability Treatment Weighting, Optum Clinformatics 10\% Sample, 2007-2017. 


\section{List of Abbreviations}

ACO

AOR

CI

COPD

CRN

FFS

GBTM

GEE

GLM

HMO

ICD-9

ICD-10

IPTW

OOB

OOP

OR

SD

SE

SIG

UOR

US
Asthma COPD Overlap

Adjusted odds ratio

Confidence interval

Chronic Obstructive Pulmonary Disease

Cost-Related Medication Non-Adherence

Fee-for-service

Group-Based Trajectory Modeling

Generalized Estimating Equations

Generalized Linear Model

Health Maintenance Organization

International Classification of Diseases, Ninth Revision, Clinical Modification

International Classification of Diseases, Tenth Revision, Clinical

Modification

Inverse Probability of Treatment Weighting

Out of Bag

Out-of-pocket

Odds Ratio

Standard deviation

Standard error

Significance

Unadjusted Odds Ratio

United States 


\section{CHAPTER 1}

\section{Introduction}

\subsection{Background and Significance Epidemiology of Major Depressive Disorder}

Asthma and Chronic Obstructive Pulmonary Disease (COPD) are the two most common respiratory diseases in the world. Combined, more than 435 million adults suffer from asthma or COPD. 1,2. The economic burden of asthma (\$82 billion in 20153) and COPD (\$49 billion projected in 20204) in the United States are very high. Clinicians can easily make these distinctions. Asthma usually develops in childhood, has classic symptoms of chest tightness, wheezing, coughing, and dyspnea, and is more likely to be associated with atopic disorders. COPD usually manifests later in life with dyspnea and productive cough and more likely be associated with environmental factors such as tobacco smoking.

\section{Definition of Asthma-Chronic Obstructive Pulmonary Disease Overlap (ACO)}

In 2009, Gibson and Simpson reported that about half of the older patients with obstructive airway disease have overlapping symptoms of both asthma and COPD. They called this the asthma-COPD “overlap syndrome” 5. In 2013, "asthma-COPD overlap syndrome” (AOCS) was used to describe this condition which is still used in many studies6. However, recent studies have suggested that it is better to describe this condition as "asthma COPD overlap" (ACO) without using "syndrome" since there is no specific definition of syndrome for this condition7. Thus, in our study, we use the term ACO when asthma and COPD co-exist in the same individual.

\section{Prevalence of ACO}

In the general population, the estimated prevalence of ACO varies widely across studies based on the definitional criteria of ACO and time period. Using the National Health and 
Nutrition Examination Survey (NHANES)-III (1988-1994) data, Diaz-Guzman et al. estimated that the prevalence of ACO was 2.7\% . However, and estimated the prevalence of ACO was estimated at $0.96 \%$ in the period spanning 2007-2012. Prevalence of ACO among individuals with COPD or Asthma.

The prevalence of ACO among patients with either asthma or COPD is higher than the general population. According to the findings of a meta-analysis, the global prevalence of ACO among patients with asthma is $26.5 \%$ and among patients with COPD is $29.6 \% 9$ However, the prevalence of ACO can be as high as An estimated 50\% patients with asthma or COPD9. Among patients with obstructive lung diseases, the prevalence of $\mathrm{ACO}$ is less than $10 \%$ among younger patients $(<50$ years) and more than $50 \%$ among older patients $(\geq 80$ years or older) 10 . Similar results on age distribution were found in the Italian population6.

\section{Disease Burden of ACO}

Disease burden of a condition is often measured with morbidity, health-related quality of life, mortality, healthcare utilization and costs. ACO patients experience substantial disease burden. In terms of mortality, ACO may be associated with a higher mortality compared with either asthma or COPD. In the NHANES-III cohort, Diaz-Guzman et al found that the hazard ratio (HR) for mortality in ACO is the highest (1.8), compared with COPD (1.4) and asthma (1.2)8. These findings contrast with few other studies where ACO have not increased risk of negative consequences. For example, a study of older adults ( $\geq 55$ years old) with progressive obstructive airway disease found that COPD patients had a worse prognosis compared to patients with $\mathrm{ACO}_{11}$. Bai et al also found that patients with $\mathrm{ACO}$ had higher likelihood of better prognoses and lower mortality compared to patients with COPD alone, though with a heightened exacerbation frequency. 
In a cohort of 17,088 COPD patients in Taiwan, ACO patients experienced almost twice as many acute respiratory events (i.e. acute respiratory failure, pneumonia, acute exacerbation, and cardiopulmonary arrest) than did the rest of the COPD cohort12. In the Latin American Project for the Investigation of Obstructive Lung Disease (PLATINO), patients with ACO had worse lung function, had more respiratory medications, experienced more exacerbations and hospitalizations and reported a worse perception of general health status than patients with only either asthma or COPD13.

The prevalence of co-existing health conditions is also higher in ACO compared to either asthma or COPD. A population survey in the US found that patients with ACO found that $90 \%$ of respondents had at least one other co-existing condition (compared with $71 \%$ in asthma and $84 \%$ in COPD) 14 . When analyzed by type of eight chronic comorbid conditions (e.g. cardiovascular diseases, diabetes, and depression), the prevalence of all eight conditions were higher in ACO compared to asthma, and six out of eight conditions were more prevalent in ACO compared to COPD.

\section{Economic burden of ACO}

The high morbidity burden experienced by patients with ACO may also translate into high healthcare utilization and high healthcare costs. Using the 2009 Korean National Health Insurance database on 185,147 COPD patients, Rhee et al found that those with ACO had a higher frequency of emergency department visits, hospitalization and intensive care unit visits than patients with only COPD15. In this study, healthcare utilization and costs were significantly higher for both outpatient and inpatient care for ACO patients compared to those with only COPD or only asthma15. In a retrospective cohort study in Taiwan, patients with ACO had the higher medication costs, and experienced more respiratory-related hospital visits compared to 
patients with asthma or COPD 16. In Canada, a retrospective cohort study reported that costs of medications and outpatient services were higher in ACO patients, but costs of hospitalizations were lower compared to patients with COPD. In this study, costs of non-respiratory conditions were lower in ACO, mainly due to lower costs of cardiovascular diseases.

Most of the studies on economic burden of ACO has been conducted mainly outside the United States (US). There have been only two studies in the US on the economic burden of ACO. A retrospective cohort analysis of commercial claims in the US, both asthma-related and all-cause health care expenditures were twice as high for patients with ACO compared to those with only asthma17. Among enrollees of Medicare Advantage Plan in 2004, Blanchett et al found that mean unadjusted respiratory-related health care expenditures were $\$ 7,240$ for the patients with $\mathrm{ACO}$ and $\$ 5,158$ for patients with COPD 18 . However, these studies were conducted a decade ago and the estimates are dated. Furthermore, the contributing factors to the excess cost of ACO remains unknown. The presence of comorbidities, more frequent exacerbations, and higher healthcare and medication utilization may be the major drivers of this excess cost.

\section{The Importance of Medication Use in ACO}

An important goal of ACO management is slowing the progression of the disease as there is no cure for ACO. Inhaler medications play an important role slowing the progression of the disease. For example, medication fluticasone furoate/vilanterole, as an ICS/LABA, has been shown to substantial improvement in lung functions 19. Therefore, adherence to medication is expected to be important among individuals with ACO. Although very limited evidence support the effect of adherence to inhaler medication and clinical outcomes, previous studies among patients with COPD and asthma support this20,21.

\section{Link between Medication Nonadherencee and Economic Burden}


Medication non-adherence is generally a source of considerable cost to the U.S. health care system22. Because medication adherence can accelerate the progression of the disease leading to hospitalizations, emergency room visits and other healthcare services. Osterberg and Blaschke estimated that medication non-adherence contributes to approximately $\$ 100$ billion in hospitalizations and \$2,000 per patient annually in excess physician visits.23 Among Medicare beneficiaries with COPD, Simoni-Wastila et al estimated that patients who were not adherent to their medications had $\$ 2,185$ higher costs compared with patients who adhered to their medication24. Specific to asthma and COPD, medication adherence to maintenance therapy is critical to slow the progression of disease. For example, a retrospective cohort study reported that poor adherence to initial maintenance therapy was associated with progression of disease among patients with moderate to severe asthma25.

\section{Medication Nonadherencee among Adults with ACO}

As there are no published studies on medication adherence among ACO patients, we speculate that ACO patients may be particularly vulnerable to medication non-adherence based on published literature focusing on either asthma or COPD or chronic respiratory conditions. As asthma and COPD are chronic conditions, patients with ACO are expected to use medications for asthma as well as COPD for the remainder of their lives. In general, medication adherence among patients with chronic pulmonary disease is lower compared to other chronic conditions, including rheumatologic conditions, HIV, cancer, and gastrointestinal disorders 26 In a systematic review with more than 2,000 subjects with asthma using ICS, medication adherence ranged from $47 \%$ to $57 \% 27$. Similarly, a study of 244 patients with COPD reported that adherence to ICS/LABA was less than 23\%28. Non-adherence with long-acting bronchodilators was found among $65 \%$ of 45,000 patients with COPD in Germany29. This number was $70 \%$ among 11,000 
COPD patients in the US Veterans Affairs database30. Poor adherence to maintenance therapy is particularly higher among the elderly population. In a retrospective cohort study on Medicare beneficiaries with COPD, the average monthly adherence to IMT was very low31. In this study, the highest medication adherence was in the second month (0.57) and decreased rapidly before plateauing at 0.35 by the seventh month 31 .

\section{Higher Vulnerability of Older Adults with ACO to Medication Non-Adherence}

Older adults with ACO may be at higher risk for medication non-adherence due to high medications costs32. Specifically, adherence to maintenance therapy may also be lower due to therapy-related factors such as medication type, ease of administration, ease of dosing 33 . While high cost of medications and medication-related factors can affect adherence among all patients with ACO, the older patients may be at a higher risk due to a unique set of circumstance (such as the high prevalence of comorbid conditions, low socio-economic status, and high rates of polypharmacy).

\section{Cost Related Medication Non-Adherence (CRN) among Older Adults with ACO}

The primary socioeconomic factors influencing medication non-adherence are access to prescription benefits and out-of-pocket expenses34. Without appropriate drug coverage, maintenance medications are cost prohibitive as generic maintenance medications have not been available in the United States35. In the US, most older patients are covered by Medicare insurance36. Although since 2006 Medicare Part D provided prescription drug coverage to increase access to prescription medications for the elderly population, most Medicare plans have drug coverage gaps (e.g. donut hole) that can create financial distress for the elderly patients37. High out-of-pocket contributions can increase CRN among elderly Medicare beneficiaries38. Moreover, formulary tiers make some of the most effective maintenance medications 
inaccessible35. Affected older patients often respond to the drug coverage gap by using costcoping behaviors (e.g. forgoing mortgage payments) and CRN behaviors (e.g. skipping some doses of medication for stretching medication possession time)39. Medicare beneficiaries with chronic health conditions have a higher risk of CRN behaviors.40-42 In 2006 Castaldi et al estimated that the prevalence of CRN is $31 \%$ among Medicare beneficiaries with chronic pulmonary diseases (e.g. asthma or COPD).32 They did not specify which chronic pulmonary diseases increases the risk of CRN. By considering the negative health outcomes of ACO, we patients with ACO may be at high risk of CRN.

\section{Treatment-Related Factors and Medication Non-Adherence}

Type and complexity of treatment are some of the many medication-related factors that may affect adherence to maintenance therapy among patients with asthma and COPD medications35. Most of the elderly patients are required to take multiple medications each day; many of these medications need more than one daily dose. ACO patients, who are already on complex regimens, adhering to complex regimen maintenance medications is challenging 43 and consequently may lead to medication non-adherence. Furthermore, maintenance medications use inhalers and older patients may face challenges in self-administering inhaler medications. Misperceptions about maintenance medications may also lead to medication nonadherence older patients44. For instance, fast relief by using short-acting bronchodilator can be perceived as a measure of potency. This can result in mistakenly prioritized short acting bronchodilators over maintenance medicines (e.g. ICS). Fears of addiction to ICS or developing a tolerance is also very common which can lead to poor adherence to maintenance medications 45. 


\section{Possible Impact of Medication Type on Medication Non-Adherence}

The joint guidelines of GINA and GOLD for treating ACO recommend ICS for IMT. The other IMT option for patients with ACO is ICS/LABA. The earliest use of ICS/LABA for IMT in treatment for ACO patients is supported by a study conducted by Gershon and colleagues 46 . In a case-control study, they demonstrated that newly prescribed ICS/LABA therapy, compared with LABA alone, was associated with a significantly lower risk of COPD hospitalization or death among ACO patients. Moreover, in a 12-week randomized open-label cross-over study, ACO patients experienced significant improvement in vital capacity after eight weeks ICSLABA treatment19. Prescribing ICS/LABA as IMT for ACO has two main advantages. First, ACO has both inflammatory and obstructive nature. Therefore, adding LABA to ICS can reduce obstruction. Second, using LABA is associated with a decreased risk of myocardial infarction, hospitalization, and death among patients with $\mathrm{ACO}_{47}$.

Although both ICS and ICS/LABA are possible options for IMT of ACO, their impact on adherence is unknown. A study of asthma patients reported poor medication adherence to IMT when ICS is in fixed-dose combination with LABA (ICS/LABA) compared to ICS monotherapy48. As medication adherence in general declines over time for respiratory and other conditions, there is a need to examine trajectories of medication adherence among ACO patients by types of IMT.

In summary, scientific studies of contributory factors to economic burden, cost-related mediation non-adherence and the impact of the type of maintenance therapy on adherence over time among ACO patients is non-existent. A deeper understanding of these issues is critical to providers as well as policy makers including CMS, patients, and providers. Contributory factors of the economic burden can be used by CMS in deriving risk-based cost estimates that are being 
used in value-based reimbursement models. The possible association of ACO to CRN for developing targeted intervention to reduce CRN. Our cost estimates can also be used to evaluate cost-effectiveness of interventions of care for ACO patients. By examining the possible impact of medication type (ICS/LABA vs ICS) on medication adherence, our study findings can provide real-world evidence on adherence patterns.

\subsection{Innovation}

a) The present study will be a "series of firsts." The study findings will fill a knowledge gap in the health outcomes of ACO in a nationally representative sample of the community dwelling Medicare beneficiaries in the US. For example, this is the first study to evaluate contributing factors to cost burden, $\mathrm{CRN}$ and long-term Medicare adherence among ACO patients.

b) Leveraging data from nationally representative surveys and medical claims data, we are able to have the "best of both worlds". The unique feature of MCBS-linked Medicare is the linkage between survey data and Medicare claims. In the survey component of the MCBS, the self-reported information not available in insurance claims databases (e.g. functional limitations, health status, smoking habit, and others). The Medicare claims component provides fee-for-service claims for both Part A and B. These claims include information on treatment, diagnosis, health services utilization, and Medicare payments49.

c) Generate new knowledge that may be used to improve medication adherence by evaluating modifiable factors such as regimen complexity and the out-of-pocket cost burden on CRN.

d) The application of advanced econometric and novel methods such as "group-based trajectory modeling" (GBTM). GBTM has been extensively applied in psychology, and 
criminology, but very few studies evaluated medication adherence using GBTM.

Longitudinal evaluation of adherence using GBTM can yield more useful classifications compared to traditional methods such as proportion of days of coverage (PDC).

\subsection{Specific Aims}

AIM 1: Estimate the excess economic burden of ACO (direct healthcare expenditures) and its contributory factors among older Medicare beneficiaries using post-regression decomposition techniques.

Hypothesis: A majority of the excess economic burden of ACO can be explained by medication non-adherence, fragmented care, and the presence of other comorbidities.

AIM 2: Evaluate the adjusted association of ACO to CRN among older Medicare beneficiaries.

Hypothesis: Older adults with ACO will be more likely to report $C R N$ and this can be explained by medication regimen complexity and a high out-of-pocket (OOP) cost burden.

AIM 3: Examine the impact of medication type (ICS vs ICS/LABA) on adherence over time among ACO patients who initiated maintenance therapy.

Hypothesis: Adults with ACO who use ICS/LABA have poorer adherence to maintenance therapy over time.

\subsection{Approach}

\section{Conceptual Framework}

We adopted features from the Anderson Health behavior modelso to study all aims in this study (Figure 1). This model has been used in many studies with different health conditions to study health care expenditures. According to this model, health care expenditure is determined 
by five factors: (1) predisposing factors, (2) enabling factors, (3) need factors, (4) personal health practices, (5) external factors. Predisposing factors are those reflecting an individual's propensity to use health services. These include factors like age, race/ethnicity, etc. Enabling factors are those that facilitate access to health care services. Examples of enabling factors include education status, health insurance coverage. etc. Need factors are potential needs for health services use, for example presence of comorbidities. Personal health practices factors reflect an individual's lifestyle. These include factors such as smoking habits and obesity. External factors are those related to environmental factors (e.g. region of residency).

Figure 1.1

Conceptual Framework for Factors Associated with Healthcare Expenditures Adapted from Anderson's Model

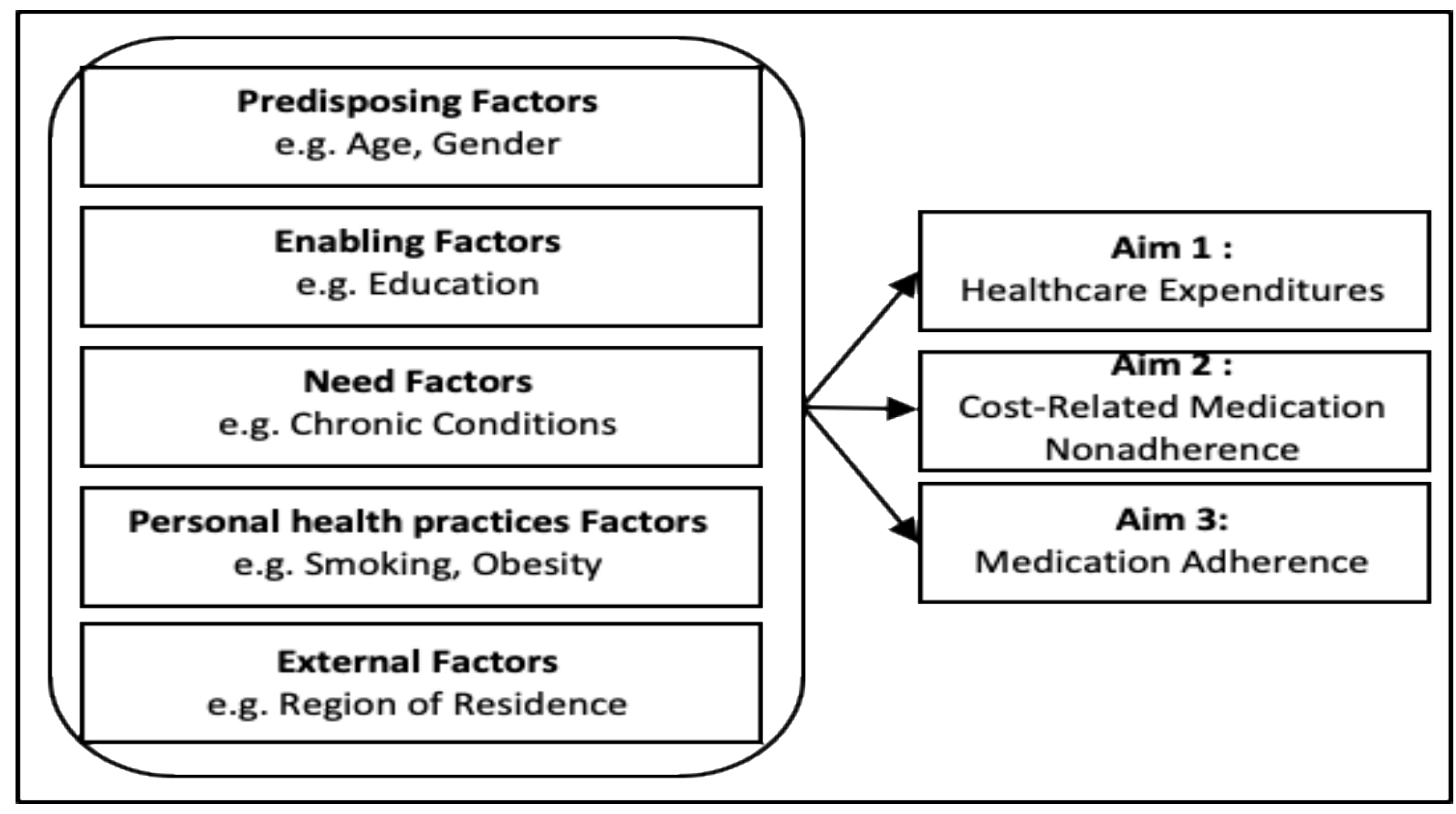

\section{Data Sources}

We will leverage multiple data sources to accomplish our research Aims. For Aims 1 and 2, we will use Medicare Current Beneficiary Survey (MCBS) linked to Medicare claims. For Aim 3, we will use the $10 \%$ random sample of Optum Clinformatics Data Mart®. 


\section{MCBS}

The MCBS is a continuous, multipurpose survey of a nationally representative sample of the Medicare population, conducted by CMS. The MCBS is conducted on a representative sample of Medicare beneficiaries. The survey is repeated annually for three years51. All participants are interviewed in person or with computer-assisted personal interview technology. From 1991 to 2013, the MCBS released two data files, the Access to Care (AC) and the Cost and Use (CU) annually, although the samples in both files share substantial overlap. The AC file can be linked to the CU file. Each year of the MCBS may be used for cross-sectional analyses or for conducting longitudinal analyses. We will use following files for Aims 1 and 2:

MCBS - Access to Care (AC) Files: The AC files contain information on beneficiaries' sociodemographics, self-reported health status and conditions, health insurance coverage, medication adherence, and cost-related medication adherence. The AC files represent the "always enrolled," Medicare beneficiaries. This file does not include survey-reported use of health care services and costs.

MCBS - Cost and Use (CU) Files: The CU file is designed to provide a complete user file, and it uses an "ever enrolled" population. The "ever enrolled" population includes health care services and costs for all Medicare beneficiaries who joined the program during the year and those who died during the year. The CU file includes survey reports for services not included in Medicare billing files (e.g. prescription drugs). Moreover, for Medicare covered services, the accuracy of service types, payments, and sources of payment has been improved by using an extensive operation to match and reconcile survey reports and Medicare bills.

MCBS-Medicare Claims Files: The CU and AC files can be linked to Medicare Fee-forService claims data. This will provide a unique opportunity for researchers to obtain a 
comprehensive picture of health services received, sources of payment, amounts paid, and including payments which are not covered by Medicare49. Medicare claims files include all Part A and B claims files. However, there is no separate file for Part D events. Information about prescription medications are is available in Prescribed Medicine Event (PME) file within the CU file.

\section{0\% Random Sample of Optum Clinformatics Data Mart ${ }_{\circledast}$}

This database provides a nationally representative source of data on inpatient, outpatient and pharmacy claims, lab results, and some demographic characteristics. This dataset is a $10 \%$ random sample of health insurance claims from approximately 47 million individuals, $80 \%$ of whom were insured through their employment52. Data are available for periods between January 2007 and October 2017. We will use available data from October 2007 to October 2017. 


\section{CHAPTER 2}

\section{Economic Burden of Asthma- Chronic Obstructive Pulmonary Disease Overlap (ACO) among Older Adults in the United States}

\subsection{Abstract}

The objectives of this study are to estimate the excess economic burden of AsthmaChronic Obstructive Pulmonary Disease Overlap (ACO) among older adults in the United States and to evaluate the extent to which group differences in patient-level characteristics contribute to this excess economic burden. We used a cross-sectional study design with data from a nationally representative survey of Medicare beneficiaries (Medicare Current Beneficiary Survey) linked to Medicare fee-for-service claims. Economic burden was measured by total, third-party healthcare expenditures and patients' out-of-pocket spending (i.e. spending more than $10 \%$ of income on healthcare). Generalized Linear Models (GLM) and post-regression linear and non-linear decomposition methods were used to analyze the excess economic burden of ACO. Older adults with ACO had unfavorable profiles including a higher number of prescription medications utilized (mean $16.12 \pm 7.27$ vs. $7.53 \pm 5.32)$ and increased levels of fragmented care $(78.08 \pm 12.01$ vs. $66.80 \pm 22.17)$. Older adults with ACO had higher average total healthcare expenditures $(\$ 45,532$ vs. $\$ 12,743)$ and higher out-of-pocket spending burden (19\% vs. $8.5 \%)$ compared to those without asthma or COPD. Multivariable regression models indicated that the adjusted associations of ACO to economic burden remained positive and statistically significant. Nearly three-quarters of the excess total healthcare expenditures and $83 \%$ of the out-of-pocket spending burden were explained by differences in predisposing, enabling, need, personal healthcare practices, and external factors among the two groups. The higher number of unique medications and the increased incidence of fragmented care were the leading contributors of the excess 
economic burden among older adults with ACO. Interventions that reduce number of medications and fragmented care have the potential to reduce the excess economic burden among older adults with ACO.

\subsection{Introduction}

In the United States (US), chronic obstructive respiratory disease in older individuals occurs most often as COPD or asthma53. Among 15-45\% of older adults initially diagnosed with COPD or asthma, can later develop "Asthma-COPD overlap" (ACO) with symptoms consistent with each disease state 9. A significant disease burden and adverse health consequences exist for ACO patients compared to patients with either asthma only or COPD only. Prior research suggest that ACO patients experience higher acute respiratory events, outpatient visits, and hospitalizations compared to those with either asthma only or COPD only $12,13,53$. The prevalence rates of comorbid medical conditions and polypharmacy are also higher in ACO compared to either asthma or COPD 14 , suggesting that adults with ACO may constitute a significant economic burden.

However, current estimates of the economic burden of ACO in older adults are unknown. There have been only two studies in the US on the economic burden of ACO. The first study was conducted among enrollees of Medicare Advantage Plan in 2004. This study found that the average annual healthcare expenditures for the patients with ACO is higher than patients with COPD only 18 . The second study was a retrospective commercial claims analysis which reported that healthcare expenditures were twice as high for patients with ACO compared to those with asthma only between 2007-201017. Neither of these studies provided any information on average total healthcare expenditures, out-of-pocket (OOP) expenditures, and high OOP spending burden 
of older adults with ACO or compared economic burden with those who had no-asthma noCOPD (NANC).

Estimating the excess economic burden of ACO is important for several reasons. First, there is an emerging focus on achieving optimal healthcare with the triple aims of better health, higher quality, and lower costs54. For reducing overall healthcare costs, identification and management of high-cost conditions are very important55. Second, reducing costs also requires innovative practice models and payment reforms 56. For example, innovative payment models such as bundled payments for episodes of care need critical information on high-cost individuals as part of case mix adjustments 57. Third, estimating the excess economic burden can provide benchmarks for deriving cost-effectiveness of targeted interventions for management of ACO58. Finally, estimating the economic burden of ACO can translate disease burden into a universal metric (i.e. monetary value) which can be used for decision making by payers, policymakers, and other stakeholders 59.

In addition to excess economic burden, it is important to assess the high OOP spending burden because this can lead to poorer health-related behavior (e.g. poor medication adherence)60, poorer health outcomes, and increased future expenses61. High OOP spending burden is quite common among the older adults in the US. Although many older adults have health insurance coverage through Medicare, not all services are fully covered. This leaves many individuals paying for their healthcare through OOP payments. A study of older adults conducted by West Health and Gallup reported that the older adult population spent nearly $\$ 22$ billion from their savings to pay for healthcare, suggesting that older individuals may not have the financial resources to manage their health when they need it the most62. It has to be noted that nearly a quarter of Medicare beneficiaries have less than $\$ 15,000$ in total savings63. Although we do not 
have estimates of OOP payments among ACO patients, existing literature on OOP spending burden of either COPD or asthma suggest that OOP spending can be substantial. For example, the annual OOP costs of a single COPD inhaler per month was estimated to be about $\$ 900$ under a standard 2015 Part D plan64.

Although quantifying excess economic burden of ACO can provide needed information for decision making, it may not provide enough information to act on because costs are influenced by many factors including severity, care fragmentation65, age66, and presence of other chronic conditions.67 Similarly, high OOP spending burden is affected by many patient-level characteristics (e.g. age68, comorbidities69, income level70). Understanding the extent to which differences in patient-level factors drive the excess economic burden and high OOP spending burden of ACO is vital to case-mix adjustment, allocation of resources, payment reforms, costsharing, and cost reduction efforts.

Therefore, the main objectives of this study are to quantify the economic burden of ACO using data from a nationally representative sample of Medicare beneficiaries, and estimate the extent to which differences in characteristics among patients with ACO and NANC explained this economic burden.

\subsection{Methods}

\section{Study Design}

This study used a cross-sectional study design by using data from multiple years of linked fee-for-service Medicare claims with a nationally representative survey of Medicare beneficiaries.

\section{Data Source}


This study used the Medicare Current Beneficiary Survey (MCBS) linked to fee-for-service Medicare claims from 2006 through 2013. The MCBS is a rotating four-year panel survey of a nationally representative sample of individuals enrolled in Medicare Part A and B. The survey data sources provide information on direct medical costs, sources of payment, OOP payments, , health outcomes, clinical diseases, and sociodemographic of beneficiaries51. To improve the completeness and accuracy of medical costs and utilization, survey data are matched with feefor-service Medicare claims for making best of both claims and survey data worlds 49 . For this study, we used Medicare claims files for capturing information on health conditions and thirdparty payments. We also used "Cost and Use" (CU) survey files for deriving OOP burden and other variables (e.g. sex, age, race, prescription coverage, education level, disability status, smoking habit, and residency region).

\section{Study Sample}

The final analytical sample consisted of 38,089 person years and included communitydwelling Medicare beneficiaries, 65 years or older, alive throughout the calendar year, and enrolled in both Parts A and B fee-for-service during the calendar year. (Appendix 7.1)

\section{Measures}

Dependent Variables: Economic Burden

\section{Total HealthCare Expenditures}

All expenditures information in MCBS linked to Medicare claims files was extracted through a reconciliation process by combing survey information and Medicare claims files51. Linking these files provide a unique opportunity of cost analyses on the Medicare population compared to using either survey data or claims data alone49. Respondents to survey utilized calendars to record their medical events and were asked to save all their receipts from insurance 
companies, their receipts, and prescription bags provided by the pharmacy. Total healthcare expenditures were calculated by adding all the expenditures for inpatient visits, outpatient visits, prescription drugs, medical provider services, dental services, and other medical services. Sources of payment included both third-party expenditures and out-of-pocket (OOP) expenditures.

\section{Third-party Expenditures}

The third-party payers in this study included Medicare, Medicaid, private insurances, Veterans Affairs Health insurance, and other payers. We calculated third-party expenditures by subtracting OOP payments from total healthcare expenditures. To account for inflation of medical services, all the expenditures were converted to 2013-equivalent US dollars, by using the annual consumer price index of medical services 71.

High OOP Spending Burden of Individuals and Families

OOP expenditures in the MCBS represented amount spent by the patients or their families on healthcare, but not include insurance premium. To capture OOP expenditures, respondents of the MCBS were asked to record all the details of what they spent on healthcare bills and what they expected to spend on additional bills51. Respondents also reported their household income. OOP spending burden" was calculated as the ratio of OOP to income. Older adults who spent more than $10 \%$ of their income on healthcare, were classified as having high OOP burden.

Spending more than $10 \%$ of income on healthcare may compromise essential living expenses such as housing and food. As there is no consensus on the definition of OOP burden, we chose the $10 \%$ threshold based on previously published literature which reported that this threshold is above generally accepted affordability standards61,72. 
Key Independent Variable: Asthma/ COPD categories

The key independent variable of this study was asthma/COPD categories. Individuals with either asthma or COPD were identified by using the International Classification of Diseases, 9th edition diagnostic codes available in fee-for-service Medicare claims (Appendix 7.2). We used inpatient, outpatient, physician, skilled nursing home, and home healthcare claims. The diagnosis codes for identification of asthma and COPD cases have been used in previously welldesigned published research73,74. Individuals with a minimum of two outpatient visits (14 days apart) or one inpatient visit for asthma were considered to have asthma. Older adults with one inpatient or two outpatient visits (14 days apart) for COPD were categorized as individuals with COPD. Based on the presence of asthma or COPD, we created the following four asthma/COPD categories: 1) Asthma COPD Overlap (ACO); 2) Asthma only; 3) COPD only; and 4) No Asthma no COPD (NANC).

Other independent variables

The selection of other independent variables that could affect expenditures were derived from published literature 75,76 and guided by Andersen's Healthcare Behavioral Framework 50. Based on the model, the healthcare outcomes (in our study total healthcare expenditures) is affected by predisposing (age, sex, and race), enabling (marital status, education level, poverty status, prescription coverage, and fragmented care), need (number of chronic conditions, number of activities of daily living (ADL), and number of unique medications) factors, personal healthcare practices (obesity and smoking status) and external environment (e.g. residency region). For this study, the number of chronic conditions included cancer, diabetes, hypertension, heart disease, arthritis, and osteoporosis. We calculated fragmented care similar to previous studies77,78. 


\section{Statistical Analyses}

Patient characteristics by asthma/COPD categories were compared using chi-square test for categorical variables and ANOVA for continuous variables. The unadjusted and adjusted associations of asthma/COPD categories to economic burden were evaluated using generalized linear models (GLM). GLM with a gamma distribution and log-link function was used for total and third-party expenditures and GLM with binomial distribution and log-link function (i.e. logistic regression) was used to model high OOP burden. The robust estimates of excess burden of ACO were derived using the counterfactual recycled prediction. Separate models were used for total healthcare expenditures, third-party expenditures, and OOP expenditures. In all the models, the reference group was NANC. In the adjusted models, we controlled for predisposing, enabling, need factors, personal healthcare practices, and external environmental factors.

To estimate the extent to which differences in characteristics of individuals among the four groups (ACO, asthma only, COPD only, and NANC) contribute to the excess economic burden we used linear and non-linear decomposition analyses. In these analyses, we distinguish between explained and unexplained parts of the average difference in an outcome (example: total healthcare expenditures). The explained part was derived by taking the differences in average characteristics between two groups and weighting by regression co-efficient estimates from the pooled regression of fully adjusted model. Unexplained portion was derived from differences in regression-coefficients of the two groups weighted by average characteristics of groups.

Linear decomposition analysis followed the Blinder-Oaxaca approach for survey data using pooled regression weights. The decomposition analysis used log-transformed total healthcare expenditures as the dependent variable and assesses how much of the difference in log-transformed total healthcare expenditures between groups can be due to difference in 
characteristics (e.g. the predisposing, enabling, need, personal healthcare practices, and external environmental factors described above). For high OOP burden, we used the non-linear decomposition technique proposed by Fairlie79. We summarize results from the decomposition analysis by comparing NANC to: 1) ACO; 2) COPD only and 3) asthma only. All data management and analyses were conducted with SAS 9.4 (SAS Institute Inc., Cary, NC) and we used Stata 14 (StataCorp LLC, College Station, TX) for decomposition analyses.

\subsection{Results}

\section{Sample Description}

The study sample consisted of 38,089 person-years and included community-dwelling, full-year enrolled in fee-for-service Parts A and B, elderly (age > 65 years) Medicare beneficiaries. (Appendix 7.3) The prevalence of ACO, asthma only, COPD only conditions were $1.5 \%, 2.4 \%$, and $8.8 \%$ respectively between 2006 and 2013. ACO, asthma only, COPD only and NANC groups were significantly different in terms of all predisposing, enabling, need, personal healthcare practices, and external factors (Table 2.1). For example, individuals with ACO had the highest rates of poverty $(59.8 \%$ vs $44.2 \%)$ and higher levels of fragmented care $(78.08 \pm$ 12.01 vs $66.08 \pm 22.17)$ and higher number of unique medications $(16.12 \pm 7.27$ vs $7.53 \pm 5.32)$ as compared to NANC.

\section{Healthcare Expenditures}

The unadjusted and adjusted average annual total healthcare and third-party expenditures are provided in Figure 1. Average total, third-party and OOP expenditures were significantly higher for all Asthma/COPD categories compared to NANC. Medicare beneficiaries with ACO in comparison with NANC had almost 3.5-time higher unadjusted annual average total healthcare expenditures ( $\$ 45,532$ vs. $\$ 12,743)$, with an excess per patient cost of $\$ 32,789$ ( $\mathrm{P}$ 
$<0.001)$. The excess total healthcare expenditures COPD only and asthma only were $\$ 17,409$ and $\$ 10,760$ respectively. The excess third-party expenditures for ACO, COPD only and asthma only were: $\$ 31,113, \$ 16,383$, and $\$ 9,459$ respectively. Similar differences were observed in OOP payments (Figure 1).

Using recycled prediction, we observed that annual total healthcare expenditures remained significantly higher (Figure 2.1-b) for those with ACO $(\$ 22,968)$, yielding an excess cost of \$7,211 ( $\mathrm{P}<0.001)$. Adjusted annual third-party payers' expenditures were significantly higher $(\$ 6,871)$ for individuals with ACO as compared to NANC. Compared to older adults with asthma only, individuals with ACO had an excess total healthcare expenditure of $\$ 11,505$ ( $\mathrm{P}<0.001)$ and third-party payers' expenditures of $\$ 11,351(\mathrm{P}<0.001)$. Moreover, individuals with ACO had \$6,504 ( $\mathrm{P}<0.001)$ excess total healthcare expenditures and $\$ 6,273(\mathrm{P}<0.001)$ higher third-party payers' expenditures in comparison with those with COPD only.

Results from the linear decomposition analysis are summarized in Table 2. The average predicted log-transformed total healthcare expenditures for the ACO group was 10.393 units; the corresponding figure for NANC was 8.994 units, yielding a difference of 1.399 log-transformed expenditures. We found that differences in predisposing, enabling, need, personal healthcare practices, and external factors could explain $77 \%$ of this difference in average log-transformed total healthcare expenditures. Thus, if older adults with ACO were to have similar characteristics as those with NANC, the difference in log-transformed dollars would reduce to 8.99. Of the 1.399 unit difference, 1.081 units $(76.98 \%)$ were explained by the differences in characteristics. The number of unique medications (0.833 units, $77.30 \%)$ and fragmented care (16.08 units, $14.93 \%$ ) were explained by differences in other characteristics. 
Of the total difference in log-transformed costs (1.003) among COPD only and NANC group, 0.594 units (or 59.21\%) was explained by differences in the patient-level characteristics between the two groups. Similarly, of the total difference in log-transformed expenditures (0.735) among asthma only and NANC group, 0.655 units $(89.21 \%)$ was explained by differences in characteristics between asthma only and NANC groups. In all these comparisons, number of unique medications, need factors (i.e. number of chronic conditions and number of ADL) and fragmented care were the leading drivers of the excess economic burden.

\section{High OOP spending burden}

Figure 3.2 summarizes the prevalence of high OOP spending burden among the four asthma/COPD groups; $42 \%$ with ACO, 34\% with COPD and 29\% with asthma reported high OOP burden as compared to $17 \%$ among individuals with NANC. In the unadjusted logistic regression models, the odds of high OOP spending burden was significantly higher among individuals with $\mathrm{ACO}(\mathrm{UOR}=3.51,95 \% \mathrm{CI}=[2.96,4.16], \mathrm{P}<0.001)$, asthma only $(\mathrm{UOR}=1.99$, $95 \% \mathrm{CI}=[1.68,2.36], \mathrm{P}<0.001)$, and COPD only $(\mathrm{UOR}=2.40,95 \% \mathrm{CI}=[2.20,2.61], \mathrm{P}<0.001)$ as compared to the NANC group. In the adjusted logistic regression models, the association remained significant. The odds of high OOP spending burden was significantly higher among individuals with $\mathrm{ACO}(\mathrm{AOR}=3.51,95 \% \mathrm{CI}=[2.96,4.16], \mathrm{P}<0.001)$ and $\mathrm{COPD}$ only $(\mathrm{AOR}=$ $3.51,95 \% \mathrm{CI}=[2.96,4.16], \mathrm{P}<0.001)$ as compared to the NANC group.

Based on non-linear decomposition analysis, we found that of the 23.53 percentage point difference in high OOP burden, 19.59 percentage points were explained by the differences in the characteristics between the ACO and the NANC group (Table 2.2). Thus, if older adults with ACO were to have similar characteristics as those of the NANC group, the difference in OOP burden would be reduced to 18.5 percentage points. Of this total difference, 13.18 percentage 
points (or 67.29\%) was explained by the number of unique medications. Similar findings were observed when asthma only group was compared to NANC and the COPD only group was compared to NANC.

\subsection{Discussion}

This study examined the excess economic burden associated with ACO among older adults in the US, using multiple years of a nationally representative sample of Medicare beneficiaries. In this study, the overall prevalence of ACO was $1.5 \%$. Although no study has reported the specific prevalence of ACO among Medicare beneficiaries, this estimate is less than the $3.8 \%$ which was previously reported by Kumbhare et al for (65-85 years old) older adults in the United States14. Possible explanations of this difference are methods of identification and time period. While this study used fee-for-service Medicare claims for identification of ACO during a calendar year, Kumbhare et. Al. used "ever diagnosed" using self-reports. Thus, we may have captured only older adults who had currently active asthma and COPD and had diagnoses updated in their medical claims.

In terms of projected total healthcare expenditures, nearly two billion dollars (2013USD) was spent on the healthcare of older community-dwelling adults with ACO (weighted number of ACO adults in $2013=42,984$ multiplied by the average per-capita cost $\$ 45,532)$. In terms of the excess economic burden, individuals with ACO had almost four, two, and 1.5 times higher expenditures compared to individuals with NANC, asthma only, and COPD only, respectively. Even after controlling for multiple determinants of expenditures, the presence of ACO was associated with significantly higher expenditures in comparison with individuals with NANC, asthma only, or COPD only, consistent with previously published studies that compared per- 
capita expenditures of individuals with ACO to those with asthma only 17 or those with COPD only18.

The excess expenditures associated with ACO is not surprising because studies have documented increasing expenditures with increasing number of chronic conditions 80.

Furthermore, our finding that ACO patients had higher economic burden compared to those with asthma only or COPD only may be explained through higher rates of uncontrolled disease states, respiratory infections, and exacerbations, which may lead to higher expenditures. While healthcare utilization and reasoning for expenditure was not directly reviewed in this study, a prior study of 17,088 COPD patients in Taiwan reported that ACO patients experienced almost twice as many acute respiratory events (e.g. acute respiratory failure, pneumonia, and acute exacerbation) than did the rest of the COPD cohort12. This higher event rates undoubtedly lead to higher healthcare resource utilization and overall higher direct costs.

In this study, those with ACO had higher odds of high OOP spending burden compared to all other groups in both unadjusted and adjusted models. This high burden could be due to cost-sharing of Medicare-covered services, including prescription drugs and payments for noncovered services by Medicare. OOP burden may be particularly high among individuals with ACO because of historically high cost-sharing of inhaler medications. Based on data from nationwide Part D plans, it has been estimated that mean OOP expenditures were $\$ 30-\$ 105$ per inhaler in 201564. According to this study, a typical older Medicare beneficiary who use 2-3 inhalers per month have annual OOP expenditures of at least $\$ 1,622-\$ 2,811$ for their inhalers.

Individuals with ACO may have greater need for some of the services not covered by Medicare compared to NANC as they are higher risk of these conditions. For example, individuals with ACO have significantly higher odds $(\mathrm{AOR}=1.82,95 \% \mathrm{CI}=[1.59,2.08]$, 
$\mathrm{P}<0.001$ ) of removing all teeth compared to $\mathrm{NANC} 81$, suggesting greater spending on oral healthcare by patients. It has also been reported that COPD patients are vulnerable to low-value cares2, which may increase OOP spending of families and patients through cost-sharing. Medicare reform proposals that focus on value-based healthcare that balance the value of health services to the OOP spending burden of patients (vs. quantity of care) may reduce the excess economic burden of patients.

The current study used linear and non-linear decomposition analyses to examine the excess economic burden of chronic respiratory conditions, one of the top four conditions of public health concern designated by the World Health Organization (WHO) 83 . The other three conditions are cardiovascular disease, diabetes, and cancer. It is important to note that older adults with chronic respiratory conditions also have higher rates of CVD, diabetes, and cancer. A population survey among ( $\geq 18$ years) adults in the US also found that the adjusted odds of six chronic conditions (e.g. cardiovascular diseases, diabetes, and depression) is higher in ACO compared to asthma only and COPD only 14. In our study, 91\% of ACO patients had more than two other chronic conditions which was higher than asthma only (89\%), COPD only (87\%) and NANC (76\%) groups. Our decomposition analysis suggested that patient-level characteristics such as higher number of co-existing chronic conditions and related characteristics (i.e., higher number of unique medications and higher levels of fragmented care) were the leading drivers of the excess economic burden among ACO patients. For example, receiving care from several different providers often results in gaps in communication between providers which can lead to suboptimal care and polypharmacy75,84. Prior studies suggest that more fragmented care is associated with more diagnostic tests, more low-value care, and lower patient satisfaction85-87. Previous studies further suggest the associations of fragmented care and higher number of emergency department (ED) visits, higher hospitalization rates, and higher expenditures $77,88$.

Taken together, our study findings highlight the need for a "holistic management" of older adults with chronic respiratory conditions, specifically ACO, asthma, and COPD. Given that those with chronic 
respiratory conditions may need to see multiple providers and have a high medication burden, the risks of fragmented care and drug-disease and drug-drug interactions are very high. These factors often lead to greater economic burden, as evidenced by our study. Although the CMS began reimbursing providers for providing coordinated care for those with multiple conditions in 201589 , it remains to be seen whether such fiscal incentives for care coordination have resulted in less fragmented care and reduced healthcare expenditures. In this context, patients with chronic respiratory conditions (ACO, asthma, and COPD) may need to be managed in a patient-centered medical home model which focuses care that is "comprehensive, team-based, coordinated, accessible, and focused on quality and safety." 90. As documented in published literature, pharmacists can play an important role in medical homes by reducing unnecessary medications, counseling patients and/or caregivers, reviewing medication regimens and inhaler techniques, and completing adherence assessments91-93. Such management may improve not only outcomes but also lower the economic burden of patients, payers, and health systems.

Summarily, our findings have policy and program implications. Our study findings highlight the need to develop guidelines for the management of overlapping chronic conditions. Applying "singledisease focused" clinical practice guidelines can result in missing or inappropriate therapies and adverse drug-drug and drug-disease interactions. A systematic review of recommendations in 12 UK national clinical guidelines notes that potentially serious drug-drug interactions were common94. Adherence to guidelines intended for single-diseases in those with multiple chronic conditions can also generate more low-value care and lead to higher healthcare expenditures the patient, payors, and the society as holistic, big-picture care may be lacking 95,96.

\subsection{Limitation}

As with most studies, this study also had some limitations. The first limitation was the lack of availability of data from recent years. Notwithstanding this limitation, we used the most recently available data of a nationally representative large sample of elderly Medicare beneficiaries. Another limitation was the lack of information on the onset and severity of 
diseases. To overcome this, we used ADL status as a proxy of overall severity of diseases. Furthermore, the findings of the current study cannot be generalizable to all older adults in the US, although it is generalizable to the majority of older adults (community-dwelling Medicare beneficiaries enrolled in fee-for-service Part A and B).

\subsection{Conclusion}

This study is the first to document the excess economic burden of ACO among elderly Medicare beneficiaries. ACO among elderly Medicare beneficiaries was associated with substantial total healthcare expenditures, third-party payers' expenditure and high OOP spending burden, even after adjusting with a comprehensive list of variables. Post regression decomposition analyses revealed that the challenges associated with multiple chronic conditions, specifically use of multiple medications and high levels of fragmented care were the leading drivers of the excess economic burden. Individual, policy, and program interventions that facilitate coordination of care may reduce the excess economic burden of older adults with chronic respiratory conditions, specifically ACO, asthma, or COPD. 
Table 2.1.

Unweighted N and Weighted Percentages of Characteristics by Asthma /COPD Categories (Column \%) Older (age $\geq 65$ years) Medicare Beneficiaries

Medicare Current Beneficiary Survey, 2006-2013

\begin{tabular}{|c|c|c|c|c|c|c|c|c|c|}
\hline & \multicolumn{2}{|c|}{$\begin{array}{l}\text { Asthma-COPD } \\
\text { Overlap }\end{array}$} & \multicolumn{2}{|c|}{ Asthma only } & \multicolumn{2}{|c|}{ COPD only } & \multicolumn{2}{|c|}{$\begin{array}{l}\text { Neither Asthma } \\
\text { Nor COPD }\end{array}$} & \multirow[t]{2}{*}{ Sig } \\
\hline & $\mathbf{N}$ & Wt. \% & $\mathbf{N}$ & Wt. \% & $\mathbf{N}$ & Wt. \% & $\mathbf{N}$ & Wt. \% & \\
\hline ALL & 589 & 1.5 & 860 & 2.4 & 3,518 & 8.8 & $\mathbf{3 3 , 1 2 2}$ & 87.3 & \\
\hline Sex & & & & & & & & & **** \\
\hline Female & 389 & 67.0 & 658 & 77.6 & 1,719 & 49.7 & 18,703 & 56.1 & \\
\hline Male & 200 & 33.0 & 202 & 22.4 & 1,799 & 50.3 & 14,419 & 43.9 & \\
\hline Age in Years & & & & & & & & & *** \\
\hline $65-74$ & 205 & 39.5 & 397 & 53.7 & 1,215 & 40.9 & 13,290 & 48.1 & \\
\hline $75-84$ & 270 & 44.1 & 323 & 34 & 1,642 & 43.7 & 13,413 & 36.8 & \\
\hline 85 and above & 114 & 16.4 & 140 & 12.3 & 661 & 15.4 & 6,419 & 15.1 & \\
\hline Race/Ethnicity & & & & & & & & & $* * *$ \\
\hline White & 451 & 76.7 & 663 & 78.3 & 3,044 & 86.7 & 27,371 & 82.6 & \\
\hline Black & 47 & 8.0 & 85 & 9.5 & 168 & 4.7 & 2,303 & 6.8 & \\
\hline Latino & 48 & 7.6 & 60 & 6.5 & 146 & 4.0 & 1,891 & 5.9 & \\
\hline Other race & 42 & 7.7 & 52 & 5.7 & 153 & 4.6 & 1,486 & 4.8 & \\
\hline Marital Status & & & & & & & & & **** \\
\hline Married & 224 & 39.3 & 405 & 48 & 1,690 & 49.3 & 17,456 & 55.2 & \\
\hline Not married & 364 & 60.7 & 454 & 52 & 1,824 & 50.7 & 15,656 & 44.8 & \\
\hline \multicolumn{10}{|l|}{ Education } \\
\hline Less than high school & 194 & 31.6 & 178 & 19.3 & 1,150 & 31.7 & 7,734 & 21.3 & *** \\
\hline High school & 218 & 37.8 & 287 & 31.3 & 1,284 & 36.4 & 11,962 & 35.6 & \\
\hline Above high school & 175 & 30.6 & 393 & 49.4 & 1,072 & 31.9 & 13,295 & 43.1 & \\
\hline Poverty Status & & & & & & & & & *** \\
\hline Less than $200 \%$ FPL & 364 & 59.8 & 415 & 44.9 & 2,077 & 57.7 & 15,526 & 44.2 & \\
\hline At least $200 \%$ FPL & 225 & 40.2 & 445 & 55.1 & 1,441 & 42.3 & 17,596 & 55.8 & \\
\hline Prescription Coverage & & & & & & & & & *** \\
\hline Yes & 414 & 70.6 & 590 & 67.0 & 2,299 & 65.2 & 20,397 & 60.9 & \\
\hline No & 175 & 29.4 & 270 & 33.0 & 1,219 & 34.8 & 12,725 & 39.1 & \\
\hline \% Fragmented Care & $78.08 \dagger$ & $12.01 \%$ & $75.06 \dagger$ & $14.65 \%$ & $73.88 \dagger$ & $16.62 \%$ & $66.80 \dagger$ & $22.17+$ & $* * *$ \\
\hline \# Other Chronic Conditions & & & & & & & & & **** \\
\hline $0-1$ & 53 & 9.0 & 93 & 11.0 & 419 & 13.0 & 7,122 & 23.6 & \\
\hline $2-3$ & 273 & 47.6 & 471 & 56.4 & 1,754 & 50.7 & 17,539 & 53.4 & \\
\hline $4-6$ & 250 & 43.3 & 285 & 32.6 & 1,272 & 36.3 & 8,035 & 23.0 & \\
\hline \# ADL & & & & & & & & & $* * *$ \\
\hline 0 & 269 & 45.4 & 544 & 64.5 & 1,830 & 52.8 & 23,532 & 73.4 & \\
\hline $1-2$ & 192 & 34.0 & 213 & 25.0 & 1,096 & 31.0 & 6,683 & 19.0 & \\
\hline $3-6$ & 123 & 20.6 & 102 & 10.5 & 573 & 16.2 & 2,793 & 7.6 & \\
\hline $\begin{array}{l}\text { \# Unique Medications } \\
\text { Obesity }\end{array}$ & $16.12 \dagger$ & $7.27 t$ & $12.73 \dagger$ & $6.29+$ & $12.57 \dagger$ & $6.75 t$ & $7.53 \dagger$ & $5.32+$ & $\begin{array}{l}* * * \\
* * *\end{array}$ \\
\hline Yes & 207 & 38.5 & 298 & 36.5 & 897 & 26.8 & 7597 & 24.0 & \\
\hline No & 381 & 61.3 & 550 & 62.1 & 2588 & 72.2 & 25207 & 75.0 & \\
\hline Smoking Habit & & & & & & & & & *** \\
\hline Current/Past & 429 & 73.7 & 410 & 48.7 & 2943 & 84.4 & 18256 & 56.0 & \\
\hline Never & 154 & 25.3 & 449 & 51.2 & 556 & 15.0 & 14762 & 43.7 & \\
\hline Residency Region & & & & & & & & & *** \\
\hline Northeast & 118 & 23.3 & 170 & 22.6 & 578 & 17.9 & 5,115 & 17.3 & \\
\hline South & 126 & 20.4 & 189 & 21.8 & 873 & 23.8 & 8,321 & 23.9 & \\
\hline Midwest & 250 & 39.5 & 355 & 38.3 & 1,668 & 46.3 & 14,104 & 40.3 & \\
\hline West & 95 & 16.9 & 146 & 17.4 & 399 & 11.9 & 5,582 & 18.4 & \\
\hline
\end{tabular}

Note: Based on 38,089 person-years of community-dwelling elderly (age $\geq 65$ years) Medicare beneficiaries, continuously enrolled in fee-for-service Part A and Part B during the calendar year and alive during the calendar year.

Significant group differences in asthma-COPD status were based on Rao-Scott chi-square tests. ANOVA test was used for number of unique medications and \% fragmented care. 
ADL: Activities of Daily Living; FPL: federal poverty level; N: unweighted number; Sig: significance; Wt: weighted; \%: Percentage; $\dagger$ : Mean; $¥$ : Standard Error; ***p<.001, ; **.001 $\leq \mathrm{p}<.01 ; * .01 \leq \mathrm{p}<.05$ 
Figure 2.1.

Total Healthcare Expenditures and High Out-of-Pocket Burden by Asthma/ COPD Categories

Older (age $\geq 65$ years) Medicare Beneficiaries

Medicare Current Beneficiary Survey, 2006-2013

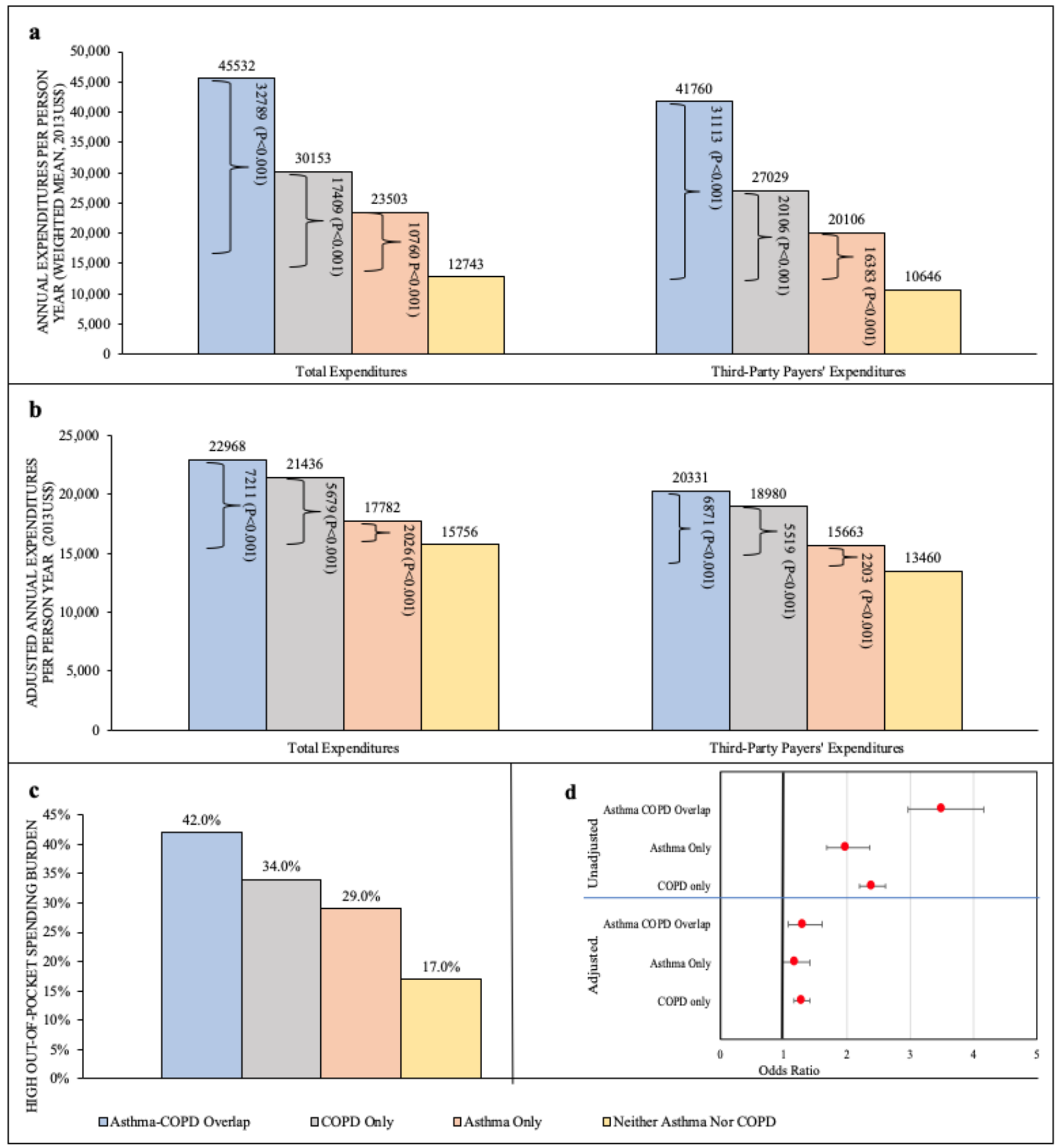

Note (a) Unadjusted expenditures, (b) Adjusted expenditures from recycle prediction" group, (c) high OOP spending burden percentage (d) Unadjusted and adjusted odds ratio of high OOP spending burden by asthma/COPD categories

Based on 38,089 elderly Medicare beneficiaries who were observed between years 2006 and 2013. The variables in the adjusted models were selected based on Andersen's healthcare behavioral model and include sex, age, race, marital status, poverty, education, prescription coverage, fragmented care, \# ADL, \# chronic conditions, \# unique medications, overweight, smoking habit, and residency region.

Abbreviations: ADL: Activity Daily Limitation; COPD: chronic obstructive pulmonary disease; OOP: Out-of-Pocket; Log: natural logarithm; \#: number; \$: Dollar; \%: Percentage; +: Excess cost comparing to no-asthma no-COPD; 
Table 2.2

Post-Regression Decomposition Analyses

Older (age $\geq 65$ years) Medicare Beneficiaries

Medicare Current Beneficiary Survey, 2006-2013

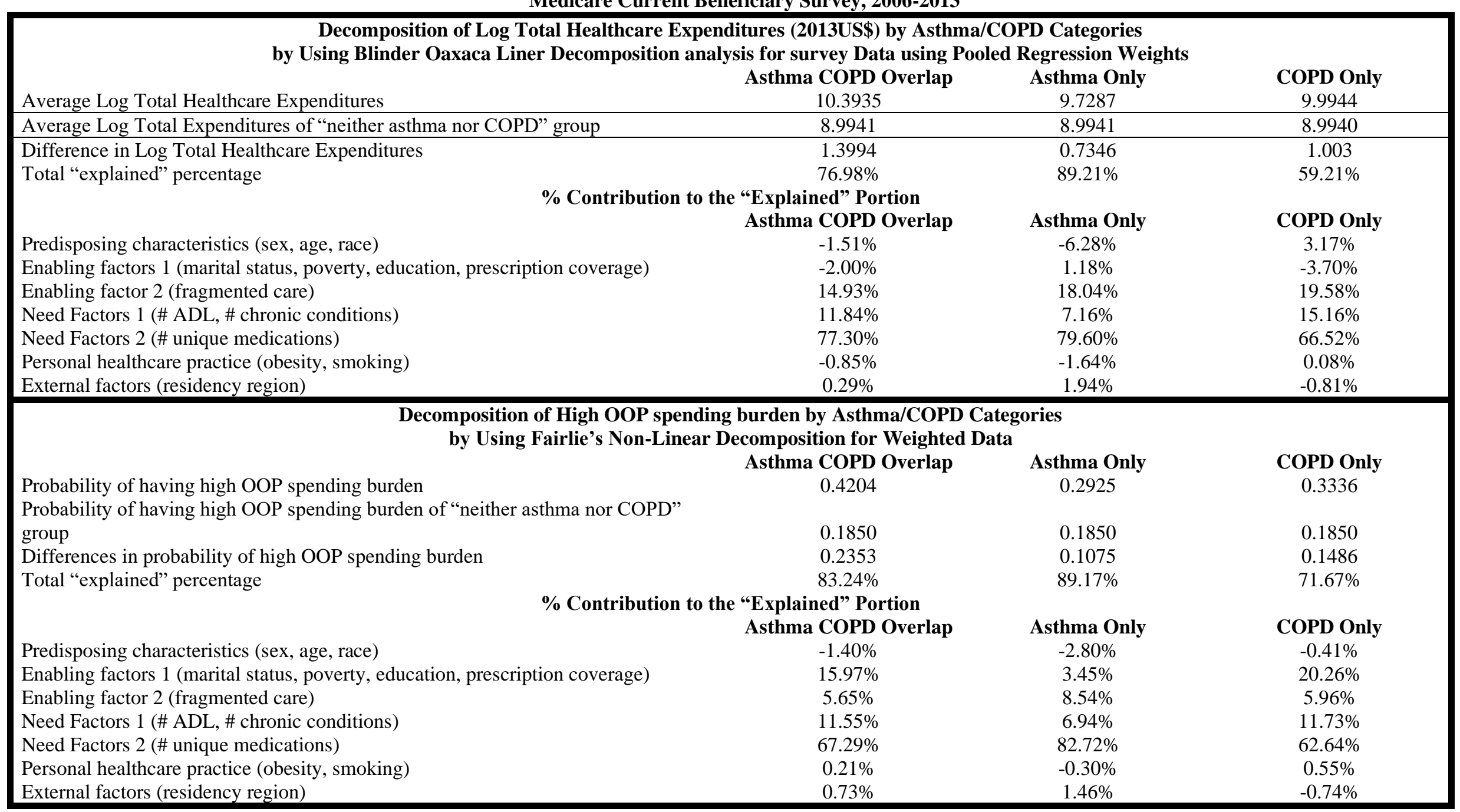

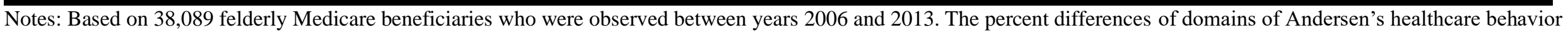
model are illustrated in the model.

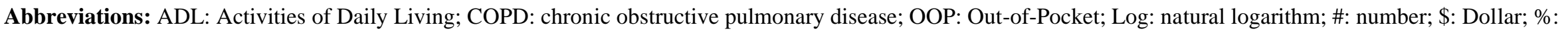
Percentage. 


\section{CHAPTER 3}

\section{Asthma-Chronic Obstructive Pulmonary Disease Overlap and Cost-Related Medication}

Nonadherence among Older Adults in the United States

\subsection{Abstract}

Objective: Cost-related medication non-adherence (CRN) can negatively impact health outcomes in older adults with asthma-chronic obstructive pulmonary disease (COPD) overlap (ACO) by reducing access and adherence to essential medications. The objective of this study is to examine the association of ACO to any $\mathrm{CRN}$ and specific forms of CRN among a nationally representative sample of older (age $\geq 65$ years) adults.

Methods: We adopted a cross-sectional study design using data from pooled cross-sectional Medicare Current Beneficiary Surveys (2006-2013) and linked fee-for-service Medicare claims. Unadjusted and adjusted logistic regressions that accounted for the complex survey design examined the association of ACO to any CRN and specific forms of CRN.

Results: Among older adults with ACO, $16 \%$ reported any CRN. The most common form of CRN was "failing to get prescription". As compared to older adults with no asthma and no $\mathrm{COPD}$, those with $\mathrm{ACO}$ were more likely to report any $\mathrm{CRN}(\mathrm{AOR}=1.50,95 \% \mathrm{CI}=[1.14$, 1.96]) and all forms of CRN. However, when the number of unique medications were added to the model, there were no statistically significant differences in CRN between the two groups. Conclusions: Older adults with ACO represent a vulnerable population with increased risk for CRN. Multiple factors can contribute to CRN including: a higher number of prescribed medications, multiple co-morbidities, and cost of therapies. Deprescribing - as appropriate - is an opportunity and process which could reduce the risk of CRN among the older Medicare beneficiaries with ACO. 


\subsection{Introduction}

Asthma and chronic obstructive pulmonary disease (COPD) are the two most common respiratory diseases worldwide. Combined, more than 435 million adults suffer from asthma and COPD 1,2 . In many cases where airway obstruction is noted, asthma and COPD can coexist as "Asthma-COPD overlap" (ACO), which can be as high as 50\% among elderly (age $\geq 80$ years) patients with either asthma or COPD 10. Treatment for both asthma and COPD focuses on symptom management rather than clinical cure; similarly, ACO is managed with pharmacotherapy recommended in both disease states. Adherence is critical in ACO as therapy is primarily maintenance therapy (i.e. taken daily) versus as needed to maximize effects on reducing inflammation, bronchoconstriction, and sputum production. 20,97. However, studies demonstrate many individuals with either asthma or COPD do not adhere to prescribed medication regimens with non-adherence rates often $\geq 20 \% 26,29,30$. While many factors influence medication adherence, older adults with chronic conditions often report cost-related barriers as a reason for medication nonadherence 98-102. High out-of-pocket (OOP) prescription costs can overwhelm some older adults compelling them to adopt cost-coping strategies 103 such as (1) failing to fill a prescription; (2) delaying filling prescriptions; (3) skipping medication doses; and (4) using less than prescribed doses of a medication 104.

Although there are no published studies on cost-related non-adherence (CRN) among older adults with ACO, it is expected that ACO individuals may be particularly vulnerable to CRN based on studies focusing on either asthma or COPD. In a 2004 study of disease-specific rates of CRN by Piette et al105, asthma and COPD were among diseases with the highest rates of CRN. Castaldi et al estimated that over one third of older adults with chronic pulmonary diseases reported $\mathrm{CRN}_{32}$. However, this study did not compare the risk of CRN for different types of chronic pulmonary diseases including ACO.

Insight into the association of CRN to ACO is important because CRN has downstream effects and can lead to higher healthcare utilization76, morbidity98, and mortality106. Information on contributors to CRN among adults with ACO can be used to develop a portfolio of programs targeting these contributors 
and subsequently help improve adherence and reduce the risk of mortality and morbidity among older adults with ACO.

Therefore, our primary objective was to assess the association of ACO to CRN as compared to no-asthma and no-COPD and explore the factors that may affect the association of ACO to CRN among older adults (age $\geq 65$ years), using a nationally representative sample of Medicare beneficiaries in the US.

\subsection{Methods}

\section{Study Design}

We adopted a cross-sectional study design with data from the annual Medicare Current Beneficiary Survey (MCBS) from 2006 through 2013.

\section{Data Source}

The data sources for this study were the Medicare Current Beneficiary Survey (MCBS) and fee-for-service Medicare claims that are linked to the survey respondents. The MCBS is a rotating panel survey of a nationally representative sample of the Medicare population and has been carried out since 1991. The goals of the survey are to collect information on healthcare access, expenditures, sources of payment, health outcomes, health status, and satisfaction with care of Medicare beneficiaries51. To improve the accuracy and completeness of healthcare use and costs, survey data are matched, reconciled, and supplemented with fee-for-service Medicare claims creating the best of both survey and claims data worlds49.

From 1992 to 2013, the MCBS released two data files, the "Access to Care" (AC) and the "Cost and Use" (CU) files, annually. While both AC and CU files include some common elements, AC files provide additional information on access to care, information needs, patientprovider relationships, and satisfaction with care on "always enrolled" Medicare beneficiaries. CU files include detailed data on reconciled cost and use information on "ever enrolled" 
Medicare beneficiaries 51. Medicare claims have information on all reimbursed healthcare encounters of fee-for-service Medicare beneficiaries including International Classification of Diseases 9th Edition (ICD-9) codes, payments, procedures, and other treatments.

For this study, we used AC and CU files and fee-for-service Medicare claims from 2006 to 2013. We selected these years because of the introduction of Medicare Part D program beginning in 2006. We used AC files to capture information on CRN. We used CU files for deriving other variables. We identified asthma-COPD status by using fee-for-service inpatient, outpatient, physician, and home healthcare Medicare claims files.

\section{Study Sample}

The study sample included community-dwelling elderly (age $\geq 65$ years) Medicare beneficiaries who were enrolled in fee-for-service (Parts A and B) during the calendar year and alive throughout the calendar year. We further restricted our study sample to those with available information in both $\mathrm{AC}$ and $\mathrm{CU}$ files of the MCBS and those without missing data for CRN measures $(\mathrm{N}=37,571)$. (Appendix 7.7)

\section{Measures}

\section{Dependent Variable: Any CRN (Yes/No) and Specific Forms of CRN}

In MCBS, four questions queried CRN-related behaviors. The validity and reliability of using these questions for measuring $\mathrm{CRN}$ have been confirmed in previous studies 107-109. Respondents were asked, "Please tell me how often during (CURRENT YEAR) you did any of the following behaviors (1) decided not to fill a prescription because it cost too much, (2) delayed getting a prescription filled because the medicine cost too much, (3) skipped doses to make the medicine last longer, (4) taken smaller doses of a medicine to make the medicine last longer". The answers were recorded on a 3-point scale (often, sometimes, and never). If 
respondents answered "often or sometimes" to any of these questions, the respondents were considered to have any CRN, and those who responded never to all the questions were considered to have no CRN. For all specific forms of CRN, we combined "often" and "sometimes" to create indicator variables (Yes/No).

\section{Key Independent Variable: Asthma and COPD categories}

The key independent variable was asthma-COPD status. The first step of preparing this variable was identification of individuals with either asthma or COPD by using the ICD-9 codes available in Medicare claims (Appendix 7.2). These diagnosis codes for asthma and COPD have been utilized in previously published research73,74. Individuals with at least one inpatient visit or two outpatient visits (at least 14 days apart) for either asthma or COPD codes were considered to have diagnosed asthma or COPD. Based on the presence of asthma or COPD, we created the following four categories: 1) Asthma and COPD (ACO); 2) Asthma without COPD; 3 ) No asthma with COPD; and 4) No asthma and No COPD.

\section{Other independent Variables}

We used Anderson's health behavior model as well as published literature to guide the selection of other independent variables associated with CRN. We selected Andersen behavior model for this study as it gives us a comprehensive list of factors associated with CRN. Per the framework, CRN may be affected by: 1) pre-disposing variables (Sex (Male/female); age (6574/75-84/ 85 years or older); race/ethnicity (White/Black/Hispanic/or other races)); 2) enabling factors (marital status (married/not married); education level (less than high school/high school/and above high school); poverty status [based on income relative to the level of federal poverty line $(\mathrm{FPL})](<200 \%$ of FPL $>=200 \%$ of FPL $)$ ); high burden of OOP prescription cost ([annual OOP prescription costs > annual 10\% of income] (yes/no); high burden of OOP medical costs [annual OOP medical costs > annual 10\% of income] (yes/no); and prescription drug 
coverage (yes/no)); 3) need factors (the number (1-6) of chronic conditions (cancer, diabetes, hypertension, heart disease, arthritis, and osteoporosis)); the number of unique medications; personal health practices (obesity status (yes/no); and smoking status (neversmoker/former/current smoker).

\section{Statistical analyses}

Unadjusted group differences in terms of asthma-COPD status were tested with RaoScott Chi-square tests. We used separate logistic regression models to examine the unadjusted and adjusted associations between asthma-COPD status and CRN (i.e. any CRN and specific forms of CRN). Results are presented in terms of unadjusted (UOR) and adjusted odds ratios (AOR) and 95\% confidence intervals (CI). All analyses utilized the SURVEY procedures of Statistical Analysis Software (SAS $®$ ) version 9.4 to account for the complex survey design of MCBS.

\subsection{Results}

The study sample consisted of 37,571 person-years and included elderly (age $\geq 65$ years) community-dwelling, full-year fee-for-service Parts A and B Medicare beneficiaries, without missing data on CRN measures between 2006 and 2013. (Appendix 7.8) The overall prevalence of ACO, asthma-only, and COPD-only was $1.5 \%, 2.3 \%$, and $8.8 \%$ respectively during the study period.

There were significant differences between asthma-COPD status groups in terms of sex, age, race/ethnicity, marital status, education level, poverty status, prescription coverage, high burden of prescription cost, high burden of medical cost, the number of chronic conditions, and number of unique medications (Table 3.1). For example, individuals with ACO have the highest rates of high burden of OOP prescription costs $(9.3 \%)$ and OOP medical costs (28.3\%) compared 
to individuals with asthma-only (6.2\% \& 17.1\%), COPD-only (9.0\% \& 20.9\%), and no-asthma no-COPD (4.0\% and $11.0 \%)$. The number of unique medications was the highest among individuals with ACO (16.21 \pm 0.34$)$ compared to those with asthma-only (12.74 \pm 0.29$)$, COPDonly $(12.51 \pm 0.17)$, and no-asthma no-COPD $(7.37 \pm 0.06)$.

Unadjusted and Adjusted Associations of ACO to any CRN

The prevalence of any CRN was 9.7\% among older Medicare beneficiaries. Individuals with ACO had the highest percentage of any CRN (16.0\%) compared with individuals with asthma-only (14.5\%), COPD-only (13.0\%), and no-asthma no-COPD (9.1\%) (Figure 3.1). Compared to individuals with no-asthma no-COPD, individuals with ACO (UOR $=1.89,95 \%$ $\mathrm{CI}=[1.45,2.45])$ were more likely to report CRN (Table 3.2). In models without adjustments for number of unique medications, individuals with $\mathrm{ACO}$ were more likely to report $(\mathrm{AOR}=$ $1.50,95 \% \mathrm{CI}=[1.14,1.96])$ any $\mathrm{CRN}$ compared to individuals with no-asthma no-COPD (Table 2). In the fully adjusted model for any CRN, those with ACO were as likely as those with no asthma and COPD to report $\mathrm{CRN}(\mathrm{AOR}=1.23,95 \% \mathrm{CI}=[0.92,1.63], \mathrm{p}=0.15)$. Association of ACO to specific forms of CRN

Those with ACO had the highest percentages of three specific forms of CRN including: failing to fill prescription $(10.1 \%)$, delaying filling prescription $(7.9 \%)$, and skipping medication doses (6.9\%) (Figure 3.1). In the unadjusted and adjusted models, without number of unique medications, individuals with ACO had higher odds of "failing to fill prescription", "delaying filling prescriptions", and "skipping medication doses", compared with individuals with noasthma no-COPD (Table 3.3).

In the fully adjusted model that included the number of medications as one of the independent variables, those with $\mathrm{ACO}(\mathrm{AOR}=1.43,95 \% \mathrm{CI}=[0.99,2.07], \mathrm{p}=0.05)$, had 
marginally higher odds of "failing to fill prescription" compared to those with no asthma and no COPD. In the fully adjusted models for other specific forms of CRN, we did not observe statistically significant differences among adults with ACO and adults with no asthma and no COPD.

Comparison ACO with asthma-only and COPD-only groups

Comparisons were also made among asthma or COPD categories (asthma only, COPD only). As indicated in Table 3.2, we also used COPD-only or asthma-only as reference groups. In all the models, there were no statistically significant group differences among asthma only, COPD only, and ACO.

\subsection{Discussion}

One in six older Medicare beneficiaries with ACO reported any CRN between 2006 and 2013. The most prevalent form of CRN was "failing to fill prescription," which was almost twice as high among Medicare beneficiaries with ACO compare to those with no-asthma and noCOPD.

Older adults with ACO were more likely to report any CRN compared to older adults with no-asthma no-COPD; while not directly studied, this likely has significant later-in-life morbidity and mortality consequences76,98. This is not surprising because older adults who had high OOP burden compared to those with neither asthma nor COPD and high OOP burden was significantly associated with CRN. The high OOP burden among adults with ACO can be explained by the high cost of brand-name inhaler therapies and the high medication burden per individualized regimen to adequately manage ACO (often 2 to 3 inhaler therapies). Although, we did not distinguish between asthma and COPD-related medications, we speculate that those with ACO need to use inhalers for the management of ACO, as these are first line therapy per the 
most recent guidelines 110 . The cost of inhalers dramatically increased in 2008, after the banning of chlorofluorocarbon propellants111. This led to phasing out generic inhalers, leaving only maintenance, brand-name inhalers112. A study by Tseng et al indicated that although inhalers were universally covered by Medicare Part D plans, it required high cost-sharing by Medicare beneficiaries64. This can be seen as patients experience higher annual plan deductibles and elevated copay costs (inhalers are often included in higher tiers of formularies because of their brand status) despite Medicare covering a portion of the prescription. For instance, using a single inhaler each month had projected annual OOP costs of $\$ 900$ under a standard Part D plan in 201564. Our study findings suggest that policy makers may need to re-evaluate coverage of inhalers under Part D plans.

In our study, increased risk of any $\mathrm{CRN}$ among $\mathrm{ACO}$ individuals compared to those with no-asthma or no-COPD is apparent by adjusting all factors without number of unique medications. However, the inclusion of number of unique medications attenuated this association, suggesting that number of medications may be a key link between ACO and CRN. Notably, the mean number of unique medications is approximately 16 among individuals with ACO which is significantly higher compared to individuals with asthma-only ( 13), COPD-only $(\sim 12.5)$, and no-asthma no-COPD ( 7). Individuals with ACO have a higher number of unique medications due to two following reasons: (1) individuals with ACO may need to use a higher number of respiratory medications to adequately control respiratory symptoms. The Latin American Project for the Investigation of Obstructive Lung Disease (PLATINO) study found that $49 \%$ of individuals with ACO used respiratory medications significantly higher than individuals with asthma-only (38\%) or COPD-only (20\%)13; (2) ACO individuals may need to use a greater number of non-respiratory medications due to higher risk of comorbid medical 
conditions. In our study, $43 \%$ of ACO individuals had 4-6 other chronic conditions, while this number was lower among individuals with asthma-only (33\%), COPD-only (36\%), and noasthma no-COPD (23\%). A population survey in the US similarly found that $90 \%$ of individuals with ACO had at least one other co-existing condition (compared with $71 \%$ in asthma-only and $84 \%$ in COPD-only) 14.

Taken together, all these findings suggest that reducing the number of medications may directly reduce the likelihood of $\mathrm{CRN}$, and indirectly alleviate the high burden of OOP prescription cost among older adults with ACO. As documented in published literature, clinicians and pharmacists can play an important role in deprescribing unnecessary medications. For example, during annual wellness visits, now covered by Medicare, a comprehensive review of medications can be performed and highlight opportunities for prescription regimen optimization113. Providers can proactively participate in deprescribing by: 1) discontinuing unnecessary therapy, 2) discontinuing duplicate therapies, 3) combining therapies as appropriate (i.e. combination inhalers), and 4) replacing high-cost therapies with more affordable options. In a survey of elderly Medicare beneficiaries, more than $30 \%$ of individuals reported they did not have the chance of talking with their provider about their medications in the last 12 months114. Intensive, chronic care management can be optimized through team-based care.

Another possible strategy to reduce number of medications is using non-pharmacological approaches such as lifestyle modification instead of prescribing medication. The Trial of Nonpharmacologic Interventions in the Elderly demonstrated that reduced sodium intake and weight loss could help discontinuation of antihypertensive medications in almost $40 \%$ of the intervention group 115,116. In addition to previous strategies, "time to benefit" (TTB) in relation to medication prescribing for older patients with multi-comorbidities can be utilized 117. TTB is a 
measurement of the time to significant observable benefit in randomized clinical trials of a medication compared with controls. This type of information is not routinely available. In the future, this may help guide clinicians to discontinue some medication prescriptions for older individuals when up-titration of a regimen was quicker than TTB.

\section{Strengths and Limitations}

To date, ours is the first study to examine the association between ACO and CRN among a nationally representative sample of fee-for-service community-dwelling Medicare beneficiaries. Medicare claims enabled us to identify current asthma and COPD status. Survey data provided the opportunity to control for a comprehensive set of covariates that are otherwise not available in claims data.

This study also has some limitations. Many variables in this study including CRN are self-reported, which may be subject to recall bias and social desirability bias. The findings of this study are not generalizable to all elderly Medicare beneficiaries as Medicare HMO enrollees or institutionalized beneficiaries were not included in this study. Despite the benefits of linked survey modules with claims, information about the age of onset, and the severity of ACO, asthma, COPD were not available in the dataset. Another limitation of this study is that CRN measures are for all types of medications and not for a specific medication group (e.g. inhalers). Finally, the study design is a cross-sectional study which makes it hard to establish a cause and effect relationship. Future cohort studies may help to evaluate the association between ACO and CRN.

\subsection{Conclusion}

CRN remains an issue among elderly Medicare beneficiaries. Individuals with ACO are vulnerable for any $\mathrm{CRN}$ and its specific forms. The high number of medications used among 
ACO individuals explained the association of ACO to CRN. Interventions which target reducing therapy burden and cost are needed to reduce CRN and prevent downstream effects of medication nonadherence in patients with ACO. 
Table 3.1

Unweighted $\mathbf{N}$ and Weighted Percentages of Characteristics by Asthma and Chronic Obstructive Pulmonary Disease (COPD) Status (Column \%)

Older (age $\geq 65$ years) Medicare Beneficiaries

Medicare Current Beneficiary Survey, 2006-2013

\begin{tabular}{|c|c|c|c|c|c|c|c|c|c|}
\hline & \multicolumn{2}{|c|}{$\begin{array}{c}\text { Asthma- } \\
\text { COPD } \\
\text { Overlap }\end{array}$} & \multicolumn{2}{|c|}{ Asthma-Only } & \multicolumn{2}{|c|}{ COPD-Only } & \multicolumn{2}{|c|}{$\begin{array}{l}\text { Neither-Asthma } \\
\text { Nor-COPD }\end{array}$} & \multirow[t]{2}{*}{ Sig } \\
\hline & $\mathbf{N}$ & Wt. \% & $\mathbf{N}$ & Wt. \% & $\mathbf{N}$ & Wt. \% & $\mathbf{N}$ & Wt. \% & \\
\hline ALL & 576 & 1.5 & 849 & 2.3 & 3,446 & 8.8 & 32,700 & 87.4 & \\
\hline Sex & & & & & & & & & **** \\
\hline Female & 382 & 67.4 & 650 & 77.7 & 1,686 & 49.7 & 18,468 & 56.1 & \\
\hline Male & 194 & 32.6 & 199 & 22.3 & 1,760 & 50.3 & 14,232 & 43.9 & \\
\hline Age in Years & & & & & & & & & $* * *$ \\
\hline $65-74$ & 205 & 40.3 & 391 & 53.5 & 1,199 & 41.2 & 13,098 & 48.0 & \\
\hline $75-84$ & 261 & 43.5 & 318 & 33.9 & 1,605 & 43.6 & 13,263 & 36.9 & \\
\hline 85 and above & 110 & 16.1 & 140 & 12.6 & 642 & 15.2 & 6,339 & 15.1 & \\
\hline Race/Ethnicity & & & & & & & & & $* * *$ \\
\hline White & 444 & 77.2 & 655 & 78.6 & 2,982 & 86.7 & 27,049 & 82.7 & \\
\hline Black & 46 & 8.0 & 84 & 9.3 & 164 & 4.7 & 2,269 & 6.8 & \\
\hline Latino & 48 & 7.8 & 59 & 6.5 & 142 & 3.9 & 1,863 & 5.8 & \\
\hline Other race & 37 & 7.0 & 51 & 5.7 & 151 & 4.7 & 1,449 & 4.7 & \\
\hline Marital Status & & & & & & & & & $* * *$ \\
\hline Married & 220 & 39.5 & 401 & 48.1 & 1,658 & 49.3 & 17,228 & 55.2 & \\
\hline Not married & 355 & 60.5 & 447 & 51.9 & 1,786 & 50.7 & 15,462 & 44.8 & \\
\hline Education & & & & & & & & & $* * *$ \\
\hline Less than high school & 191 & 31.7 & 174 & 19.1 & 1,131 & 31.8 & 7,649 & 21.3 & \\
\hline High school & 211 & 37.4 & 286 & 31.8 & 1,264 & 36.7 & 11,812 & 35.6 & \\
\hline Above high school & 172 & 30.8 & 387 & 49.2 & 1,040 & 31.5 & 13,112 & 43.1 & \\
\hline Poverty Status & & & & & & & & & $* * *$ \\
\hline Less than $200 \%$ FPL & 357 & 59.8 & 411 & 45.1 & 2,036 & 57.7 & 15,316 & 44.2 & \\
\hline At least $200 \%$ FPL & 219 & 40.2 & 438 & 54.9 & 1,410 & 42.3 & 17,384 & 55.8 & \\
\hline Prescription Coverage & & & & & & & & & $* * *$ \\
\hline Yes & 405 & 70.7 & 582 & 66.8 & 2,250 & 65.2 & 20,143 & 60.9 & \\
\hline No & 171 & 29.3 & 267 & 33.2 & 1,196 & 34.8 & 12,557 & 39.1 & \\
\hline High Burden of OOP Prescription Cost & & & & & & & & & $* * *$ \\
\hline Yes & 55 & 9.3 & 55 & 6.2 & 320 & 9.0 & 1,375 & 4.0 & \\
\hline No & 521 & 90.7 & 794 & 93.8 & 3,126 & 91.0 & 31,325 & 96.0 & \\
\hline High Burden of OOP Medical Cost & & & & & & & & & $* * *$ \\
\hline Yes & 171 & 28.3 & 149 & 17.1 & 740 & 20.9 & 3,825 & 11.0 & \\
\hline No & 405 & 71.7 & 700 & 82.9 & 2,706 & 79.1 & 28,875 & 89.0 & \\
\hline Number of Other Chronic Conditions & & & & & & & & & $* * *$ \\
\hline $0-1$ & 53 & 9.0 & 93 & 11.0 & 419 & 13.0 & 7,122 & 23.6 & \\
\hline $2-3$ & 273 & 47.6 & 471 & 56.4 & 1,754 & 50.7 & 17,539 & 53.4 & \\
\hline $4-6$ & 250 & 43.3 & 285 & 32.6 & 1,272 & 36.3 & 8,035 & 23.0 & \\
\hline Number of Medications & $\begin{array}{r}16.2 \\
1 \dagger\end{array}$ & $0.34 *$ & $12.74 \dagger$ & 0.29 & $12.51 \dagger$ & 0.17 ; & $7.37 \dagger$ & 0.06 & $* * *$ \\
\hline Calendar Year & & & & & & & & & \\
\hline 2006 & 86 & 13.2 & 122 & 12.7 & 504 & 13.2 & 4,982 & 13.2 & \\
\hline 2007 & 72 & 10.6 & 120 & 13.0 & 493 & 12.6 & 4,636 & 12.3 & \\
\hline 2008 & 81 & 12.2 & 97 & 9.9 & 460 & 11.8 & 4,266 & 11.7 & \\
\hline 2009 & 64 & 11.1 & 93 & 11.0 & 407 & 11.9 & 3,701 & 11.4 & \\
\hline 2010 & 56 & 10.6 & 94 & 12.4 & 392 & 12.4 & 3,459 & 11.3 & \\
\hline 2011 & 82 & 15.5 & 115 & 14.1 & 404 & 13.2 & 3,980 & 13.4 & \\
\hline 2012 & 66 & 12.3 & 109 & 13.8 & 406 & 12.5 & 3,965 & 13.3 & \\
\hline 2013 & 69 & 14.6 & 99 & 13.3 & 380 & 12.6 & 3,711 & 13.3 & \\
\hline
\end{tabular}

Note: Based on 37,571 person-years of community-dwelling elderly (age > 65 years) Medicare beneficiaries, continuously enrolled in fee-for-service Part A and Part B during the calendar year and alive during the calendar year, and had information in both cost and use and access to care files.

Significant group differences in asthma-COPD status were based on Rao-Scott chi-square tests. ANOVA test was used for number of unique medications.

FPL: federal poverty level; N: unweighted number; Sig: significance; Wt: weighted; $\uparrow:$ Mean; $\uparrow$ : Standard Error; $* * * p<.001$ 
Table 3.2

Unadjusted Odds Ratios (UOR), Adjusted Odds Ratios (AOR) and 95\% Confidence Intervals from

Separate Logistic Regressions on Any Cost-related Medication Nonadherence

Older (age $\geq 65$ years) Medicare Beneficiaries

Medicare Current Beneficiary Survey, 2006-2013

\begin{tabular}{|c|c|c|c|c|c|c|c|c|c|}
\hline & \multicolumn{3}{|c|}{ Ref $=$ No Asthma No COPD } & \multicolumn{3}{|c|}{ Ref $=$ Asthma Only } & \multicolumn{3}{|c|}{ Ref= COPD Only } \\
\hline & UOR & $95 \% \mathrm{CI}$ & Sig & UOR & $95 \% \mathrm{CI}$ & Sig & UOR & $95 \% \mathrm{CI}$ & Sig \\
\hline \multicolumn{10}{|l|}{ Unadjusted Models } \\
\hline $\begin{array}{l}\text { Asthma COPD } \\
\text { Overlap }\end{array}$ & 1.89 & {$[1.45,2.45]$} & $* * *$ & 1.11 & {$[0.78,1.59]$} & & 1.30 & {$[0.96,1.76]$} & \\
\hline Asthma Only & 1.69 & {$[1.34,2.14]$} & $* * *$ & Ref & & & 1.17 & {$[0.89,1.53]$} & \\
\hline COPD only & 1.45 & {$[1.26,1.67]$} & $* * *$ & 0.86 & {$[0.65,1.13]$} & & Ref & & \\
\hline \multirow[t]{2}{*}{ No Asthma No COPD } & Ref & & & 0.59 & {$[0.47,0.75]$} & $* * *$ & 0.69 & {$[0.60,0.79]$} & $* * *$ \\
\hline & AOR & $95 \% \mathrm{CI}$ & Sig & AOR & $95 \% \mathrm{CI}$ & Sig & AOR & $95 \% \mathrm{CI}$ & Sig \\
\hline \multicolumn{10}{|c|}{ Adjusted Models with All Factors except Number of unique medications } \\
\hline $\begin{array}{l}\text { Asthma COPD } \\
\text { Overlap }\end{array}$ & 1.50 & {$[1.14,1.96]$} & $* *$ & 1.08 & {$[0.76,1.54]$} & & 1.19 & {$[0.88,1.61]$} & \\
\hline Asthma Only & 1.38 & {$[1.09,1.75]$} & $* *$ & Ref & & & 1.10 & {$[0.84,1.43]$} & \\
\hline COPD only & 1.26 & {$[1.09,1.45]$} & $* *$ & 0.91 & {$[0.70,1.18]$} & & Ref & & \\
\hline No Asthma No COPD & Ref & & & 0.72 & {$[0.57,0.91]$} & $* *$ & 0.79 & {$[0.69,0.92]$} & $* *$ \\
\hline \multicolumn{10}{|l|}{ Fully Adjusted Models } \\
\hline $\begin{array}{l}\text { Asthma COPD } \\
\text { Overlap }\end{array}$ & 1.23 & {$[0.92,1.63]$} & & 1.00 & {$[0.70,1.44]$} & & 1.10 & {$[0.80,1.50]$} & \\
\hline Asthma Only & 1.22 & {$[0.97,1.54]$} & & Ref & & & 1.10 & {$[0.84,1.43]$} & \\
\hline COPD only & 1.12 & {$[0.96,1.30]$} & & 0.91 & {$[0.70,1.19]$} & & Ref & & \\
\hline No Asthma No COPD & Ref & & & 0.81 & {$[0.65,1.02]$} & & 0.90 & {$[0.77,1.04]$} & \\
\hline
\end{tabular}

Note: Based on 37,571 person-years of community-dwelling elderly (age $\geq 65$ years) Medicare beneficiaries, continuously enrolled in fee-for-service Part A and Part B during the calendar year and alive during the calendar year, and had information in both cost and use and access to care files.

COPD: Chronic Obstructive Pulmonary Disease; CRN: Cost-related Medication Non-Adherence; Ref: reference group; Sig:

significance

$* * * \mathrm{p}<.001 ; * * .001 \leq \mathrm{p}<.01 ; * .01 \leq \mathrm{p}<.05$ 
Table 3.3

Unadjusted Odds Ratios (UOR), Adjusted Odds Ratios (AOR) and 95\% Confidence Intervals from

Separate Logistic Regressions on Specific Forms of CRN

Older (age $\geq 65$ years) Medicare Beneficiaries

Medicare Current Beneficiary Survey, 2006-2013

\begin{tabular}{|c|c|c|c|c|c|c|c|c|c|}
\hline & \multicolumn{3}{|c|}{ Unadjusted Models } & \multicolumn{3}{|c|}{$\begin{array}{c}\text { Fully Adjusted Models } \\
\text { without Number of unique } \\
\text { medications } \\
\end{array}$} & \multicolumn{3}{|c|}{ Fully Adjusted Models } \\
\hline & UOR & $95 \% \mathrm{CI}$ & Sig & $\begin{array}{c}\text { AO } \\
\mathbf{R}\end{array}$ & $95 \% \mathrm{CI}$ & Sig & $\begin{array}{c}\text { AO } \\
\mathbf{R}\end{array}$ & $95 \% \mathrm{CI}$ & Sig \\
\hline \multicolumn{10}{|l|}{ CRN by Using Smaller Doses } \\
\hline $\begin{array}{l}\text { Asthma COPD } \\
\text { Overlap }\end{array}$ & 1.54 & {$[0.98,2.43]$} & & 1.21 & {$[0.75,1.94]$} & & 0.97 & {$[0.60,1.58]$} & \\
\hline Asthma Only & 1.72 & {$[1.16,2.55]$} & $* *$ & 1.42 & {$[0.95,2.10]$} & & 1.24 & {$[0.83,1.84]$} & \\
\hline COPD only & 1.49 & {$[1.23,1.82]$} & $* * *$ & 1.25 & {$[1.02,1.52]$} & * & 1.10 & {$[0.89,1.37]$} & \\
\hline \multicolumn{10}{|l|}{ No asthma No COPD } \\
\hline \multicolumn{10}{|c|}{ CRN by Skipping Medication Doses } \\
\hline $\begin{array}{l}\text { Asthma COPD } \\
\text { Overlap }\end{array}$ & 2.16 & {$[1.41,3.31]$} & $* * *$ & 1.58 & {$[1.00,2.50]$} & * & 1.36 & {$[0.87,2.11]$} & \\
\hline Asthma Only & 1.62 & {$[1.09,2.40]$} & $*$ & 1.28 & {$[0.87,1.87]$} & & 1.16 & {$[0.79,1.70]$} & \\
\hline $\begin{array}{l}\text { COPD only } \\
\text { No asthma and No } \\
\text { COPD } \\
\end{array}$ & 1.59 & {$[1.32,1.91]$} & $* * *$ & 1.31 & {$[1.09,1.58]$} & $* *$ & 1.20 & {$[1.00,1.46]$} & \\
\hline \multicolumn{10}{|c|}{ CRN by Failing to Fill Prescription } \\
\hline $\begin{array}{l}\text { Asthma COPD } \\
\text { Overlap }\end{array}$ & 2.25 & {$[1.59,3.17]$} & $* * *$ & 1.75 & {$[1.24,2.47]$} & $* *$ & 1.43 & {$[0.99,2.07]$} & \\
\hline Asthma Only & 1.61 & {$[1.20,2.15]$} & $* *$ & 1.30 & {$[0.97,1.73]$} & & 1.15 & {$[0.87,1.52]$} & \\
\hline COPD only & 1.65 & {$[1.42,1.92]$} & $* * *$ & 1.41 & {$[1.20,1.66]$} & $* * *$ & 1.25 & {$[1.05,1.49]$} & $*$ \\
\hline No asthma No COPD & & & & & & & & & \\
\hline \multicolumn{10}{|c|}{ CRN by Delaying Filling Prescription } \\
\hline $\begin{array}{l}\text { Asthma COPD } \\
\text { Overlap }\end{array}$ & 2.07 & {$[1.47,2.93]$} & $* * *$ & 1.48 & {$[1.02,2.14]$} & * & 1.26 & {$[0.87,1.81]$} & \\
\hline Asthma Only & 1.79 & {$[1.30,2.46]$} & $* * *$ & 1.37 & {$[0.99,1.90]$} & & 1.24 & {$[0.89,1.71]$} & \\
\hline COPD only & 1.69 & {$[1.37,2.09]$} & $* * *$ & 1.41 & {$[1.14,1.74]$} & $* *$ & 1.28 & {$[1.02,1.60]$} & $*$ \\
\hline No Asthma and COPD & & & & & & & & & \\
\hline
\end{tabular}

Note: Based on 37,571 person-years of community-dwelling elderly (age $\geq 65$ years) Medicare beneficiaries, continuously enrolled in fee-for-service Part A and Part B during the calendar year and alive during the calendar year, and had information in both cost and use and access to care files.

CI: Confidence Interval; COPD: Chronic Obstructive Pulmonary Disease; CRN: Cost-related Medication Non-Adherence; Sig: significance

$* * * \mathrm{p}<.001 ; * * .001 \leq \mathrm{p}<.01 ; * .01 \leq \mathrm{p}<.05$ 
Figure 3.1

Prevalence of any CRN and Specific Types of CRN

By Asthma-COPD Status (i.e., Asthma-COPD Overlap, Asthma-Only, COPD-Only, Neither Asthma nor COPD)

Older (age $\geq 65$ years) Medicare Beneficiaries

Medicare Current Beneficiary Survey, 2006-2013

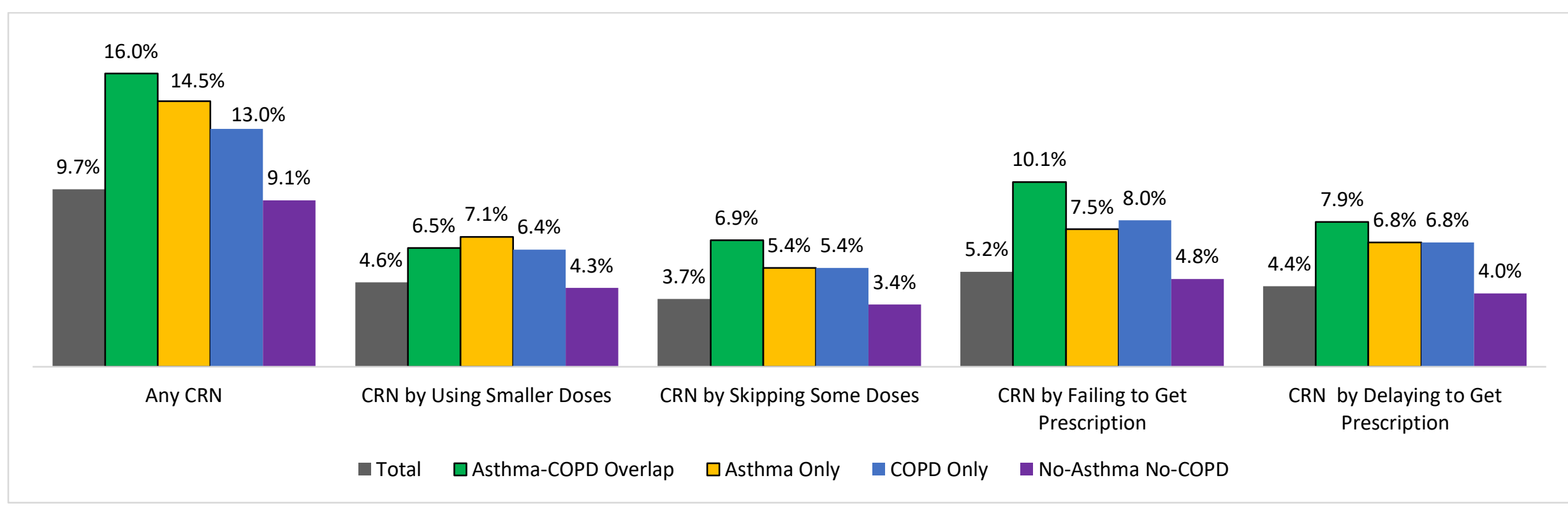

Note: Based on 37,571 person-years of community-dwelling elderly (age $\geq 65$ years) Medicare beneficiaries, continuously enrolled in fee-for-service Part A and Part B during the calendar 


\section{CHAPTER 4}

\section{Impact of Initial Maintenance Therapy Type on Medication Adherence Trajectories among Older Adults with Asthma and COPD Overlap}

\subsection{Abstract}

Objective: To examine the impact of initial maintenance therapy (IMT) type (inhaled corticosteroids (ICS) vs. fixed dose combinations of ICS and long acting beta agonists (ICS/LABA)) on trajectories of adherence among Asthma and Chronic Obstructive Pulmonary Disease overlap (ACO) among older adults ( $\geq 65$ years).

Methods: This study used a retrospective cohort of older adults with ACO using longitudinal data from a 10\% sample of Optum's Deidentified Clinformatics ${ }^{\circledR}$ Data Mart. We adopted group-based trajectory modeling to identify medication adherence trajectories over 12 months. Multinomial logistic regressions were used to evaluate the unadjusted and adjusted association of IMT medication and adherence trajectory categories. All analyses accounted for treatment option selection bias with inverse probability treatment weighting.

Results: Of 1,555 individuals, $73 \%$ of the sample used ICS/LABA for IMT. Four medication adherence trajectories were observed regardless of regimen: 1) persistent high adherence $(12.0 \%) ; 2)$ progression to high adherence $(20.8 \%) ; 3)$ progression to low adherence $(10.5 \%)$ and 4) persistent low adherence (56.7\%). Those who were initiated on ICS/LABA were less likely to have "persistent low adherence" (Unadjusted Odds Ratio $=0.44,95 \%$ Confidence Interval $(\mathrm{CI})=$ $[0.29,0.67])$ as compared to those with ICS monotherapy when "persistent high adherence" was used as the reference group. The relationship remained significant in adjusted regressions (Adjusted Odds Ratio $=0.38,95 \% \mathrm{CI}=[0.24,0.59]$ ). 
Conclusions: Real-world evidence suggests that using ICS/LABA for IMT may decrease the likelihood of "persistent" low adherence over time among older adults with ACO compared to ICS monotherapy.

\subsection{Introduction}

Many adults have co-existing asthma and Chronic Obstructive Pulmonary Disease (COPD) known as "Asthma-COPD overlap" (ACO)9. Treatment for ACO requires treating both the underlying asthma and COPD by using maintenance therapy with inhaled medications. The joint guidelines of Global Initiative for Asthma (GINA) and Global Initiative for Chronic Obstructive Lung Disease (GOLD) recommend inhaled corticosteroids (ICS) with or without a long-acting beta-agonist (LABA) for initial maintenance therapy (IMT) of individuals with $\mathrm{ACO}_{7,3}$.

Compared to ICS monotherapy, use of ICS/LABA for IMT of ACO has some advantages. First, ICS/LABA can reduce both the inflammatory nature of asthma and the obstructive nature of COPD 19. Second, prior studies indicate using ICS/LABA may improve medication adherence compared to ICS. Although the association of using ICS/LABA and adherence to IMT has not been directly studied among patients with ACO, these findings have been noted among patients with asthma48,118-121. One possible reason is that the use of LABA in fixed-dose combination with ICS has demonstrated benefit in reducing symptoms and exacerbations quickly compared to ICS monotherapy which may increase the medication value to the patient122,123. Finally, one observational study reported that ICS/LABA reduced the risk of myocardial infarction, hospitalization, and death among patients with ACO, as compared to ICS monotherapy47.

To date, no study has evaluated adherence to IMT or the effect of IMT type on medication adherence among older adults with ACO. However, this is a crucial topic to better understand because older adults are the most vulnerable group of individuals to experience poor adherence 
and subsequent negative outcomes. Older adults (age > 70 years) often have higher prevalence of poor medication adherence, compared to younger adults (age $\leq 50$ years), due to regimen complexity, polypharmacy, prescription costs, and multiple comorbidities 124,125. For example, prior studies have suggested that $70 \%$ of COPD patients 30 and nearly $50 \%$ of adults with asthma27 have poor adherence to IMT, suggesting that such patterns may also be present in older adults with ACO. Poor medication adherence can amplify the negative health effects of older adults with ACO. Among patients with asthma or COPD, poor adherence to maintenance therapy is associated with increased risk of adverse events such as hospitalization, disease burden, and mortality. 126-128 Therefore, older individuals with ACO may also experience these negative consequences, perhaps to an even greater degree.

It is important to analyze medication adherence over time because static adherence metrics (e.g. proportion of days covered (PDC) or medication possession ratio (MPR)) average adherence over time and ignore "within" individuals' differences in adherence over time.129 For example, an individual who uses medications for the first six months and discontinues for the following six months will have the same PDC as an individual who uses the prescription every other month. As medication adherence is known to vary over time among adults with chronic lung diseases31, examination of adherence trajectories over time is important. Such information can precisely locate the time point at which adherence changes. Furthermore, adherence trajectories can guide development of customized intervention efforts at the right time 130.

Therefore, the current study used group-based trajectory modeling (GBTM) of adherence to IMT over time and analyzed the association of IMT type to trajectory membership among older adults using claims data from Optum Clinformatics ® DataMart. Although, GBTM method has been previously used for studying patterns of medication adherence to IMT among patients 
with asthma25, this study is the first application of the GBTM approach for examining individuals with ACO.

\subsection{Methods}

\section{Data Source}

This study used health insurance claims from a 10\% sample of Optum Clinformatics $®$ DataMart from 1/1/2007 through 6/30/2017. This database contains information on prescription claims [e.g. NDC codes, generic drug names, prescription fill dates, days supply, and costs of medications], inpatient and outpatient medical claims [e.g. International Classification of Diseases (ICD) 9 th and 10th revision diagnoses, date of service, and costs of services], and eligibility information [e.g. sex, date of birth, plan type, and insurance type].

\section{Study Design}

We used a longitudinal retrospective cohort design with a 24-month observation period (12-month baseline and 12-month follow-up periods). We anchored the baseline period and the follow-up period to the index date (i.e. first observed ICS or ICS/LABA fill date between 10/1/2008 to 9/30/2016). Baseline consisted of 12 months before the index date. The baseline period was used to identify ACO and other characteristics. In the 12-months follow up period, we measured medication adherence every month. Thus, each person had 12 observations (T1 to T12).

\section{Study Cohort: Older Adults with ACO}

Only individuals with ACO diagnosis were included in this study. The first step involved the identification of patients with either asthma or COPD during the baseline period. We identified asthma or COPD by using the International Classification of Diseases, Clinical Modification (ICD) 9 or ICD10 codes (Appendix 7.2). These diagnosis codes were derived from 
published research73,74. Individuals were required to have at least one inpatient visit or two outpatient visits (at least 14 days apart) with either asthma or COPD codes. Individuals with both asthma and COPD diagnoses during the baseline were identified as indivduals with ACO.

The study cohort included only older (age $\geq 65$ years) Medicare advantage beneficiaries who initiated maintenance therapy with ICS monotherapy or ICS/LABA from 10/1/2008 to 9/30/2016. These patients were required to have continuous enrollment in both the baseline and follow-up periods. We excluded individuals who used any other maintenance therapy (i.e. ICS, LABA, long acting muscarinic antagonist (LAMA), and their combination forms) during the 12month pre-index period (Appendix 7.9).

\section{Measures}

Dependent Variables: Medication Adherence and Medication Adherence Trajectory Categories derived from GBTM

We measured adherence to ICS or ICS/LABA for every 30-days during the follow-up period. We created binary variables (yes/no) for each month based on the days supply for each month after the index date25. These 12 dichotomized monthly indicators were used in a GBTM. As described below, the GBTM model yielded four medication adherence patterns: (1) persistent high adherence; (2) progression to high adherence; (3) progression to low adherence; and (4) persistent low adherence. These categories were used as the dependent variable to analyze the association of IMT type to medication adherence.

Key Independent Variable: Type of IMT (ICS and ICS/LABA)

Two usual treatment options for IMT were ICS monotherapy and fixed-dose combination of ICS/LABA. National drug codes were used to identify these medications. To ensure that individuals used ICS or ICS/LABA as IMT, we defined a look-back period of 365 days and only 
individuals who did not have any ICS, ICS/LABA, LABA, or LAMA use were considered as initiating maintenance therapy.

\section{Other Independent Variables}

Selection of covariates was guided by the Anderson Health behavior model50. Per the framework, adherence to IMT may be affected by: (1) pre-disposing variables (sex [male/female], age); (2) enabling factors (insurance plan [health maintenance organization (HMO)/Non-HMO], annual out-of-pocket prescription costs); (3) need factors (dementia [yes/no], depression [yes/no], anxiety [yes/no], diabetes [yes/no], heart diseases (the presence of at least one of the following: chronic heart failure, coronary artery disease, or arrhythmia) [yes/no], hypertension [yes/no], the number of other chronic conditions (cancer, arthritis, hyperlipidemia, hepatitis, HIV, osteoporosis, chronic kidney disease, stroke), the number of short acting beta agonists (SABA), the number of courses of oral corticosteroids or antibiotics, polypharmacy [concomitant use of five or more classes of medications within a 90 day period before the index date]); (4) personal health practices (obesity diagnosis [yes/no] and tobacco use diagnosis [yes/no]; (5) external factors (region [Northeast/Midwest/ South/West]).

\section{Statistical Analyses}

Accounting for Selection Bias of Treatment:

We observed significant differences in baseline characteristics by IMT type. Specifically, individuals using ICS/LABA were more likely to be male, enrolled in non-HMO plans, to be prescribed a SABA, more likely to live in the Southern region of the US, more likely to have clinically recorded tobacco use, and less likely to have diagnosed anxiety, as compared to ICS monotherapy. To control for this observed selection bias, we derived inverse probability of treatment weights (IPTW). IPTWs were estimated from a logistic regression in which IMT 
type (ICS vs ICS/LABA) was the dependent variable. The covariates included sex, age, being enrolled in an HMO plan, out-of-pocket prescription costs, presence of diabetes, anxiety, depression or heart diseases, number of SABA, tobacco use diagnosis, the number of courses of antibiotics, and region of residency. IPTWs were then used in the GBTM of medication adherence. Once the medication adherence patterns were identified, we compared the unadjusted and adjusted associations of IMT type to the trajectory groups using the IPTW-adjusted multinomial logistic regressions.

Identification of Medication Adherence Trajectory Groups:

We identified clusters of patients with similar adherence patterns using the GBTM method. GBTM uses the maximum likelihood estimation to identify changes in medication adherence patterns over time. 131,132 GBTM uses the data to empirically group individuals. Under this method, patterns of adherence are identified based on linear and non-linear specifications and the probabilities of each individual belonging to these trajectories are calculated. Individuals are assigned to the group for which they have the highest probability. We used different specifications to identify these groups by using either cubic, quadratic, or quartic terms 133,134. Evaluating Medication Adherence by Using Generalized Estimating equations:

In addition to GBTM, we also used unadjusted and adjusted generalized estimating equations (GEE) to evaluate the association of IMT type to medication adherence over time. We used population-averaged GEE for repeated measures. Results from the GEE analyses are summarized in Appendix 7.10. All data management and analyses were conducted with SAS 9.4 (SAS Institute Inc., Cary, NC) and Stata 14 (Stata Corp LLC, College Station, TX) was used for GBTM and GEE modeling. 


\subsection{Results}

\section{Sample Characteristics Baseline Characteristics}

In our study cohort of older adults with ACO and initiated on ICS or ICS/SABA as IMT $(\mathrm{N}=1,554)$, the majority were female $(66.6 \%)$; mean age was $75.2 \pm 6.1$ years. The majority of individuals were enrolled in an HMO plan (64.9\%). The baseline characteristics of our study cohort is provided in Appendix 7.11. An overwhelming majority (73.2\%) used ICS/LABA and $26.8 \%$ used ICS for IMT.

\section{IPTW-adjusted Baseline Characteristics by IMT Type}

The unweighted numbers and IPTW-adjusted weighted percentages baseline characteristics by IMT type are displayed in Table 1. As can be seen in Table 1, there were no statistically significant differences in baseline characteristics between the two groups after IPTW adjustments.

\section{Identification of Medication Adherence Trajectories with GBTM}

We initially specified eight groups with quadratic and higher-order polynomial specifications. After a preliminary examination of the results, we finalized trajectories to four groups based on the following criteria: 1) the Bayesian information criterion (BIC); 2) significant p-values (<.05) for the highest-order polynomial parameter; 3 ) at least $5 \%$ of the population assigned to each trajectory group; 4) no polynomial overfitting; 5) narrow confidence intervals; and 6) clinically meaningful grouping $131,135-137$. The final trajectory model passed all Nagin's diagnostic criteria of the "average of posterior probabilities (PP) $>0.7$ " and the "odds of correct classification $(\mathrm{OCC})>0.5$ " for all groups 131,136 .

As shown in Figure 1, we identified four different adherence trajectories. These trajectories can be described as: (1) persistent low adherence (56.7\%); (2) persistent high 
adherence (12.0\%), 3) progression to low adherence (10.5\%); and (4) progression to high adherence $(20.8 \%)$.

\section{Unadjusted and Adjusted Associations of IMT type to Adherence Trajectory Groups}

The four adherence trajectories were significantly different in terms of IMT type (Table 2) with a higher percentage of individuals who used ICS/LABA having "persistent high adherence", compared to those prescribed ICS monotherapy (82.9\% vs. 68.1). Unadjusted odds ratios (UOR), adjusted odds ratios (AOR), and 95\% Confidence intervals (CI) from the multinomial logistic regressions on medication adherence categories are summarized in Table 3. In the unadjusted multinomial logistic regression models, those who were initiated on ICS/LABA were 56\% less likely to have "persistent low adherence" (UOR $=0.44,95 \%$ CI $[0.29$, 0.67]), compared to those with ICS monotherapy when "persistent high adherence" was used as the reference group for the dependent variable. The relationship remained significant in adjusted regressions that controlled for only predisposing factors $(\mathrm{AOR}=0.44,95 \% \mathrm{CI}=[0.29,0.66])$ and remained significant in all subsequent models. In the fully adjusted model, those with ICS/LABA as IMT were less likely to have "persistent low adherence" $(\mathrm{AOR}=0.37,95 \% \mathrm{CI}=$ $[0.24,0.59])$.

\subsection{Discussion}

This study is the first study to report longitudinal patterns of adherence to IMT among older adults with ACO seeking care in real-world practices and who were initiated on ICS or ICS/LABA for maintenance therapy by using a GBTM approach. In our study cohort of older adults with ACO, ICS/LABA use was very common, with three in four older adults using combination therapy. In a study conducted on adults ( $\geq 18$ years) using 2008 and 2011 Truven Health Analytics MarketScan Commercial and Medicare Supplemental databases approximately 
$75 \%$ of adults with ACO and on maintenance therapy were prescribed ICS/LABA 74. The differences in the prevalence rate of ICS/LABA can be due to differences in study population, time period, and insurance coverage.

We identified four distinct patterns of medication adherence: 1) persistent low adherence; 2) persistent high adherence; 3) progression to low adherence and 4) progression to high adherence. Overall, the majority of older adults (57\%) had persistent poor adherence and only $11 \%$ had "persistent high adherence." As there are no published studies on medication adherence trajectories over time among older adults with ACO, we compared our results to studies that focused on adults with asthma or COPD. A recent study conducted in Australia reported that the "persistent low adherence" trajectory to IMT was observed among 58\% of individuals between 12 and 45 years old. Studies that use PDC for measuring medication adherence also reported the high prevalence of medication non-adherence ( $<80 \%$ PDC) among patients with asthma or COPD. For example, a systematic review of 39 clinical trials reported that only $47 \%$ to $57 \%$ of individuals with asthma were adherent to maintenance therapy27. Moreover, in a retrospective cohort study on Medicare beneficiaries with COPD, the average monthly adherence to IMT was plateaued at $35 \%$ by the seventh month 31 . Our study's findings demonstrating that the majority of older adults with ACO had persistent low adherence is concerning. This finding suggests that there is a significant room for improvement in adherence to achieve better disease management, improved patient safety, and reduced exposure to unnecessary treatment intensification.

The results of this study confirmed that the adherence to IMT varies over time. For example, one third of patients changed their adherence behavior after six months of starting IMT. This may indicate a heightened surveillance of adults who were initiated on IMT, and there may be a limited "window of opportunity" to deliver evidence-based interventions that improve 
adherence. Although there are many types of interventions to improve medication adherence among individuals with asthma or COPD, motivational interviewing 138 , coaching and patient education 139, shared decision-making 140 , and simplification of regimen 141 have been proven to improve adherence. Our study findings suggest that these interventions may need to be delivered at the right time to improve medication adherence.

Our study findings also suggest that the type of therapy (type of IMT) may be an important modifiable factor for reducing the risk of consistent low-adherence. In our study, older adults who were initiated on ICS/LABA were less likely to have "persistent low adherence". Previous studies among patients with asthma also support this finding. A clinical trial conducted by Perrin et al. reported that adherence to ICS/LABA was marginally higher, as compared to ICS monotherapy142. Similarly, four observational studies among patient with asthma concluded that that use of ICS/LABA is likely to improve adherence to IMT or maintenance therapy compared with ICS $48,118-121$. As stated in the introduction ICS/LABA confers many advantages. Although many patients perceive that inhalers are effective in relieving symptoms, this perceived efficacy of ICS/LABA may be higher. This higher perceived efficacy can be due to a faster improvement of symptoms by using ICS/LABA. A previous clinical trial among patients with asthma has reported that ICS/LABA resulted in a faster improvement in asthma symptoms compared to ICS monotherapy for IMT143. While simplifying a medication regimen is optimal for improved adherence, prescribers do not need to order two inhalers to achieve dual therapy; ICS/LABA are available in combination as one inhaler. Future randomized clinical trials are needed to investigate the differences in improvement of symptoms between ICS/LABA and ICS for IMT of ACO. Our study findings also suggest that therapy- 
related factor (type of IMT) may be an important modifiable factor in reducing the risk of consistent low-adherence.

\subsection{Limitations}

This study has some limitations. One limitation is the use of medication fills data for assessing adherence. In fact, adherence based on medication fills data assumes that filled medications are taken and used properly by patients. This assumption can result in overestimating adherence. As this study used retrospective cohort design using claims data, it has the inherent limitations of the observational study (under coding of diagnosis, lack of clinical markers of disease severity, and lack of information on behavioral variables). Other factors that may impact adherence to IMT (e.g. patient-physician communication, severity of symptoms, fear of side effects, and family support) were not adjusted, and this may change the impact of IMT type on adherence behavior.

\subsection{Conclusion}

Among older adults with ACO seeking care in real-world settings, we identified potential concern with high rates of non-adherence based on fill rates. Our study findings suggest that using ICS/LABA for IMT may reduce the risk of "persistent low adherence" over time among older adults with ACO. Future studies need to target older with consistently low adherence to maintenance therapy to explore drug-related and non-drug-related factors that are barriers or facilitators to adherence patterns over time. 
Table 4.1

Characteristics of Older Adults with Asthma-COPD Overlap

by Initial Maintenance Therapy Options

Inverse Probability Treatment Weighting

Optum Clinformatics 10\% Sample, 2007-2017

\begin{tabular}{|c|c|c|c|c|c|}
\hline & \multicolumn{2}{|c|}{ ICS/LABA } & \multicolumn{2}{|c|}{ ICS } & \multirow[b]{2}{*}{ Sig } \\
\hline & N/ Mean & Wt \% & $\mathbf{N}$ & Wt \% & \\
\hline $\mathbf{A L L}$ & 1137 & 73.2 & 417 & 26.8 & \\
\hline \multicolumn{5}{|l|}{ Sex } & \\
\hline Female & 757 & 66.6 & 276 & 66.4 & \\
\hline Male & 380 & 33.4 & 140 & 33.6 & \\
\hline Age (Mean, SD) & 75.01 & 6.06 & 75.75 & 6.27 & \\
\hline \multicolumn{5}{|l|}{ Insurance Plan } & \\
\hline $\mathrm{HMO}$ & 739 & 64.9 & 270 & 64.9 & \\
\hline Non- HMO & 399 & 35.1 & 146 & 35.1 & \\
\hline \multicolumn{6}{|l|}{ Out-of-pocket Payments for } \\
\hline Prescription Drugs (Mean, SD) & 533.22 & 739.23 & 552.41 & 750.56 & \\
\hline \multicolumn{6}{|l|}{ Depression } \\
\hline Yes & 145 & 12.8 & 52 & 12.4 & \\
\hline No & 992 & 87.2 & 365 & 87.6 & \\
\hline \multicolumn{6}{|l|}{ Anxiety } \\
\hline Yes & 120 & 10.5 & 44 & 10.5 & \\
\hline No & 1018 & 89.5 & 373 & 89.5 & \\
\hline \multicolumn{6}{|l|}{ Diabetes } \\
\hline Yes & 410 & 36.0 & 165 & 39.6 & \\
\hline No & 727 & 64.0 & 252 & 60.4 & \\
\hline \multicolumn{6}{|l|}{ Hypertension } \\
\hline Yes & 802 & 70.5 & 304 & 72.9 & \\
\hline No & 335 & 29.5 & 113 & 27.1 & \\
\hline \multicolumn{6}{|l|}{ Heart Diseases } \\
\hline Yes & 481 & 42.3 & 171 & 41.1 & \\
\hline No & 656 & 57.7 & 245 & 58.9 & \\
\hline \multicolumn{6}{|l|}{ \# Other Chronic Conditions } \\
\hline 0 & 218 & 19.2 & 70 & 16.7 & \\
\hline $1-2$ & 708 & 62.2 & 268 & 64.4 & \\
\hline $3-4$ & 211 & 18.6 & 79 & 18.9 & \\
\hline \# SABA (Mean, SD) & 1.75 & 2.77 & 1.78 & 2.73 & \\
\hline \multicolumn{6}{|l|}{ Polypharmacy } \\
\hline Yes & 794 & 69.8 & 303 & 72.7 & \\
\hline No & 343 & 30.2 & 114 & 27.3 & \\
\hline \multicolumn{6}{|l|}{ Obesity Diagnosis } \\
\hline Yes & 198 & 17.4 & 66 & 15.9 & \\
\hline No & 939 & 82.6 & 350 & 84.1 & \\
\hline \multicolumn{6}{|l|}{ Tobacco Use Diagnosis } \\
\hline Yes & 143 & 12.6 & 50 & 11.9 & \\
\hline No & 994 & 87.4 & 367 & 88.1 & \\
\hline \multicolumn{6}{|l|}{ Region } \\
\hline Northeast & 125 & 11.0 & 46 & 10.9 & \\
\hline Midwest & 200 & 17.6 & 74 & 17.7 & \\
\hline South & 442 & 38.9 & 161 & 38.8 & \\
\hline West & 369 & 32.5 & 136 & 32.6 & \\
\hline
\end{tabular}

Note: Based on 1,556 older adults aged 65 years or older with ACO diagnosis, initiated on maintenance therapy (either inhaled corticosteroid only or fixed dose combination of inhaled corticosteroids and-long-acting beta agonist) and continuously enrolled during the observation period.

Abbreviations: ACO: Asthma COPD Overlap; HMO: Health Maintenance Organization; SABA: Short-Acting Beta Agonist; SD: Standard deviation; Sig: Significance; Wt: Weight; \#: Number 
Table 4.2

Characteristics of Older Adults with Asthma-COPD Overlap

By Medication Adherence Trajectories

Initiated on Maintenance Therapy

Optum Clinformatics 10\% Sample, 2007-2017

\begin{tabular}{|c|c|c|c|c|c|c|c|c|c|}
\hline & \multicolumn{2}{|c|}{$\begin{array}{l}\text { Persistent Low } \\
\text { Adherence } \\
\end{array}$} & \multicolumn{2}{|c|}{$\begin{array}{l}\text { Progression to } \\
\text { Low Adherence }\end{array}$} & \multicolumn{2}{|c|}{$\begin{array}{l}\text { Progression to } \\
\text { High Adherence }\end{array}$} & \multicolumn{2}{|c|}{$\begin{array}{l}\text { Persistent High } \\
\text { Adherence }\end{array}$} & \multirow[t]{2}{*}{ Sig } \\
\hline & $\mathbf{N}$ & Wt \% & $\mathbf{N}$ & Wt \% & $\mathbf{N}$ & Wt \% & $\mathbf{N}$ & Wt \% & \\
\hline ALL & 891 & 57.3 & 322 & 20.7 & 166 & 10.7 & 177 & 11.4 & \\
\hline IMT Type & & & & & & & & & **** \\
\hline ICS/LABA & 607 & 68.1 & 257 & 79.8 & 128 & 77.1 & 145 & 82.9 & \\
\hline ICS & 284 & 31.9 & 65 & 20.2 & 38 & 22.9 & 30 & 17.1 & \\
\hline \multicolumn{10}{|l|}{ Sex } \\
\hline Female & 596 & 66.9 & 207 & 64.3 & 115 & 69.3 & 117 & 66.9 & \\
\hline Male & 295 & 33.1 & 115 & 35.7 & 51 & 30.7 & 58 & 33.1 & \\
\hline Age (Mean, SD) & 75.1 & 6.1 & 75.1 & 6.0 & 75.2 & 6.2 & 75.9 & 6.2 & *** \\
\hline \multicolumn{10}{|l|}{ Insurance Plan } \\
\hline HMO & 579 & 65.0 & 211 & 65.5 & 112 & 67.5 & 107 & 61.1 & \\
\hline Non- HMO & 312 & 35.0 & 111 & 34.5 & 54 & 32.5 & 68 & 38.9 & \\
\hline $\begin{array}{l}\text { OOP Prescription Costs } \\
\text { in hundreds (Mean (SD) }\end{array}$ & 5.3 & 7.0 & 5.9 & 9.1 & 6.1 & 7.7 & 3.9 & 4.8 & * \\
\hline \multicolumn{10}{|l|}{ Depression } \\
\hline Yes & 126 & 14.1 & 34 & 10.6 & 18 & 10.8 & 23 & 13.1 & \\
\hline No & 765 & 85.9 & 288 & 89.4 & 148 & 89.2 & 152 & 86.9 & \\
\hline Diabetes & & & & & & & & & ** \\
\hline Yes & 354 & 39.7 & 116 & 36.0 & 47 & 28.3 & 52 & 29.7 & \\
\hline No & 537 & 60.3 & 206 & 64.0 & 119 & 71.7 & 123 & 70.3 & \\
\hline Heart Diseases & & & & & & & & & + \\
\hline Yes & 398 & 44.7 & 130 & 40.4 & 58 & 34.9 & 66 & 37.7 & \\
\hline No & 493 & 55.3 & 192 & 59.6 & 108 & 65.1 & 109 & 62.3 & \\
\hline \multirow{2}{*}{\multicolumn{10}{|c|}{$\begin{array}{l}\text { \# Other Chronic } \\
\text { Conditions }\end{array}$}} \\
\hline & & & & & & & & & \\
\hline 0 & 163 & 18.3 & 58 & 18.0 & 30 & 18.1 & 35 & 20.0 & \\
\hline $1-2$ & 556 & 62.4 & 211 & 65.5 & 107 & 64.5 & 104 & 59.4 & \\
\hline 3 and more & 172 & 19.3 & 53 & 16.4 & 29 & 17.5 & 36 & 20.5 & \\
\hline \# SABA & & & & & & & & & **** \\
\hline 0 & 342 & 38.4 & 115 & 35.7 & 64 & 38.6 & 51 & 29.1 & \\
\hline $1-3$ & 455 & 51.1 & 151 & 46.9 & 72 & 43.4 & 81 & 46.3 & \\
\hline $4-6$ & 62 & 7.0 & 34 & 10.6 & 17 & 10.2 & 25 & 14.3 & \\
\hline$\geq 7$ & 32 & 3.6 & 22 & 6.8 & 13 & 7.8 & 18 & 10.3 & \\
\hline$\geq 2$ Courses of Antibiotics & & & & & & & & & * \\
\hline Yes & 469 & 52.6 & 148 & 46.0 & 78 & 47.0 & 72 & 41.1 & \\
\hline No & 422 & 47.4 & 174 & 54.0 & 88 & 53.0 & 103 & 58.9 & \\
\hline \multicolumn{10}{|l|}{ Polypharmacy } \\
\hline Yes & 628 & 70.5 & 225 & 69.9 & 120 & 72.3 & 124 & 70.9 & \\
\hline No & 263 & 29.5 & 97 & 30.1 & 46 & 27.7 & 51 & 29.1 & \\
\hline \multicolumn{10}{|l|}{ Obesity Diagnosis } \\
\hline Yes & 158 & 17.7 & 59 & 18.3 & 23 & 13.9 & 20 & 11.4 & \\
\hline No & 733 & 82.3 & 263 & 81.7 & 143 & 86.1 & 155 & 88.6 & \\
\hline \multicolumn{10}{|l|}{ Region } \\
\hline Northeast & 100 & 11.2 & 32 & 9.9 & 19 & 11.4 & 21 & 12.0 & \\
\hline Midwest & 133 & 14.9 & 71 & 22.0 & 31 & 18.7 & 38 & 21.7 & \\
\hline South & 363 & 40.7 & 117 & 36.3 & 67 & 40.4 & 58 & 33.1 & \\
\hline West & 295 & 33.1 & 102 & 31.7 & 49 & 29.5 & 58 & 33.1 & \\
\hline
\end{tabular}

Note: Based on 1,556 older adults aged 65 years or older with Asthma Chronic Obstructive Pulmonary Disease Overlap, initiated on maintenance therapy (either inhaled corticosteroid only or fixed dose combination of inhaled corticosteroids and-long-acting beta agonist) and continuously enrolled during the observation period. 
Abbreviations: ACO: Asthma COPD Overlap; HMO: Health Maintenance Organization; ICS: Inhaled Corticosteroid; ICS/LABA: Fixed dose combination of inhaled corticosteroid-and-long-acting beta agonist; IMT: Initial Maintenance Therapy; N: Number; OOP: Out of Pocket; SABA: Short-Acting Beta Agonist; SD: Standard deviation; Sig: Significance; Wt: Weight; \#: Number $* * * \mathrm{p}<.001, * * .001 \leq \mathrm{p}<.01, * .01 \leq \mathrm{p}<.005,+.05 \leq \mathrm{p}<.1$ 
Table 4.3

Unadjusted Odds Ratios (UOR), Adjusted Odds Ratios (AOR) and 95\% Confidence Intervals from

Multinomial Logistic Regressions on Medication Adherence Trajectory Groups

with Persistent High Adherence as Reference Group

Inverse Probability Treatment Weighting

among Older Medicare Beneficiaries with ACO and Initiated on Maintenance Therapy

Optum Clinformatics 10\% Sample, 2007-2017

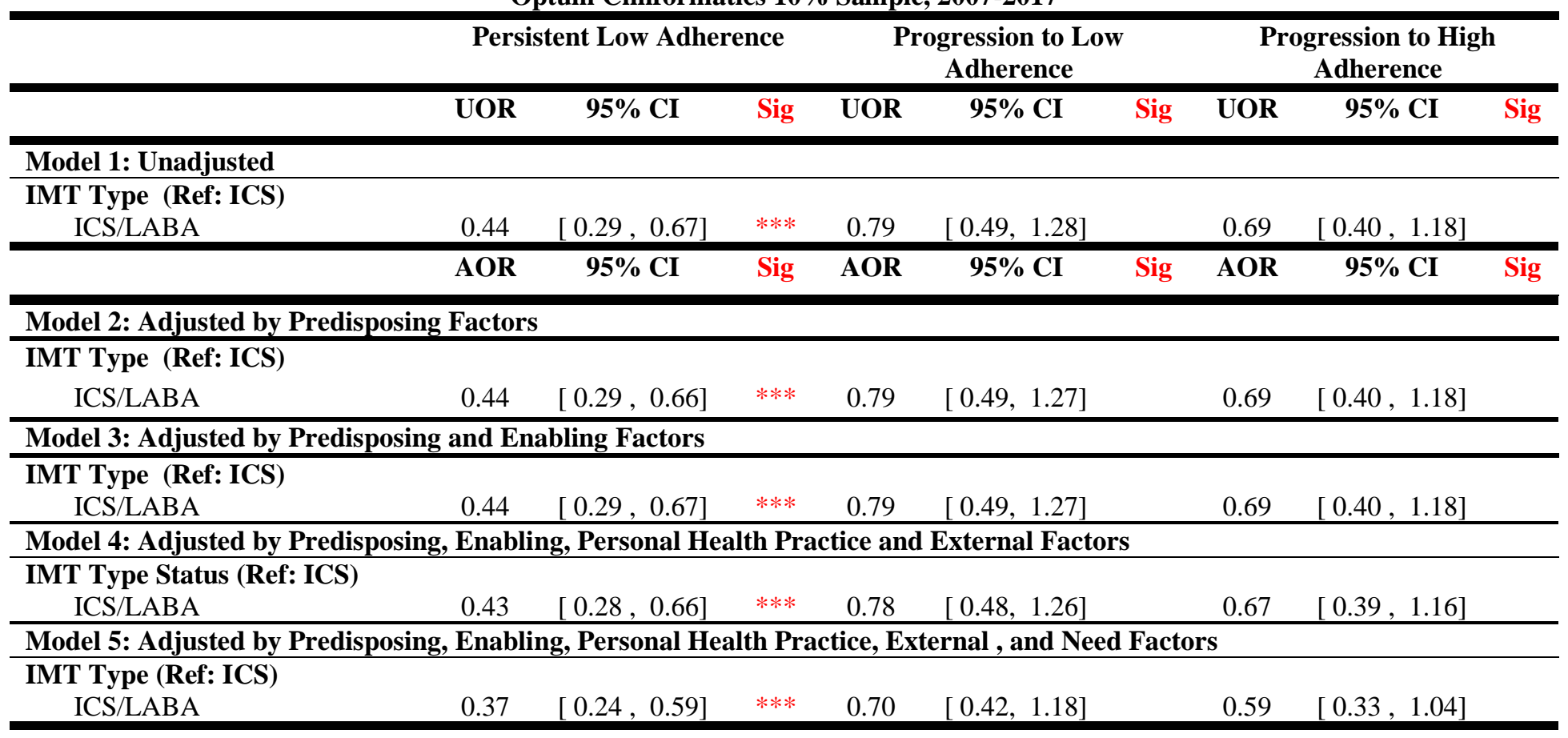

Note: Based on 1,556 older adults aged 65 years or older with Asthma Chronic Obstructive Pulmonary Disease overlap, initiated on maintenance therapy (either inhaled corticosteroid only or fixed dose combination of inhaled corticosteroids and-long-acting beta agonist) and continuously enrolled during the observation period.

Pre-disposing factors were sex, and age. Enabling factors consisted of insurance plan type, annual out-of-pocket payments for prescription drugs. Personal health practices were: Obesity diagnosis and tobacco use recorded in claims. External environmental factors included region of residency. Need factors were: dementia, depression, anxiety, diabetes, heart diseases, hypertension, the number of other chronic conditions, the number of SABA, the number of courses of oral corticosteroids, the number of courses of antibiotics, and polypharmacy.

Abbreviations: ACO: Asthma Chronic Obstructive Pulmonary Disease Overlap; ICS: Inhaled Corticosteroid; ICS/LABA: Fixed dose combination of inhaled corticosteroid-and-long-acting beta agonist; ref: reference group; SABA: Short-Acting Beta Agonist; Sig:

Significance

$* * * \mathrm{p}<.001$ 
Figure 4.1

Trajectories of Adherence to Initial Maintenance Therapy Among Older ( $\geq 65$ years old) Adults with Asthma-COPD Overlap

Optum Clinformatics 10\% Sample, 2007-2017

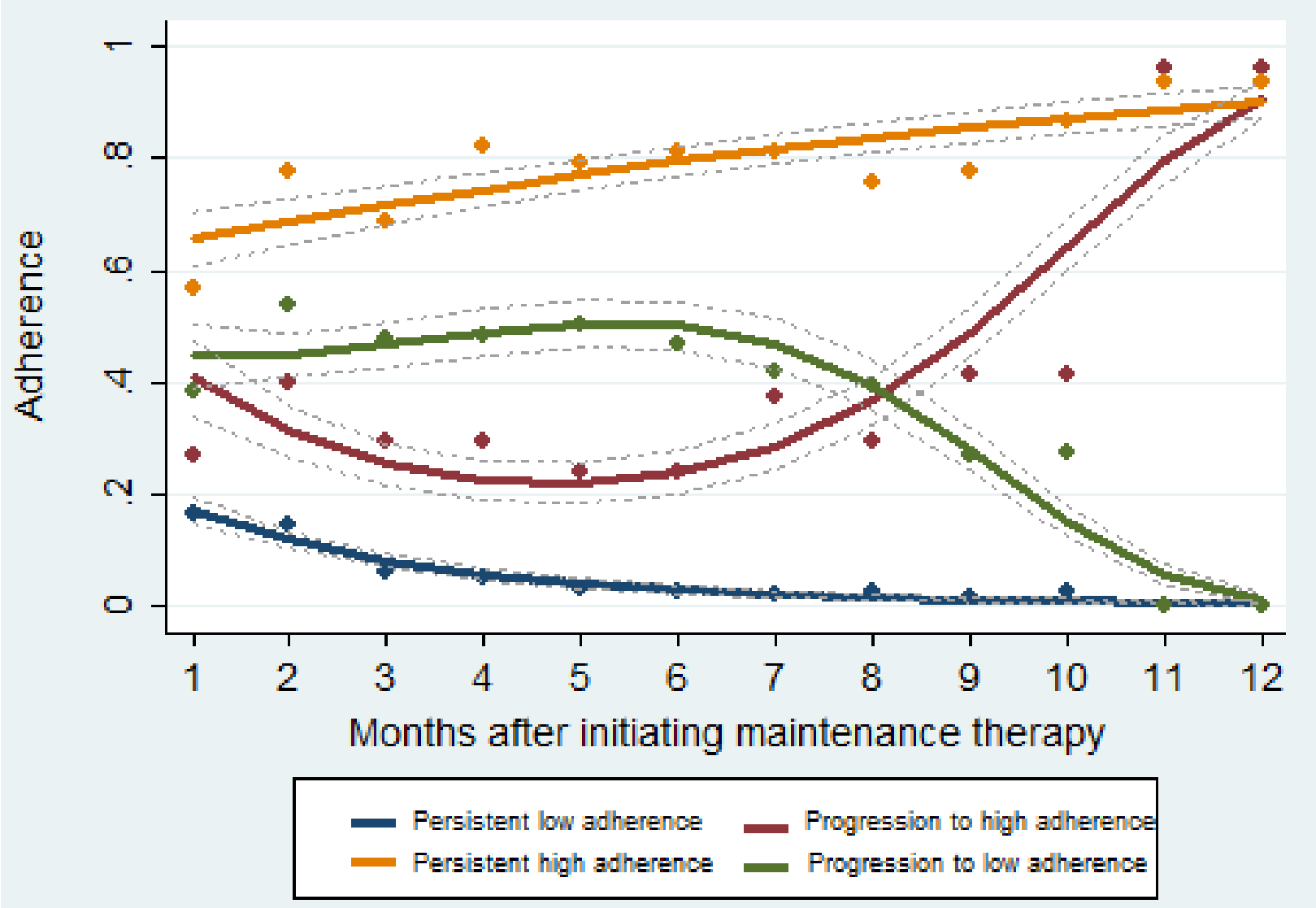




\section{CHAPTER 5}

\section{Summary and Conclusion}

\subsection{Summary of Findings and Discussion}

\section{Prevalence of ACO}

In our study, the overall prevalence of ACO was $1.5 \%$ among community dwelling Medicare beneficiaries. Although no study has reported the specific prevalence of ACO among community dwelling Medicare beneficiaries, this estimate is less than $3.8 \%$ which was previously reported by Kumbhare et al for (65-85 years old) older adults in the United States14. Possible explanations of this difference are methods of identification and time period. While this study used fee-for-service Medicare claims for identification of ACO during a calendar year Kumbhare et. Al. used "ever diagnosed" using self-reports. Thus, we may have captured only older adults who had received healthcare services for asthma and COPD.

Excess Economic Burden of ACO in Terms of Total Health Care Expenditures and Third-

\section{Party Payers' Expenditures}

In terms of projected total healthcare expenditures, nearly two billion dollars (2013USD) were spent on healthcare (weighted number of ACO adults in $2013=42,984$ multiplied by the average per-capita cost $\$ 45,532$ ) of older community-dwelling adults with ACO. In terms of the excess economic burden (i.e. total health care expenditures and third-party payers' expenditures) individuals with ACO had almost four, two, and 1.5 times higher expenditures compared to individuals with NANC, asthma only, and COPD only. Even after controlling for multiple determinants of expenditures, the presence of ACO was associated with significantly higher expenditures in comparison with individuals with NANC, asthma only, and COPD only - 
consistent with published studies that compared per-capita expenditures of individuals with ACO to those with asthma only17 or those with COPD only 18.

The excess expenditures associated with ACO is not surprising because studies have documented increasing expenditures with an increasing number of chronic conditions 80. Furthermore, our finding that ACO patients had higher economic burden compared those with asthma only or COPD only suggest that ACO adults may have higher rates healthcare utilization including hospitalization and emergency department/urgent care visits, which contribute to the higher expenditures. In a cohort of 17,088 COPD patients in Taiwan, ACO patients experienced almost twice as many acute respiratory events (e.g. acute respiratory failure, pneumonia, and acute exacerbation) than did the rest of the COPD cohort12.

\section{Excess Economic Burden of ACO for Patients and Their Families: Out-of-Pocket Healthcare Expenditures}

In this study, those with ACO had higher odds of high OOP spending burden compared to all other groups in both unadjusted and adjusted models. This high burden could be due to cost-sharing of Medicare-covered services, including prescription drugs and payments for noncovered services by Medicare. OOP burden may be particularly high among individuals with ACO because of high cost-sharing of inhaler medications. Based on data from nationwide Part D plans, it has been estimated that mean OOP expenditures were \$30-\$105 per inhaler in 201564 . This means that a typical older Medicare beneficiary with ACO who is prescribed 2-3 inhalers per month has annual OOP expenditures of at least $\$ 1,622-\$ 2,811$ for his/her inhalers64.

Individuals with ACO may have greater need for some of the services not covered by Medicare (example: dental, hearing, and vision) compared to NANC as they are higher risk of these conditions. For example, individuals with $\mathrm{ACO}$ have significantly higher odds (AOR = 
$1.82,95 \% \mathrm{CI}=[1.59,2.08], \mathrm{P}<0.001)$ of removing all teeth compared to $\mathrm{NANC}_{81}$, suggesting greater spending on oral healthcare by patients. It has also been reported that COPD patients are vulnerable to low-value cares2, which may increase OOP spending of families and patients through cost-sharing. Medicare reform proposals that focus on value-based healthcare that balance the value of health services to the OOP spending burden of patients may reduce the excess economic burden of patients.

\section{Contribution of Differences in Patient Characteristics to the Excess Economic Burden of} ACO

The current study used linear and non-linear decomposition analyses to examine the drivers of the excess economic burden of chronic respiratory conditions, one of the top four conditions of public health concern designated by the World Health Organization (WHO)83. The other three conditions are cardiovascular disease, diabetes, and cancer. It has to be noted that older adults with chronic respiratory conditions also have higher rates of CVD, diabetes, and cancer. A population survey among ( $\geq 18$ years) adults in the US also found that the adjusted odds of six chronic conditions (e.g. cardiovascular diseases, diabetes, and depression) is higher in ACO compared to asthma only and COPD only 14. In our study, $91 \%$ of ACO patients had more than two other chronic conditions which was higher than asthma only (89\%), COPD only (87\%) and NANC (76\%) groups. Our decomposition analysis suggested that patient-level characteristics such as higher number of co-existing chronic conditions and related characteristics (i.e., higher number of unique medications, and higher levels of fragmented care) were the leading drivers of the excess economic burden among ACO patients. For example, receiving care from several different providers often results in gaps in communication between providers which can lead to suboptimal care and polypharmacy75,84. Prior studies suggest that 
more fragmented care is associated with more diagnostic tests, more low-value care, and lower patient satisfaction85-87. Previous studies further suggest the associations of fragmented care and higher number of emergency department (ED) visits, higher hospitalizations, and higher expenditures 77,88 .

\section{Cost-Related Medication Non-Adherence and ACO}

One in six older Medicare beneficiaries with ACO reported any CRN between 2006 and 2013. The most prevalent form of CRN was "failing to fill prescription," which was almost twice as high among Medicare beneficiaries with ACO compare to those with no-asthma and noCOPD. Older adults with ACO were more likely to report any CRN compared to older adults with no-asthma no-COPD; while not directly studied, this likely has significant later-in-life morbidity and mortality consequences76,98. This is not surprising because older adults with ACO compared to those with neither asthma nor COPD had higher OOP burden, and high OOP burden was significantly associated with CRN. The high OOP burden among adults with ACO can be explained by the high cost of brand-name inhaler therapies and the high medication burden per individualized regimen to adequately manage $\mathrm{ACO}$ (often two to three inhaler therapies).

\section{Role of Number of Medication in High Risk of CRN among Patients with ACO}

In our study, increased risk of any CRN among ACO individuals compared to those with no-asthma no-COPD is apparent by adjusting all factors without number of unique medications. However, the inclusion of number of unique medications attenuated this association, suggesting that number of medications may be a key link between ACO and CRN. Notably, the mean number of unique medications is approximately 16 among individuals with ACO which is significantly higher compared to individuals with asthma-only ( 13), COPD-only ( 12.5) and no-asthma no-COPD ( 7). Individuals with ACO have a higher number of unique medications 
due to two following reasons: (1) individuals with ACO may need to use a higher number of respiratory medications to adequately control respiratory symptoms. The Latin American Project for the Investigation of Obstructive Lung Disease (PLATINO) study found that $49 \%$ of individuals with ACO used respiratory medications significantly higher than individuals with asthma-only (38\%) or COPD-only (20\%)13; (2) ACO individuals may need to use a greater number of non-respiratory medications due to higher risk of comorbid medical conditions. In our study, $43 \%$ of ACO individuals had 4-6 other chronic conditions, while this number was lower among individuals with asthma-only (33\%), COPD-only (36\%), and no-asthma no-COPD (23\%). A population survey in the US similarly found that $90 \%$ of individuals with ACO had at least one other co-existing condition (compared with $71 \%$ in asthma-only and $84 \%$ in COPDonly) 14 .

\section{Trajectories of Adherence to IMT among Patients with ACO}

We identified four distinct patterns of medication adherence: (1) persistent low adherence; (2) persistent high adherence; (3) progression to low adherence; (4) and progression to high adherence. The majority of older adults (57\%) had persistent poor adherence and only $11 \%$ had "persistent high adherence." As there are no published studies on medication adherence trajectories over time among older adults with ACO, we compare our results to studies that focused on adults with asthma or COPD. Our study findings are consistent with published studies that have reported low adherence to maintenance therapy among individuals with either asthma or COPD. A recent study conducted in Australia reported that the "persistent low adherence" trajectory to IMT was observed among 58\% of individuals with asthma who were between 12 and 45 years old 25. Studies that use PDC for measuring medication adherence also reported the high prevalence of medication non-adherence ( $<80 \%$ PDC) among patients with 
asthma or COPD. For example, a systematic review of 39 clinical trials reported that only $47 \%$ to $57 \%$ of individuals with asthma were adherent to maintenance therapy27. Similarly, a study of 244 patients with COPD reported that adherence to maintenance therapy with ICS/LABA was less than $23 \% 28$. Poor adherence to maintenance therapy was reported particularly higher among the elderly population. In a retrospective cohort study on Medicare beneficiaries with COPD, the average monthly adherence to IMT was plateaued at 0.35 by the seventh month 31 .

\section{Type of IMT and Trajectories of Adherence among Patients with ACO}

In our study cohort of older adults with ACO, $26.8 \%$ were initiated on ICS monotherapy and $73.2 \%$ were initiated on ICS/LABA. This means that the percentage of older adults with ACO who used ICS/LABA for IMT is three times higher than those who used ICS for IMT. An important finding of this study is that older adults who were initiated on ICS/LABA were less likely to have "persistent low adherence". Previous studies among patients with asthma also support this finding. A clinical trial conducted by Perrin et al. reported that adherence to ICS/LABA was higher as compared to ICS monotherapy142. Similarly, two other studies among the patient with asthma concluded that that use of ICS/LABA is likely to improve adherence to IMT compared with ICS 120,121. As stated in the Introduction, ICS/LABA confers many advantages. Although many patients perceive that inhalers are effective in relieving the symptom, this perceived efficacy of ICS/LABA may be higher. This higher perceived efficacy can be due to a faster improvement of symptoms by using ICS/LABA. A previous clinical trial among patients with asthma has reported that ICS/LABA resulted in a faster improvement in asthma symptoms compared to ICS monotherapy for IMT143.

\subsection{Implications and Suggestions for Future Research}


Our study findings highlight the need for a "holistic management" of older adults with chronic respiratory conditions, specifically ACO, asthma, and COPD. Given many with chronic respiratory conditions need to see multiple providers and use multiple medications concurrently (example: inhaled corticosteroids, short- and long acting beta agonist) for managing their respiratory symptoms 110 and other medications to manage their chronic conditions), the risks of fragmented care and drug-disease and drug-drug interactions are very high. These factors often lead greater economic burden, as evidenced by our study. Although CMS began reimbursing providers for providing coordinated care for those with multiple conditions in 201589 , it remains to be seen whether such fiscal incentives for care coordination have resulted in less fragmented care and reduce healthcare expenditures. In this context, patients with chronic respiratory conditions (ACO, and COPD) may need to be managed in patient-centered medical homes that are "comprehensive, team-based, coordinated, accessible, and focused on quality and safety." 90. As documented in published literature, pharmacists can play an important role in medical homes by counseling patients and/or caregivers, reviewing inhaler techniques, simplifying medication regiments, and providing adherence assessments91-93. Such management may improve not only outcomes, but also lower the economic burden of patients, payers, and health systems.

Our study's findings highlight the need for developing guidelines for management of multiple chronic conditions. Applying "single-disease focused" clinical practice guidelines can result in adverse drug-drug and drug-disease interactions. A systematic review of recommendations in $12 \mathrm{UK}$ national clinical guidelines identified that potentially serious drugdrug interactions were common94. Adherence to guidelines for those with multiple chronic conditions can also generate more low-value care and lead to higher healthcare expenditures the 
patient, payers, and the society95,96. Guidelines which focus on overlapping or larger scale care would benefit patients with multiple comorbidities.

Reducing the number of medications may directly reduce the likelihood of CRN and indirectly alleviate the high burden of OOP prescription cost among older adults with ACO. As documented in published literature, clinicians and pharmacists can play an important role in reducing the number of medications. For example, during annual wellness visits—now covered by Medicare - a comprehensive review of medications can be performed and highlights opportunities for prescription regimen optimization113. Providers can proactively participate in deprescribing by: (1) discontinuing unnecessary therapy, (2) discontinuing duplicate therapies, (3) combining therapies as appropriate (i.e. combination inhalers), and (4) replacing high-cost therapies with more affordable options. In a survey of elderly Medicare beneficiaries, more than $30 \%$ of individuals reported they did not have the chance of talking with their provider about their medications in the last 12 months114. Intensive, chronic care management can be optimized through team-based care.

The results of this study suggest that the adherence to IMT varies over time. For example, one third of patients changed their adherence behavior after 6 months of starting IMT. Hence, there is a need to use appropriate adherence interventions at the right time. Different interventions can be used to address poor adherence among patients with ACO. Interventions such as motivational interviewing 138 , coaching and patient education 139, shared decisionmaking 140 , and simplification of regimen 141 can be used for improving adherence among patients with ACO as these interventions have successfully improved adherence among patients with asthma or COPD. For designing personalized medication adherence intervention at the right time, it is important to focus on underlying factors associated with poor adherence. Previous 
studies have suggested that patient-related, socioeconomic-related, health system-related, condition-related, and therapy-related are contributed to poor adherence33. Among all these factors, therapy related factors may be the least expensive modifiable factors among all.

Frequency, length, and complexity of regimen are some of the many therapy-related factors that can impact adherence among patients with chronic pulmonary diseases 144 . One important therapy-related factor for IMT among individuals with ACO may be IMT type. Future randomized clinical trials are needed to investigate the differences in improvement of symptoms between ICS/LABA and ICS for IMT of ACO.

\subsection{Strengths and Limitations}

The strengths of this study are: (1) use of a nationally representative survey of elderly Medicare beneficiaries linked to their fee-for-service claims data (Aims $1 \& 2$ ); (2) inclusion of a comprehensive set of factors by using a strong framework for all aims; (3) examination of leading drivers of the excess burden with advanced econometric techniques; (4) contribution to knowledge gap by being the first study to examine the impact of medication type on adherence to IMT (Aim 3); (5) use of real-world data from Medicare advantage database adults (Aim 3); (6) adoption of longitudinal study design with adjustments for observed selection bias by using IPTW (Aim 3) and; (7) and use of GBTM techniques (Aim 3) to identify adherence trajectories over time.

Some limitations of the proposed research should be noted. Lack of information on the severity of asthma and COPD is a limitation of this study. While some of the advanced methods shed light on drivers of the excess economic burden and medication adherence over time, they cannot explain reasons behind these issues. The study findings are not generalizable to all older adults. Furthermore Aims 1 and 2 used cross sectional design and therefore cannot be used for 
establishing causal inference. Our measure of CRN was not specific to ACO medications. In aim 3, we did not have information on sociodemographic characteristics which may affect adherence and therefore we may have overestimated they effect of IMT type on adherence over time. Furthermore, by using prescription fill data (Aim 3) and not actual use of medications to define adherence, we may have not accurately measured medication adherence.

\subsection{Conclusion}

Overall among older adults, ACO was associated with excess economic burden (in terms of total healthcare, third-party expenditures and OOP spending burden), even after adjusting with a comprehensive list of variables. Post regression decomposition analyses revealed multiple medications use and high levels of fragmented care were the leading drivers of the excess economic burden. Older adults with ACO were also vulnerable for any CRN and its specific forms. The high number of medications used among ACO individuals explained the association of ACO to CRN. Furthermore, only one in 10 older adults with ACO had "persistent high adherence" to IMT and nearly six in 10 older adults had "persistent low adherence" to IMT. Our study's findings suggest that using ICS/LABA for IMT may reduce the risk of "persistent low adherence" over time among older adults with ACO. Individual, policy, and program interventions that facilitate coordination of care may reduce the excess economic burden, and CRN of older adults with chronic respiratory conditions, specifically ACO. Our study's findings highlight the need for heightened surveillance of ACO patients, policies and programs to reduce the economic burden on patients, and holistic management of ACO patients within integrated systems of healthcare delivery. Future prospective cohort studies are needed to confirm the study findings as well as to identify reasons for non-adherence to inform intervention efforts. 


\section{References}

1. Ferkol T, Schraufnagel D. The global burden of respiratory disease. Ann Am Thorac Soc. 2014;11(3):404-406. doi:10.1513/AnnalsATS.201311-405PS

2. Soriano JB, Abajobir AA, Abate KH, et al. Global, regional, and national deaths, prevalence, disability-adjusted life years, and years lived with disability for chronic obstructive pulmonary disease and asthma, 1990-2015: a systematic analysis for the Global Burden of Disease Study 2015. Lancet Respir Med. 2017;5(9):691-706. doi:10.1016/S2213-2600(17)30293-X

3. Nurmagambetov T, Kuwahara R, Garbe P. The economic burden of asthma in the United States, 2008-2013. Ann Am Thorac Soc. 2018;15(3):348-356.

doi:10.1513/AnnalsATS.201703-2590C

4. Guarascio AJ, Ray SM, Finch CK, Self TH. The clinical and economic burden of chronic obstructive pulmonary disease in the USA. Clin Outcomes Res. 2013;5(1):235-245. doi:10.2147/CEOR.S34321

5. Gibson PG, Simpson JL. The overlap syndrome of asthma and COPD: What are its features and how important is it? Thorax. 2009;64(8):728-735.

doi:10.1136/thx.2008.108027

6. de Marco R, Pesce G, Marcon A, et al. The Coexistence of Asthma and Chronic Obstructive Pulmonary Disease (COPD): Prevalence and Risk Factors in Young, Middleaged and Elderly People from the General Population. PLoS One. 2013;8(5). doi:10.1371/journal.pone.0062985

7. Global Initiative for Asthma GI for COLD (GOLD). Asthma, COPD, and asthma-COPD overlap. https://ginasthma.org/wp-content/uploads/2019/11/GINA-GOLD-2017-overlappocket-guide-wms-2017-ACO.pdf. Published 2017. Accessed October 4, 2020.

8. Enrique DG, Khosravi M, Mannino DM. Asthma, chronic obstructive pulmonary disease, and mortality in the U.S. population. COPD J Chronic Obstr Pulm Dis. 2011;8(6):400407. doi:10.3109/15412555.2011.611200

9. Hosseini M, Almasi-Hashiani A, Sepidarkish M, Maroufizadeh S. Global prevalence of asthma-COPD overlap (ACO) in the general population: A systematic review and metaanalysis. Respir Res. 2019;20(1):229. doi:10.1186/s12931-019-1198-4

10. Soriano JB, Davis KJ, Coleman B, Visick G, Mannino D, Pride NB. The proportional venn diagram of obstructive lung disease: Two approximations from the United States and the United Kingdom. Chest. 2003;124(2):474-481. doi:10.1378/chest.124.2.474

11. Fu JJ, Gibson PG, Simpson JL, McDonald VM. Longitudinal changes in clinical outcomes 
in older patients with asthma, COPD and asthma-COPD overlap syndrome. Respiration. 2014;87(1):63-74. doi:10.1159/000352053

12. Chung WS, Lin CL, Kao CH. Comparison of acute respiratory events between: AsthmaCOPD overlap syndrome and COPD patients. Med (United States). 2015;94(17):e755. doi:10.1097/MD.0000000000000755

13. Menezes AMB, De Oca MM, Pérez-Padilla R, et al. Increased risk of exacerbation and hospitalization in subjects with an overlap phenotype : COPD-Asthma. Chest. 2014;145(2):297-304. doi:10.1378/chest.13-0622

14. Kumbhare S, Pleasants R, Ohar JA, Strange C. Characteristics and prevalence of asthma/chronic obstructive pulmonary disease overlap in the United States. Ann Am Thorac Soc. 2016;13(6):803-810. doi:10.1513/AnnalsATS.201508-554OC

15. Rhee CK, Yoon HK, Yoo KH, et al. Medical utilization and cost in patients with overlap syndrome of chronic obstructive pulmonary disease and asthma. COPD J Chronic Obstr Pulm Dis. 2014;11(2):163-170. doi:10.3109/15412555.2013.831061

16. Shantakumar S, Pwu R-F, D'Silva L, et al. Burden of asthma and COPD overlap (ACO) in Taiwan: a nationwide population-based study. BMC Pulm Med. 2018;18(1):16. doi:10.1186/s12890-017-0571-7

17. Gerhardsson De Verdier M, Andersson M, Kern DM, Zhou S, Tunceli O. Asthma and Chronic Obstructive Pulmonary Disease Overlap Syndrome: Doubled Costs Compared with Patients with Asthma Alone. Value Heal. 2015;18(6):759-766.

doi:10.1016/j.jval.2015.04.010

18. Blanchette CM, Gutierrez B, Ory C, Chang E, Akazawa M. Economic burden in direct costs of concomitant chronic obstructive pulmonary disease and asthma in a Medicare Advantage population. J Manag Care Pharm. 2008;14(2):176-185.

doi:10.18553/jmcp.2008.14.2.176

19. Ishiura Y, Fujimura M, Shiba Y, Ohkura N, Hara J, Kasahara K. A comparison of the efficacy of once-daily fluticasone furoate/vilanterole with twice-daily fluticasone propionate/salmeterol in asthma-COPD overlap syndrome. Pulm Pharmacol Ther. 2015;35:28-33. doi:10.1016/J.PUPT.2015.10.005

20. Jardim JR, Nascimento OA. The Importance of Inhaler Adherence to Prevent COPD Exacerbations. Med Sci. 2019;7(4):54. doi:10.3390/medsci7040054

21. Engelkes M, Janssens HM, de Jongste JC, Sturkenboom MCJM, Verhamme KMC. Medication adherence and the risk of severe asthma exacerbations: a systematic review. Eur Respir J. 2015;45(2):396 LP - 407. doi:10.1183/09031936.00075614 
22. Chisholm-Burns MA, Kim Lee J, Spivey CA, et al. US Pharmacists' Effect as Team Members on Patient Care. Med Care. 2010;48(10):923-933.

doi:10.1097/MLR.0b013e3181e57962

23. Osterberg L, Blaschke T. Adherence to medication. N Engl J Med. 2005;353(5):487-497. doi:10.1056/NEJMra050100

24. Simoni-Wastila L, Wei YJ, Qian J, et al. Association of chronic obstructive pulmonary disease maintenance medication adherence with all-cause hospitalization and spending in a medicare population. Am J Geriatr Pharmacother. 2012;10(3):201-210.

doi:10.1016/j.amjopharm.2012.04.002

25. van Boven JFM, Koponen M, Lalic S, et al. Trajectory Analyses of Adherence Patterns in a Real-Life Moderate to Severe Asthma Population. J Allergy Clin Immunol Pract. 2020;0(0). doi:10.1016/j.jaip.2019.12.002

26. DiMatteo MR. Variations in Patients' Adherence to Medical Recommendations: A Quantitative Review of 50 Years of Research. Med Care. 42:200-209. doi:10.2307/4640729

27. Normansell R, Kew KM, Stovold E. Interventions to improve adherence to inhaled steroids for asthma. Cochrane Database Syst Rev. 2017;2017(4).

doi:10.1002/14651858.CD012226.pub2

28. Sulaiman I, Cushen B, Greene G, et al. Objective assessment of adherence to inhalers by patients with chronic obstructive pulmonary disease. Am J Respir Crit Care Med. 2017;195(10):1333-1343. doi:10.1164/rccm.201604-0733OC

29. Mueller S, Wilke T, Bechtel B, Punekar YS, Mitzner K, Virchow JC. Non-persistence and non-adherence to long-acting COPD medication therapy: A retrospective cohort study based on a large German claims dataset. Respir Med. 2017;122:1-11. doi:10.1016/j.rmed.2016.11.008

30. Jung E, Pickard AS, Salmon JW, Bartle B, Lee TA. Medication adherence and persistence in the last year of life in COPD patients. Respir Med. 2009;103(4):525-534.

doi:10.1016/j.rmed.2008.11.004

31. Albrecht JS, Park Y, Hur P, et al. Adherence to maintenance medications among older adults with chronic obstructive pulmonary disease the role of depression. Ann Am Thorac Soc. 2016;13(9):1497-1504. doi:10.1513/AnnalsATS.201602-136OC

32. Castaldi PJ, Rogers WH, Safran DG, Wilson IB. Inhaler costs and medication nonadherence among seniors with chronic pulmonary disease. Chest. 2010;138(3):614620. doi:10.1378/chest.09-3031 
33. Organization WH, ed. Adherence to Long-Term Therapies: Evidence for Action. Geneva: World Health Organization; 2003.

34. Kyle MA, Blendon RJ, Benson JM, Abrams MK, Schneider EC. Financial Hardships Of Medicare Beneficiaries With Serious Illness. Health Aff (Millwood). 2019;38(11):18011806. doi:10.1377/hlthaff.2019.00362

35. George M. Adherence in asthma and copd: New strategies for an old problem. Respir Care. 2018;63(6):818-831. doi:10.4187/respcare.05905

36. Moon M. What Medicare has meant to older Americans. Health Care Financ Rev. 1996;18(2):49-59.

37. Luiza VL, Chaves LA, Silva RM, et al. Pharmaceutical policies: Effects of cap and copayment on rational use of medicines. Cochrane Database Syst Rev. 2015;2015(5). doi:10.1002/14651858.CD007017.pub2

38. Goedken AM, Urmie JM, Farris KB, Doucette WR. Impact of cost sharing on prescription drugs used by Medicare beneficiaries. Res Soc Adm Pharm. 2010;6(2):100-109.

doi:10.1016/j.sapharm.2010.03.003

39. Cotton P, Newhouse JP, Volpp KG, et al. Medicare Advantage: Issues, Insights, and Implications for the Future. Popul Health Manag. 2016;19(S3):S-1-S-8. doi:10.1089/pop.2016.29013.pc

40. Levine DA, Morgenstern LB, Langa KM, Piette JD, Rogers MAM, Karve SJ. Recent trends in cost-related medication nonadherence among stroke survivors in the United States. Ann Neurol. 2013;73(2):180-188. doi:10.1002/ana.23823

41. Harrold LR, Briesacher BA, Peterson D, et al. Cost-related medication nonadherence in older patients with rheumatoid arthritis. J Rheumatol. 2013;40(2):137-143. doi:10.3899/jrheum.120441

42. Zhang JX. Risk factors for cost-related medication non-adherence among older patients with diabetes. World J Diabetes. 2014;5(6):945. doi:10.4239/wjd.v5.i6.945

43. Rau JL. Determinants of patient adherence to an aerosol regimen. Respir Care. 2005;50(10):1346-1356; discussion 1357-9.

44. Restrepo RD, Alvarez MT, Wittnebel LD, et al. Medication adherence issues in patients treated for COPD. Int J COPD. 2008;3(3):371-384. doi:10.2147/copd.s3036

45. George M, Topaz M, Rand C, et al. Inhaled corticosteroid beliefs, complementary and alternative medicine, and uncontrolled asthma in urban minority adults. J Allergy Clin Immunol. 2014;134(6):1252-1259. doi:10.1016/j.jaci.2014.07.044 
46. Gershon AS, Campitelli MA, Croxford R, et al. Combination long-acting $\beta$-agonists and inhaled corticosteroids compared with long-acting $\beta$-agonists alone in older adults with chronic obstructive pulmonary disease. JAMA - J Am Med Assoc. 2014;312(11):11141121. doi:10.1001/jama.2014.11432

47. Amegadzie JE, Gorgui J, Acheampong L, Gamble JM, Farrell J, Gao Z. Comparative safety and effectiveness of inhaled bronchodilators and corticosteroids for treating asthma-COPD overlap: a systematic review and meta-analysis. J Asthma. 2019. doi:10.1080/02770903.2019.1687716

48. $\mathrm{Wu} \mathrm{AC}$, Butler MG, Li L, et al. Primary adherence to controller medications for asthma is poor. Ann Am Thorac Soc. 2015;12(2):161-166. doi:10.1513/AnnalsATS.201410-459OC

49. Eppig FJ, Chulis GS. Matching MCBS (Medicare Current Beneficiary Survey) and Medicare data: the best of both worlds. Health Care Financ Rev. 1997;18(3):211-229. doi:hcfr-18-3-211 [pii]

50. Andersen RM. Revisiting the behavioral model and access to medical care: does it matter? J Health Soc Behav. 1995;36(1):1-10. doi:10.2307/2137284

51. Adler GS. A profile of the Medicare Current Beneficiary Survey. Health Care Financ Rev. 1994;15(4):153-163.

52. Optum. Clinformatics Data Mart. https://www.optum.com/content/dam/optum/resources/productSheets/Clinformatics_for_ Data_Mart.pdf. Published 2020. Accessed October 4, 2020.

53. Fragoso CAV, Murphy TE, Agogo GO, Allore HG, McAvay GJ. Asthma-COPD overlap syndrome in the US: a prospective population-based analysis of patient-reported outcomes and health care utilization. Int J Chron Obstruct Pulmon Dis. 2017;12:517.

54. Carroll A. The "iron triangle" of health care: access, cost, and quality. In: JAMA Health Forum. Vol 1. American Medical Association; 2012.

55. Bates DW, Saria S, Ohno-Machado L, Shah A, Escobar G. Big data in health care: using analytics to identify and manage high-risk and high-cost patients. Health Aff. 2014;33(7):1123-1131.

56. Stone PW, Glied SA, McNair PD, et al. CMS changes in reimbursement for HAIs: setting a research agenda. Med Care. 2010;48(5):433.

57. Shih T, Chen LM, Nallamothu BK. Will Bundled Payments Change Health Care? Examining the Evidence Thus Far in Cardiovascular Care. Circulation. 2015;131(24):2151-2158. doi:10.1161/CIRCULATIONAHA.114.010393

58. Frick KD, Kymes SM. The calculation and use of economic burden data. $\mathrm{Br} J$ 
Ophthalmol. 2006;90(3):255-257. doi:10.1136/bjo.2005.084848

59. Rabarison KM, Bish CL, Massoudi MS, Giles WH. Economic Evaluation Enhances Public Health Decision Making. Front public Heal. 2015;3:164.

doi:10.3389/fpubh.2015.00164

60. Rezayatmand R, Pavlova M, Groot W. The impact of out-of-pocket payments on prevention and health-related lifestyle: a systematic literature review. Eur J Public Health. 2012;23(1):74-79. doi:10.1093/eurpub/cks034

61. Baird KE. Recent trends in the probability of high out-of-pocket medical expenses in the United States. SAGE Open Med. 2016;4:2050312116660329. doi:10.1177/2050312116660329

62. Singletary M. Seniors report spending $\$ 22$ billion from savings to cover health-care costs The Washington Post. The Washington Post. https://www.washingtonpost.com/business/2019/04/22/seniors-report-spending-billionsavings-cover-health-care-costs/. Published 2019. Accessed July 5, 2020.

63. Jacobson G, Griffin S, Neuman T, Smith K. Income and assets of Medicare beneficiaries, 2016-2035. Menlo Park CA Kaiser Fam Found goo gl/HjXVSQ Retrieved January. 2017;26:2018.

64. Tseng C-W, Yazdany J, Dudley RA, et al. Medicare Part D plans' coverage and costsharing for acute rescue and preventive inhalers for chronic obstructive pulmonary disease. JAMA Intern Med. 2017;177(4):585-588.

65. Reschovsky JD, Hadley J, Saiontz-Martinez CB, Boukus ER. Following the money: factors associated with the cost of treating high-cost Medicare beneficiaries. Health Serv Res. 2011;46(4):997-1021. doi:10.1111/j.1475-6773.2011.01242.x

66. Dieleman JL, Squires E, Bui AL, et al. Factors Associated With Increases in US Health Care Spending, 1996-2013. JAMA. 2017;318(17):1668-1678. doi:10.1001/jama.2017.15927

67. Ajmera M, Raval AD, Shen C, Sambamoorthi U. Explaining the increased health care expenditures associated with gastroesophageal reflux disease among elderly Medicare beneficiaries with chronic obstructive pulmonary disease: a cost-decomposition analysis. Int J Chron Obstruct Pulmon Dis. 2014;9:339-348. doi:10.2147/COPD.S59139

68. Tamblyn R. A Novel Personalized Fall Risk Calculator: A Prototype for Improving the Safety of Prescribing through Computerized Decision Support.; 2016. doi:10.1007/978-3319-32304-6_20

69. Jones SMW, Chennupati S, Nguyen T, Fedorenko C, Ramsey SD. Comorbidity is 
associated with higher risk of financial burden in Medicare beneficiaries with cancer but not heart disease or diabetes. Medicine (Baltimore). 2019;98(1):e14004-e14004. doi:10.1097/MD.0000000000014004

70. Schoen C, Davis K, Willink A. Medicare Beneficiaries' High Out-of-Pocket Costs: Cost Burdens by Income and Health Status. Issue Brief (Commonw Fund). 2017;11:1-14.

71. US Bureau of labor statistics. Measuring Price Change in the CPI: Medical care. Division of Consumer Prices and Price Indexes. https://www.bls.gov/cpi/factsheets/medicalcare.htm. Published 2020.

72. Crystal S, Johnson R, Harman J, Sambamoorthi U, Kumar. R. Out-of-pocket health care costs among older Americans. Journals Gerontol Ser B. 2000;55(1):S51-S62. doi:10.1093/geronb/55.1.S51

73. Van Dyke MK, Hinds D, Dickinson H, Sansbury L. Evaluation of ICD-9 to ICD-10 Conversion on Estimates of Asthma and COPD in a US Commercial Claims Database. In: C37. OPTIMIZING ASTHMA CARE ACROSS DIVERSE PATIENTS. ; 2018:A4868A4868. doi:doi:10.1164/ajrccm-conference.2018.197.1_MeetingAbstracts.A4868

74. Wurst KE, St. Laurent S, Hinds D, Davis KJ. Disease Burden of Patients with Asthma/COPD Overlap in a US Claims Database: Impact of ICD-9 Coding-based Definitions. COPD J Chronic Obstr Pulm Dis. 2017;14(2):200-209. doi:10.1080/15412555.2016.1257598

75. Kern LM, Seirup JK, Rajan M, Jawahar R, Stuard SS. Fragmented ambulatory care and subsequent healthcare utilization among Medicare beneficiaries. Am J Manag Care. 2018;24(9):e278-e284.

76. Gupta S, McColl MA, Guilcher SJ, Smith K. Cost-related nonadherence to prescription medications in Canada: A scoping review. Patient Prefer Adherence. 2018;12:1699-1715. doi:10.2147/PPA.S170417

77. Liu CW, Einstadter D, Cebul RD. Care fragmentation and emergency department use among complex patients with diabetes. Am J Manag Care. 2010;16(6):413-420.

78. Liu S, Yeung PC. Measuring fragmentation of ambulatory care in a tripartite healthcare system. BMC Health Serv Res. 2013;13:176. doi:10.1186/1472-6963-13-176

79. Fairlie RW. An extension of the Blinder-Oaxaca decomposition technique to logit and probit models. J Econ Soc Meas. 2005;30(4):305-316.

80. Krishnan JA, Nibber A, Chisholm A, et al. Prevalence and Characteristics of AsthmaChronic Obstructive Pulmonary Disease Overlap in Routine Primary Care Practices. Ann Am Thorac Soc. 2019;16(9):1143-1150. 
81. Dwibedi N, Wiener RC, Findley PA, Shen C, Sambamoorthi U. Asthma, chronic obstructive pulmonary disease, tooth loss, and edentulism among adults in the United States: 2016 Behavioral Risk Factor Surveillance System survey. J Am Dent Assoc. 2019;0(0). doi:10.1016/j.adaj.2019.07.032

82. Spece LJ, Donovan LM, Griffith MF, et al. Initiating Low-Value Inhaled Corticosteroids in an Inception Cohort with Chronic Obstructive Pulmonary Disease. Ann Am Thorac Soc. 2020;17(5):589-595. doi:10.1513/AnnalsATS.201911-854OC

83. Organization WH. Tackling NCDs: 'best Buys' and Other Recommended Interventions for the Prevention and Control of Noncommunicable Diseases. World Health Organization; 2017.

84. O'Malley AS, Reschovsky JD. Referral and consultation communication between primary care and specialist physicians: finding common ground. Arch Intern Med. 2011;171(1):5665.

85. Kern LM, Seirup JK, Casalino LP, Safford MM. Healthcare fragmentation and the frequency of radiology and other diagnostic tests: a cross-sectional study. J Gen Intern Med. 2017;32(2):175-181.

86. Romano MJ, Segal JB, Pollack CE. The association between continuity of care and the overuse of medical procedures. JAMA Intern Med. 2015;175(7):1148-1154.

87. Van Walraven C, Oake N, Jennings A, Forster AJ. The association between continuity of care and outcomes: a systematic and critical review. J Eval Clin Pract. 2010;16(5):947956.

88. Hussey PS, Schneider EC, Rudin RS, Fox DS, Lai J, Pollack CE. Continuity and the costs of care for chronic disease. JAMA Intern Med. 2014;174(5):742-748.

89. Department of Health and Human Services. Connected care toolkit: chronic care management resources for health care professionals and communities.

90. Ortiz G, Fromer L. Patient-Centered Medical Home in chronic obstructive pulmonary disease. J Multidiscip Healthc. 2011;4:357-365. doi:10.2147/JMDH.S22811

91. Elliott RA, O’Callaghan C, Paul E, George J. Impact of an intervention to reduce medication regimen complexity for older hospital inpatients. Int J Clin Pharm. 2013;35(2):217-224. doi:10.1007/s11096-012-9730-3

92. Muir AJ, Sanders LL, Wilkinson WE, Schmader K. Reducing medication regimen complexity: a controlled trial. J Gen Intern Med. 2001;16(2):77-82. doi:10.1046/j.15251497.2001.016002077.x

93. Newsome AS, Smith SE, Olney WJ, et al. Medication regimen complexity is associated 
with pharmacist interventions and drug-drug interactions: A use of the novel MRC-ICU scoring tool. JACCP J Am Coll Clin Pharm. 2020;3(1):47-56. doi:10.1002/jac5.1146

94. Dumbreck S, Flynn A, Nairn M, et al. Drug-disease and drug-drug interactions: systematic examination of recommendations in $12 \mathrm{UK}$ national clinical guidelines. BMJ.

2015;350:h949-h949. doi:10.1136/bmj.h949

95. Brownlee S, Chalkidou K, Doust J, et al. Evidence for overuse of medical services around the world. Lancet (London, England). 2017;390(10090):156-168. doi:10.1016/S01406736(16)32585-5

96. Schwartz AL, Landon BE, Elshaug AG, Chernew ME, McWilliams JM. Measuring lowvalue care in Medicare. JAMA Intern Med. 2014;174(7):1067-1076.

doi:10.1001/jamainternmed.2014.1541

97. O’Byrne P, Fabbri LM, Pavord ID, Papi A, Petruzzelli S, Lange P. Asthma progression and mortality: The role of inhaled corticosteroids. Eur Respir J. 2019;54(1).

doi:10.1183/13993003.00491-2019

98. Khera R, Valero-Elizondo J, Das SR, et al. Cost-Related Medication Nonadherence in Adults with Atherosclerotic Cardiovascular Disease in the United States, 2013 to 2017. Circulation. 2019;140(25):2067-2075. doi:10.1161/CIRCULATIONAHA.119.041974

99. Piette JD, Heisler M, Wagner TH. Cost-related medication underuse among chronically III adults: the treatments people forgo, how often, and who is at risk. Am J Public Health. 2004;94(10):1782-1787.

100. Lee S, Jiang L, Dowdy D, Hong YA, Ory MG. Peer Reviewed: Attitudes, Beliefs, and Cost-Related Medication Nonadherence Among Adults Aged 65 or Older With Chronic Diseases. Prev Chronic Dis. 2018;15.

101. Kim E-Y, Han H-R, Jeong S, et al. Does knowledge matter? Intentional medication nonadherence among middle-aged Korean Americans with high blood pressure. $J$ Cardiovasc Nurs. 2007;22(5):397-404. doi:10.1097/01.JCN.0000287038.23186.bd

102. Kang H, Lobo JM, Kim S, Sohn M-W. Cost-related medication non-adherence among US adults with diabetes. Diabetes Res Clin Pract. 2018;143:24-33.

103. Jensen GA, Xu X. Prescription drug insurance and cost-related medication nonadherence among Medicare seniors: findings from two national surveys. J Pharm Heal Serv Res. 2016;7(1):11-21. doi:10.1111/jphs.12116

104. Burcu M, Alexander GC, Ng X, Harrington D. Construct validity and factor structure of survey-based assessment of cost-related medication burden. Med Care. 2015;53(2):199206. 
105. Piette JD, Rosland AM, Silveira MJ, Hayward R, McHorney CA. Medication cost problems among chronically ill adults in the US: Did the financial crisis make a bad situation even worse? Patient Prefer Adherence. 2011;5:187-194.

doi:10.2147/PPA.S17363

106. Heisler M, Choi H, Rosen AB, et al. Hospitalizations and deaths among adults with cardiovascular disease who underuse medications because of cost: A longitudinal analysis. Med Care. 2010;48(2):87-94. doi:10.1097/MLR.0b013e3181c12e53

107. Madden JM, Graves AJ, Zhang F, et al. Cost-related medication nonadherence and spending on basic needs following implementation of Medicare Part D. Jama. 2008;299(16):1922-1928.

108. Soumerai SB, Pierre-Jacques M, Zhang F, et al. Cost-related medication nonadherence among elderly and disabled medicare beneficiaries: a national survey 1 year before the medicare drug benefit. Arch Intern Med. 2006;166(17):1829-1835.

109. Pierre-Jacques M, Safran DG, Zhang F, et al. Reliability of new measures of cost-related medication nonadherence. Med Care. 2008:444-448.

110. Maselli DJ, Hardin M, Christenson SA, et al. Clinical Approach to the Therapy of Asthma-COPD Overlap. Chest. 2019;155(1):168-177. doi:10.1016/j.chest.2018.07.028

111. Jena AB, Ho O, Goldman DP, Karaca-Mandic P. The impact of the US Food and Drug Administration chlorofluorocarbon ban on out-of-pocket costs and use of albuterol inhalers among individuals with asthma. JAMA Intern Med. 2015;175(7):1171-1179.

112. Patel MR, Press VG, Gerald LB, et al. Improving the affordability of prescription medications for people with chronic respiratory disease. An official American Thoracic Society policy statement. Am J Respir Crit Care Med. 2018;198(11):1367-1374.

113. George CJ, Jacobs LG. Geriatrics medication management rounds: a novel approach to teaching rational prescribing with the use of the medication screening questionnaire. $J \mathrm{Am}$ Geriatr Soc. 2011;59(1):138-142.

114. Wilson IB, Schoen C, Neuman P, et al. Physician-patient communication about prescription medication nonadherence: A 50-state study of America's seniors. J Gen Intern Med. 2007;22(1):6-12. doi:10.1007/s11606-006-0093-0

115. Appel LJ, Espeland MA, Easter L, Wilson AC, Folmar S, Lacy CR. Effects of reduced sodium intake on hypertension control in older individuals: results from the Trial of Nonpharmacologic Interventions in the Elderly (TONE). Arch Intern Med. 2001;161(5):685-693.

116. Whelton PK, Appel LJ, Espeland MA, et al. Sodium reduction and weight loss in the 
treatment of hypertension in older persons: a randomized controlled trial of nonpharmacologic interventions in the elderly (TONE). Jama. 1998;279(11):839-846.

117. Holmes HM, Min LC, Yee M, et al. Rationalizing prescribing for older patients with multimorbidity: considering time to benefit. Drugs Aging. 2013;30(9):655-666.

118. Axelsson M, Emilsson M, Brink E, Lundgren J, Torén K, Lötvall J. Personality, adherence, asthma control and health-related quality of life in young adult asthmatics. Respir Med. 2009;103(7):1033-1040. doi:https://doi.org/10.1016/j.rmed.2009.01.013

119. Marceau C, Lemière C, Berbiche D, Perreault S, Blais L. Persistence, adherence, and effectiveness of combination therapy among adult patients with asthma. J Allergy Clin Imтипоl. 2006;118(3):574-581. doi:10.1016/j.jaci.2006.06.034

120. Stoloff SW, Stempel DA, Meyer J, Stanford RH, Rosenzweig JRC. Improved refill persistence with fluticasone propionate and salmeterol in a single inhaler compared with other controller therapies. J Allergy Clin Immunol. 2004;113(2):245-251.

121. Stempel DA, Stoloff SW, Rosenzweig JRC, Stanford RH, Ryskina KL, Legorreta AP. Adherence to asthma controller medication regimens. Respir Med.2005;99(10):12631267.

122. Ringdal N, Eliraz A, Pruzinec P, et al. The salmeterol/fluticasone combination is more effective than fluticasone plus oral montelukast in asthma. Respir Med. 2003;97(3):234241.

123. O'BYRNE PM, Barnes PJ, Rodriguez-Roisin R, et al. Low dose inhaled budesonide and formoterol in mild persistent asthma: the OPTIMA randomized trial. Am J Respir Crit Care Med. 2001;164(8):1392-1397.

124. Corsonello A, Pedone C, Lattanzio F, et al. Regimen complexity and medication nonadherence in elderly patients. Ther Clin Risk Manag. 2009;5(1):209-216. doi: $10.2147 /$ tcrm.s4870

125. Walsh CA, Cahir C, Tecklenborg S, Byrne C, Culbertson MA, Bennett KE. The association between medication non-adherence and adverse health outcomes in ageing populations: A systematic review and meta-analysis. Br J Clin Pharmacol. 2019;85(11):2464-2478.

126. van Boven JFM, Chavannes NH, van der Molen T, Rutten-van Mölken MPMH, Postma MJ, Vegter S. Clinical and economic impact of non-adherence in COPD: a systematic review. Respir Med. 2014;108(1):103-113.

127. Williams LK, Pladevall M, Xi H, et al. Relationship between adherence to inhaled corticosteroids and poor outcomes among adults with asthma. J Allergy Clin Immunol. 
2004;114(6):1288-1293. doi:10.1016/j.jaci.2004.09.028

128. Suissa S, Ernst P, Benayoun S, Baltzan M, Cai B. Low-dose inhaled corticosteroids and the prevention of death from asthma. N Engl J Med. 2000;343(5):332-336.

129. Li Y, Zhou H, Cai B, et al. Group-based trajectory modeling to assess adherence to biologics among patients with psoriasis. Clinicoecon Outcomes Res. 2014;6:197-208. doi:10.2147/CEOR.S59339

130. MacEwan JP, Forma FM, Shafrin J, Hatch A, Lakdawalla DN, Lindenmayer J-P. Patterns of adherence to oral atypical antipsychotics among patients diagnosed with schizophrenia. J Manag care Spec Pharm. 2016;22(11):1349-1361.

131. Nagin DS, Odgers CL. Group-Based Trajectory Modeling in Clinical Research. Annu Rev Clin Psychol. 2010;6(1):109-138. doi:10.1146/annurev.clinpsy.121208.131413

132. Hoch H, Pickett K, Brinton J, Szefler SJ. Evaluating Adherence Data in the Clinical Setting: What Tools Should We Use to Describe Behavior? In: American Thoracic Society International Conference Meetings Abstracts American Thoracic Society International Conference Meetings Abstracts. American Thoracic Society; 2019:A6735-A6735. doi:10.1164/ajrccm-conference.2019.199.1_meetingabstracts.a6735

133. van Boven JFM, Koponen M, Lalic S, et al. Trajectory Analyses of Adherence Patterns in a Real-Life Moderate to Severe Asthma Population. J Allergy Clin Immunol Pract. December 2020. doi:10.1016/j.jaip.2019.12.002

134. Franklin JM, Shrank WH, Pakes J, et al. Group-based Trajectory Models: A New Approach to Classifying and Predicting Long-Term Medication Adherence. Med Care. 2013;51:789-796. doi:10.2307/42568759

135. Nagin DS, NAGIN D. Group-Based Modeling of Development. Harvard University Press; 2005.

136. Nagin DS. Analyzing developmental trajectories: a semiparametric, group-based approach. Psychol Methods. 1999;4(2):139.

137. Jones BL, Nagin DS. Proc TRAJ: A SAS Procedure for Group-Based Modeling of Longitudinal Data. In: Annual Meeting Home. ; 2007.

138. Benzo R, Vickers K, Ernst D, Tucker S, McEvoy C, Lorig K. Development and feasibility of a self-management intervention for chronic obstructive pulmonary disease delivered with motivational interviewing strategies. J Cardiopulm Rehabil Prev. 2013;33(2):113123.

139. Benzo R, Vickers K, Novotny PJ, et al. Health coaching and chronic obstructive pulmonary disease rehospitalization. A randomized study. Am J Respir Crit Care Med. 
2016;194(6):672-680.

140. Wilson SR, Strub P, Buist AS, et al. Shared treatment decision making improves adherence and outcomes in poorly controlled asthma. Am J Respir Crit Care Med. 2010;181(6):566-577.

141. Cecere LM, Slatore CG, Uman JE, et al. Adherence to long-acting inhaled therapies among patients with chronic obstructive pulmonary disease (COPD). COPD J Chronic Obstr Pulm Dis. 2012;9(3):251-258.

142. Perrin K, Williams M, Wijesinghe M, James K, Weatherall M, Beasley R. Randomized controlled trial of adherence with single or combination inhaled corticosteroid/long-acting $\beta$-agonist inhaler therapy in asthma. J Allergy Clin Immunol. 2010;126(3):505-510.

143. Matsunaga K, Kawabata H, Hirano T, Sugiura H, Minakata Y, Ichinose M. Difference in time-course of improvement in asthma control measures between budesonide and budesonide/formoterol. Pulm Pharmacol Ther. 2013;26(2):189-194.

144. Restrepo RD, Alvarez MT, Wittnebel LD, et al. Medication adherence issues in patients treated for COPD. Int J Chron Obstruct Pulmon Dis. 2008;3(3):371. 


\section{Appendices}

\section{Appendix 7.1}

Attrition Table of Individuals Included in the Analysis Based on Inclusion and Exclusion Criteria

Number of individuals available in MCBS 2006-2013

\section{0,551}

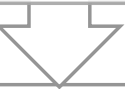

Number of individuls at least 65 yeas old

74,689

\begin{tabular}{|c|}
\hline Number of individuals with at least one full year enrollment \\
\hline $\mathbf{6 8 , 3 5 2}$ \\
\hline
\end{tabular}

Number of individuls with community-dwelling status

\section{1,819}

\begin{tabular}{|c|}
\hline Number of individuals who were alive during calendar year \\
\hline $\mathbf{3 9 , 4 4 6}$ \\
\hline Number of individuls without ghost id \\
\hline $\mathbf{3 9 , 2 9 5}$ \\
\hline Number of individuls who are enrolled in fee-for-service program \\
\hline $\mathbf{3 8 , 0 8 9}$
\end{tabular}


Appendix 7.2

Diagnosis Codes for Asthma and COPD

\begin{tabular}{|c|c|c|}
\hline & ICD-9-CM & ICD-10-CM \\
\hline 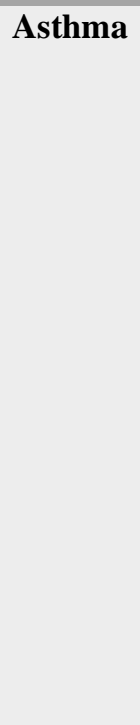 & $\begin{array}{l}493.0 \\
493.1 \\
493.2 \\
493.8 \\
493.9\end{array}$ & $\begin{array}{l}\mathrm{J} 45.20 \\
\mathrm{~J} 45.21 \\
\mathrm{~J} 45.22 \\
\mathrm{~J} 45.30 \\
\mathrm{~J} 45.31 \\
\mathrm{~J} 45.32 \\
\mathrm{~J} 45.40 \\
\mathrm{~J} 45.41 \\
\mathrm{~J} 45.42 \\
\mathrm{~J} 45.50 \\
\mathrm{~J} 45.51 \\
\mathrm{~J} 45.52 \\
\mathrm{~J} 45.901 \\
\mathrm{~J} 45.902 \\
\mathrm{~J} 45.909 \\
\mathrm{~J} 45.990 \\
\mathrm{~J} 45.991 \\
\mathrm{~J} 45.998\end{array}$ \\
\hline COPD & $\begin{array}{l}491.1 \\
491.2 \\
491.8 \\
491.9 \\
492 \\
492.8 \\
496\end{array}$ & $\begin{array}{l}\mathrm{J} 41.0 \\
\mathrm{~J} 41.1 \\
\mathrm{~J} 41.8 \\
\mathrm{~J} 42 \\
\mathrm{~J} 43.0 \\
\mathrm{~J} 43.1 \\
\mathrm{~J} 43.2 \\
\mathrm{~J} 43.8 \\
\mathrm{~J} 43.9 \\
\mathrm{~J} 44.0 \\
\mathrm{~J} 44.1 \\
\mathrm{~J} 44.9\end{array}$ \\
\hline
\end{tabular}


Appendix 7.3

Characteristics of Elderly Community-Dwelling Fee-for-Service Medicare Beneficiaries

\begin{tabular}{|c|c|c|}
\hline & $\mathbf{N}$ & Wt. \% \\
\hline ALL & 38,089 & 100.0 \\
\hline \multicolumn{3}{|l|}{ Sex } \\
\hline Female & 21,469 & 56.2 \\
\hline Male & 16,620 & 43.8 \\
\hline \multicolumn{3}{|l|}{ Age in Years } \\
\hline $65-74$ & 15,107 & 47.5 \\
\hline $75-84$ & 15,648 & 37.5 \\
\hline 85 and above & 7,334 & 15.1 \\
\hline \multicolumn{3}{|l|}{ Race/Ethnicity } \\
\hline White & 31,529 & 82.6 \\
\hline Black & 2,603 & 6.7 \\
\hline Latino & 2,145 & 5.7 \\
\hline Other race & 1,733 & 4.8 \\
\hline \multicolumn{3}{|l|}{ Marital Status } \\
\hline Married & 19,775 & 54.2 \\
\hline Not married & 18,298 & 45.7 \\
\hline \multicolumn{3}{|l|}{ Education } \\
\hline Less than high school & 9,256 & 22.2 \\
\hline High school & 13,751 & 35.5 \\
\hline Above high school & 14,935 & 41.9 \\
\hline \multicolumn{3}{|l|}{ Poverty Status } \\
\hline Less than $200 \%$ FPL & 18,382 & 45.6 \\
\hline At least 200\% FPL & 19,707 & 54.4 \\
\hline \multicolumn{3}{|l|}{ Prescription Coverage } \\
\hline Yes & 23,700 & 61.6 \\
\hline No & 14,389 & 38.4 \\
\hline \multicolumn{3}{|c|}{ Number of Unique Medications } \\
\hline 0 & 1,714 & 5.1 \\
\hline $1-4$ & 9,266 & 25.3 \\
\hline $5-7$ & 9,008 & 23.5 \\
\hline $8-11$ & 8,897 & 22.8 \\
\hline 12 and more & 9,204 & 23.4 \\
\hline \multicolumn{3}{|l|}{ Number of Other Chronic } \\
\hline \multicolumn{3}{|l|}{ Conditions } \\
\hline $0-1$ & 7,845 & 22.3 \\
\hline $2-3$ & 20,286 & 53.0 \\
\hline $4-6$ & 9,949 & 24.6 \\
\hline \multicolumn{3}{|l|}{ Calendar Year } \\
\hline 2006 & 5,776 & 13.2 \\
\hline 2007 & 5,382 & 12.3 \\
\hline 2008 & 4,965 & 11.7 \\
\hline 2009 & 4,341 & 11.5 \\
\hline 2010 & 4,077 & 11.5 \\
\hline 2011 & 4,634 & 13.4 \\
\hline 2012 & 4,602 & 13.2 \\
\hline 2013 & 4,312 & 13.3 \\
\hline
\end{tabular}

Note: Based on 38,089 person-years of community-dwelling elderly (age $\geq 65$ years) Medicare beneficiaries continuously enrolled in fee-for-service Part A and Part B during the calendar year, alive during the calendar year.

FPL: federal poverty level; N: unweighted number; Wt: weighted. 
Appendix 7.4.

Mean Annual Adjusted and Unadjusted Expenditure

Among Older (age > 65 years) Medicare Beneficiaries

Medicare Current Beneficiary Survey, 2006-2013

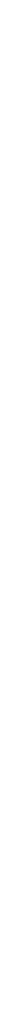

Note: Based on 38,089 person-years of community-dwelling elderly (age $\geq 65$ years) Medicare beneficiaries, continuously enrolled in fee-for-service Part A and Part B during the calendar year and alive during the calendar year. Incremental costs were measured using counterfactual recycled predictions performed using estimates obtained from a 2-part GLM model with log link and gamma distribution. The variables in the adjusted models were selected based on Andersen's healthcare behavioral model and include sex, age, race, marital status, poverty, education, prescription coverage, fragmented care, \# ADL, \# chronic conditions, \# unique medications, overweight, smoking habit, and residency region.

COPD: Chronic Obstructive Pulmonary Disease; CRN: Cost-related Medication Non-Adherence; Ref: reference group; Sig: significance***p<.001; ${ }^{* *} .001 \leq \mathrm{p}<.01 ; * .01 \leq \mathrm{p}<.05$ 


\begin{tabular}{|c|c|c|c|c|c|c|}
\hline \multicolumn{7}{|c|}{$\begin{array}{c}\text { Appendix } 7.5 \\
\text { Unadjusted Odds Ratios (UOR), Adjusted Odds Ratios (AOR) and 95\% Confidence Intervals from } \\
\text { Separate Logistic Regressions on High Out-of-Pocket Burden } \\
\text { Older (age } \geq 65 \text { years) Medicare Beneficiaries } \\
\text { Medicare Current Beneficiary Survey, 2006-2013 }\end{array}$} \\
\hline & \multicolumn{3}{|c|}{ Unadjusted Model } & \multicolumn{3}{|c|}{ Fully Adjusted Model } \\
\hline & UOR & $95 \% \mathrm{CI}$ & Sig & AOR & $95 \% \mathrm{CI}$ & Sig \\
\hline \multicolumn{7}{|l|}{ Asthma COPD Status } \\
\hline Asthma COPD Overlap & 3.51 & {$[2.96,4.16]$} & & 1.31 & {$[1.07,1.61]$} & $* *$ \\
\hline Asthma Only & 1.99 & {$[1.68,2.36]$} & & 1.19 & {$[0.99,1.42]$} & \\
\hline $\begin{array}{l}\text { COPD only } \\
\text { No asthma No COPD (ref) }\end{array}$ & 2.40 & {$[2.20,2.61]$} & & 1.28 & {$[1.16,1.42]$} & $* * *$ \\
\hline \multicolumn{7}{|l|}{ 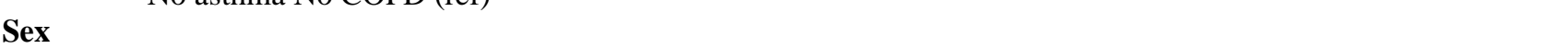 } \\
\hline $\begin{array}{l}\text { Female } \\
\text { Male (ref) }\end{array}$ & & & & 1.14 & {$[1.05,1.23]$} & $* *$ \\
\hline Age in Years & & & & 1.02 & {$[1.01,1.02]$} & \\
\hline \multicolumn{7}{|l|}{$\begin{array}{l}\text { Race/Ethnicity } \\
\text { White (ref) }\end{array}$} \\
\hline Black & & & & 0.82 & {$[0.72,0.94]$} & $* *$ \\
\hline Latino & & & & 0.68 & {$[0.56,0.83]$} & $* * *$ \\
\hline Other Races & & & & 0.60 & {$[0.50,0.72]$} & $* * *$ \\
\hline \multicolumn{7}{|l|}{ Marital Status } \\
\hline $\begin{array}{l}\text { Married } \\
\text { Not married (ref) }\end{array}$ & & & & 0.69 & {$[0.64,0.74]$} & $* * *$ \\
\hline \multicolumn{7}{|l|}{ Education } \\
\hline \multicolumn{7}{|l|}{ Less than high school (ref) } \\
\hline High school & & & & 0.77 & {$[0.68,0.86]$} & $* * *$ \\
\hline Above high school & & & & 0.93 & {$[0.84,1.03]$} & \\
\hline \multicolumn{7}{|l|}{ Poverty Status } \\
\hline $\begin{array}{l}\text { Less than } 200 \% \text { FPL } \\
\text { At least } 200 \% \text { FPL (ref) }\end{array}$ & & & & 3.71 & {$[3.44,4.00]$} & $* * *$ \\
\hline \multicolumn{7}{|l|}{ Prescription Coverage } \\
\hline $\begin{array}{l}\text { Yes } \\
\text { No (ref) }\end{array}$ & & & & 0.56 & {$[0.52,0.60]$} & $* * *$ \\
\hline \% Fragmented Care & & & & 1.01 & {$[1.01,1.01]$} & *** \\
\hline \# Other Chronic Conditions & & & & 1.05 & {$[1.02,1.09]$} & $* * *$ \\
\hline \# ADL & & & & 1.12 & {$[1.09,1.15]$} & $* * *$ \\
\hline \# Unique Medications & & & & 1.09 & {$[1.08,1.10]$} & $* * *$ \\
\hline \multicolumn{7}{|l|}{ Overweight } \\
\hline $\begin{array}{l}\text { Yes } \\
\text { No (ref) }\end{array}$ & & & & 1.01 & {$[0.94,1.10]$} & \\
\hline \multicolumn{7}{|l|}{ Smoking habit } \\
\hline $\begin{array}{l}\text { Current and Past } \\
\text { Never (ref) }\end{array}$ & & & & 1.14 & {$[0.93,1.34]$} & \\
\hline \multicolumn{7}{|l|}{ Residency Region } \\
\hline Northeast & & & & 0.95 & {$[0.81,1.11]$} & \\
\hline South & & & & 0.79 & {$[0.71,0.87]$} & *** \\
\hline $\begin{array}{l}\text { Midwest } \\
\text { West (ref) }\end{array}$ & & & & 0.90 & {$[0.80,1.01]$} & \\
\hline
\end{tabular}




\section{Appendix 7.6 \\ Post-Regression Decomposition Analyses \\ Older (age $\geq 65$ years) Medicare Beneficiaries \\ Medicare Current Beneficiary Survey, 2006-2013}

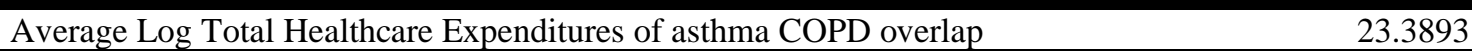

$\begin{array}{lc}\text { Average Log Total Expenditures of asthma only group } & 20.1912\end{array}$

$\begin{array}{lc}\text { Difference in Log Total Healthcare Expenditures } & 11.1627\end{array}$

Total "explained" percentage

\section{$\%$ Contribution to the "Explained" Portion}

Predisposing characteristics (sex, age, race)

76.42

Enabling factors 1 (marital status, poverty, education, prescription coverage)

$3.70 \%$

Enabling factor 2 (fragmented care)

Need Factors 1 (\# ADL, \# chronic conditions)

$11.48 \% *$

Need Factors 2 (\# unique medications)

$42.05 \%$

Personal healthcare practice (obesity, smoking)

$76.73 \% *$

External factors (residency region)

$-21.12 \%$

Average Log Total Healthcare Expenditures of asthma COPD overlap

$-9.08 \%$

Average Log Total Expenditures of COPD only group

20.8304

20.0288

Difference in Log Total Healthcare Expenditures

0.080157

Total "explained" percentage

\section{$\%$ Contribution to the "Explained" Portion}

Predisposing characteristics (sex, age, race)

$45.12 \%$

Enabling factors 1 (marital status, poverty, education, prescription coverage)

Enabling factor 2 (fragmented care)

$-109.10 \%$

$32.61 \%$

$29.12 \%$

Need Factors 1 (\# ADL, \# chronic conditions)

$28.19 \%$

Need Factors 2 (\# unique medications)

Personal healthcare practice (obesity, smoking)

$61.51 \% * * *$

External factors (residency region)

Notes: Based on 38,089 elderly Medicare beneficiaries who were observed between years 2006 and 2013. The percent differences of domains of Andersen's healthcare behavior model are illustrated in the model. 
Appendix 7.7

Attrition Table of Individuals Included in the Analysis Based on Inclusion and Exclusion Criteria

Number of individuals available in MCBS 2006-2013

$\mathbf{1 3 1 , 0 0 7}$

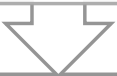

Number of individuls at least 65 yeas old

74,689

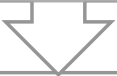

Number of individuals with at least one full year enrollment

$\mathbf{7 0 , 4 7 0}$

Number of individuls with community-dwelling status

68,512

$\checkmark$

Number of individuals who were alive during calendar year

63,923

Number of individuls who are enrolled in fee-for-service program

61,870

Number of individuals whose information was available in both "access to care" and "cost and use" files

37,817

Number of individuls wihout any missing values for cost-related medication non-adherence

37,571 
Appendix 7.8

Characteristics of Elderly Community-Dwelling Fee-for-Service Medicare Beneficiaries

\begin{tabular}{|c|c|c|}
\hline & $\mathbf{N}$ & Wt. \% \\
\hline ALL & 37,571 & 100.0 \\
\hline \multicolumn{3}{|l|}{ Sex } \\
\hline Female & 21,186 & 56.2 \\
\hline Male & 16,385 & 43.8 \\
\hline \multicolumn{3}{|l|}{ Age in Years } \\
\hline $65-74$ & 14,893 & 47.4 \\
\hline $75-84$ & 15,447 & 37.5 \\
\hline 85 and above & 7,231 & 15.1 \\
\hline \multicolumn{3}{|l|}{ Race/Ethnicity } \\
\hline White & 31,130 & 82.7 \\
\hline Black & 2,563 & 6.6 \\
\hline Latino & 2,112 & 5.7 \\
\hline Other race & 1,688 & 4.8 \\
\hline \multicolumn{3}{|l|}{ Marital Status } \\
\hline Married & 19,507 & 54.2 \\
\hline Not married & 18,050 & 45.7 \\
\hline \multicolumn{3}{|l|}{ Education } \\
\hline Less than high school & 9,145 & 22.2 \\
\hline High school & 13,573 & 35.5 \\
\hline Above high school & 14,711 & 41.9 \\
\hline \multicolumn{3}{|l|}{ Poverty Status } \\
\hline Less than $200 \%$ FPL & 18,120 & 45.6 \\
\hline At least $200 \%$ FPL & 19,451 & 54.4 \\
\hline \multicolumn{3}{|l|}{ Prescription Coverage } \\
\hline Yes & 23,380 & 61.6 \\
\hline No & 14,191 & 38.4 \\
\hline \multicolumn{3}{|l|}{ OOP High Burden-Prescription Costs } \\
\hline Yes & 1,805 & 4.5 \\
\hline No & 35,766 & 95.5 \\
\hline \multicolumn{3}{|l|}{ OOP High Burden - Medical Costs } \\
\hline Yes & 4,885 & 12.3 \\
\hline No & 32,686 & 87.7 \\
\hline \multicolumn{3}{|l|}{ Number of Unique Medications } \\
\hline 0 & 1,665 & 5.0 \\
\hline $1-4$ & 9,142 & 25.3 \\
\hline $5-7$ & 8,906 & 23.5 \\
\hline $8-11$ & 8,810 & 22.9 \\
\hline 12 and more & 9,048 & 23.3 \\
\hline \multicolumn{3}{|l|}{ Number of Other Chronic Conditions } \\
\hline $0-1$ & 7,687 & 22.2 \\
\hline $2-3$ & 20,037 & 53.1 \\
\hline $4-6$ & 9,842 & 24.7 \\
\hline \multicolumn{3}{|l|}{ Calendar Year } \\
\hline 2006 & 5,694 & 13.2 \\
\hline 2007 & 5,321 & 12.3 \\
\hline 2008 & 4,904 & 11.7 \\
\hline 2009 & 4,265 & 11.4 \\
\hline 2010 & 4,001 & 11.4 \\
\hline 2011 & 4,581 & 13.4 \\
\hline 2012 & 4,546 & 13.2 \\
\hline 2013 & 4,259 & 13.3 \\
\hline
\end{tabular}

Note: Based on 37,571 person-years of community-dwelling elderly (age $\geq 65$ years) Medicare beneficiaries continuously enrolled in fee-for-service Part A and Part B during the calendar year, alive during the calendar year, had information in both cost and use and access to care files and did not have missing information on any costrelated medication nonadherence measures.

FPL: federal poverty level; N: unweighted number; Wt: weighted. 
Appendix 7.9

Attrition Table of Individuals Included in the Analysis Based on Inclusion and Exclusion Criteria

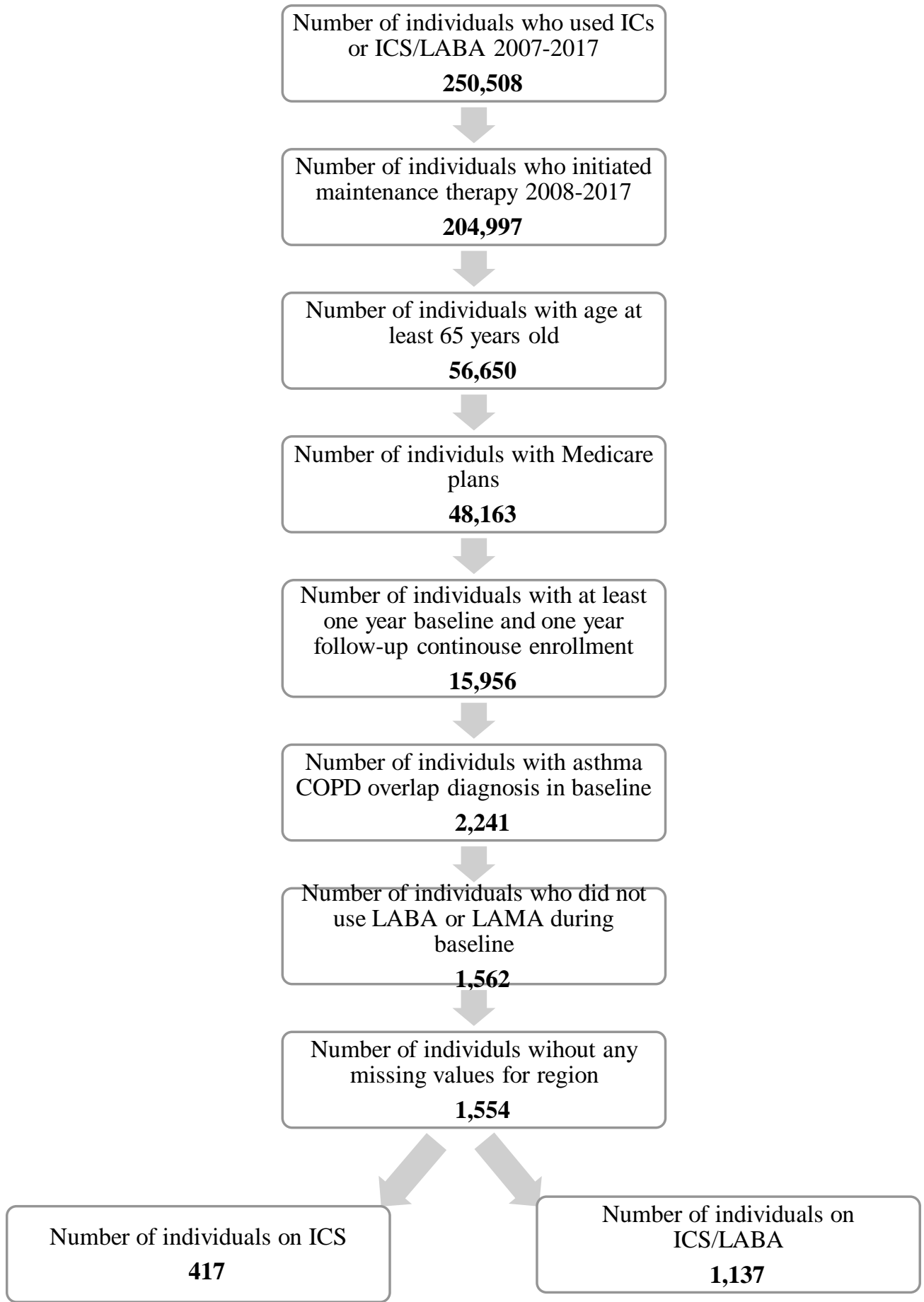


Appendix 7.10

Adjusted Odds Ratios (AOR) and 95\% Confidence Intervals from

Generalized Estimating Equation (GEE) on Adherence

among older adults with ACO Who Newly Initiated Maintenance Therapy

Optum Clinformatics 10\% Sample, 2008-2016

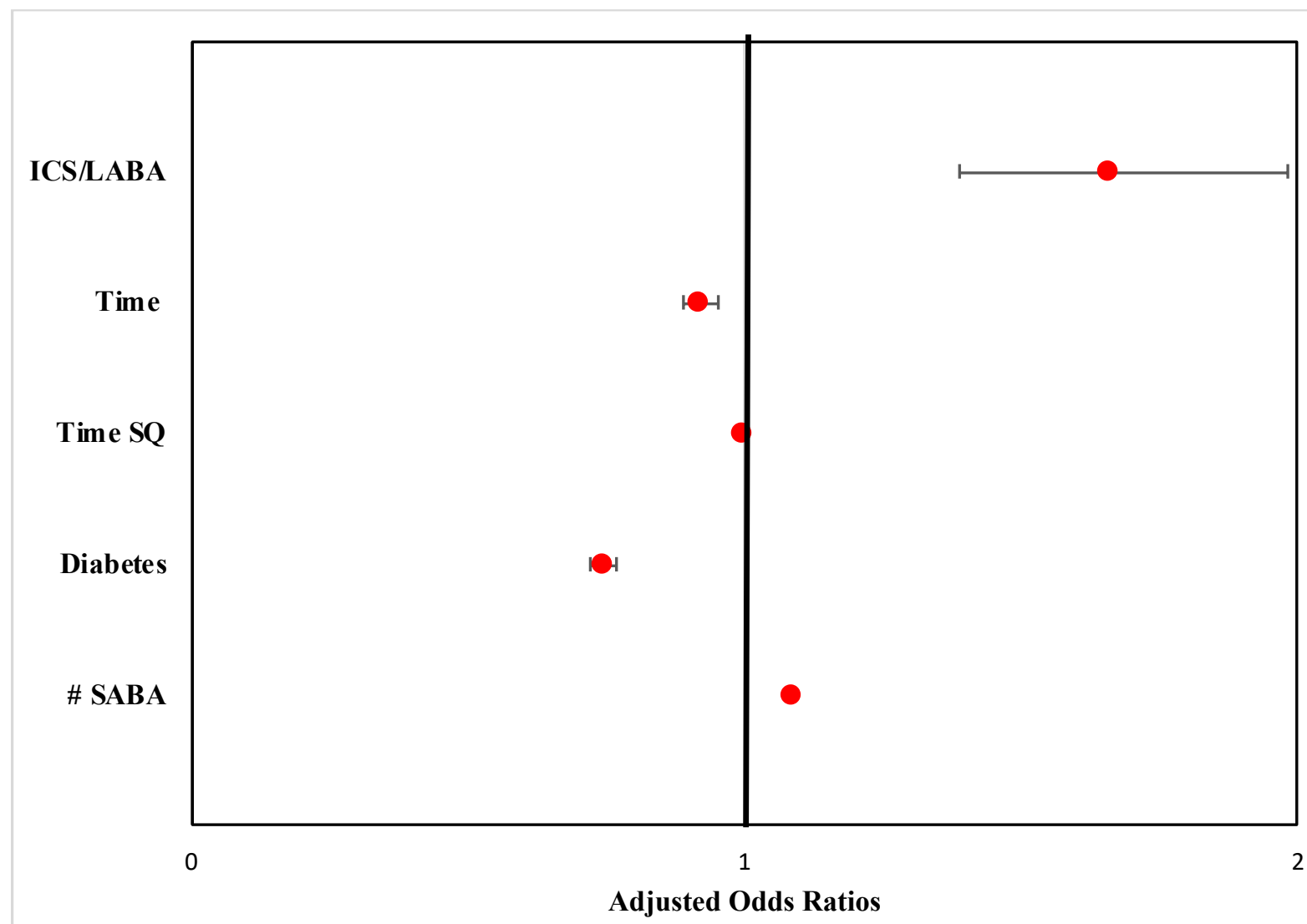

Note: Based on 1,556 Medicare beneficiaries (age > 65 years old) with ACO diagnosis and newly IMT (either ICS or ICS/LABA) who were continuously enrolled 24 months during the observation period. In the graph, we only illustrated the variables with significant association with adherence. The GEE model was adjusted by time, square time and all factors based on Andersen's model.

Abbreviations: ICS/LABA: Fixed dose combination of inhaled corticosteroid-and-long-acting beta agonist; OOP: Out of Pocket; ref: reference group; SABA: Short-Acting Beta Agonist; SQ: Square; \#: Number 


\section{Appendix 7.11}

Characteristics of Older Adults with Asthma-COPD Overlap

by Initial Maintenance Therapy Options

before Using Inverse Probability Treatment Weighting

Optum Clinformatics 10\% Sample, 2007-2017

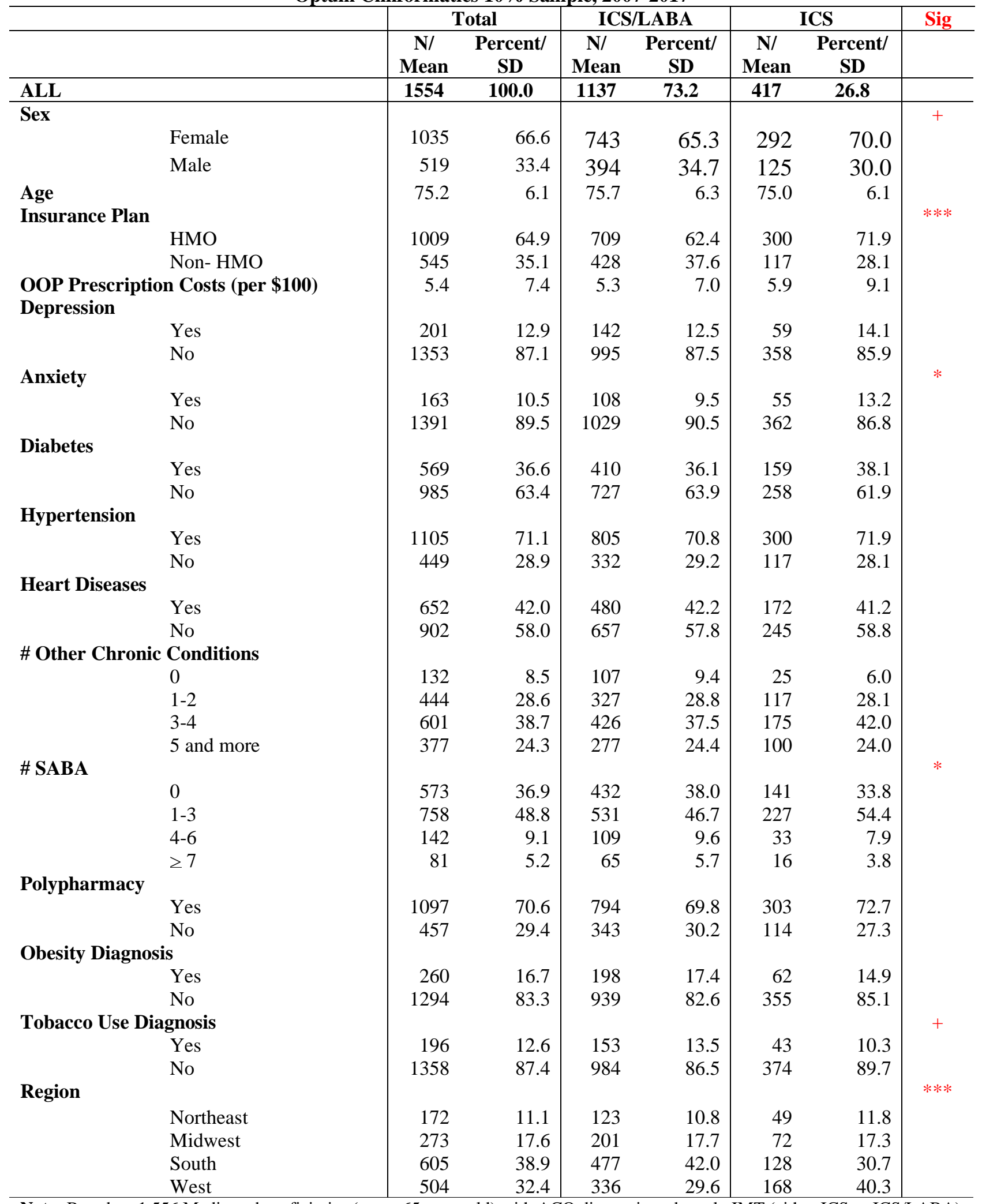

Note: Based on 1,556 Medicare beneficiaries (age > 65 years old) with ACO diagnosis and newly IMT (either ICS or ICS/LABA) v continuously enrolled 24 months during the observation period. 
Abbreviations: ACO: Asthma COPD Overlap; HMO: Health Maintenance Organization; ICS: Inhaled Corticosteroid;

ICS/LABA: Fixed dose combination of inhaled corticosteroid-and-long-acting beta agonist; IMT: Initial Maintenance Therapy; N: Number; OOP: Out of Pocket; SABA: Short-Acting Beta Agonist; SD: Standard deviation; Sig: Significance; \#: Number $* * * \mathrm{p}<.001, * * .001 \leq \mathrm{p}<.01, * .01 \leq \mathrm{p}<.005,+.05 \leq \mathrm{p}<.1$ 